\title{
Generalized spaces for constructive algebra
}

\author{
Ingo Blechschmidt \\ Università di Verona \\ Department of Computer Science \\ Strada le Grazie 15 \\ 37134 Verona, Italy
}

The purpose of this contribution is to give a coherent account of a particular narrative which links locales, geometric theories, sheaf semantics and constructive commutative algebra. We are hoping to convey a firm grasp of three ideas: (1) Locales are a kind of space in which opens instead of points are fundamental. (2) Sheaf semantics allows us to explore mathematical objects from custom-tailored mathematical universes. (3) Without loss of generality, any reduced ring is a field.

\section{Contents}

Generalized spaces for constructive algebra

Introduction . . . . . . . . . . . . . . . . . . . . . . .

1. Locales . . . . . . . . . . . . . . . . . . . . 7

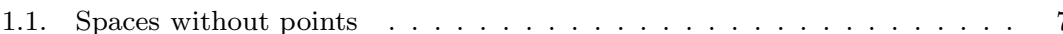

1.2. Constructive concerns . . . . . . . . . . . . . . . . 10

1.3. The basics of the theory of locales . . . . . . . . . . . . . . . . 13

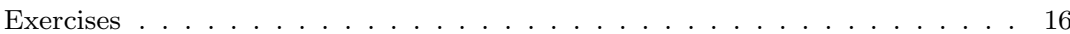

2. Geometric theories and sheaf models . . . . . . . . . . . . . . . . . . . 19

2.1. Geometric theories . . . . . . . . . . . . . . . . . . . . . . 19

2.2. Presenting frames by theories . . . . . . . . . . . . . . . . 29

2.3. Sheaves on locales . . . . . . . . . . . . . . . . . . . . . . . . . . . . . . . . . . . . . . . . . .

2.4. Sheaf semantics ...................... . . . . . . . . . . . . . . . . . . . . . . .

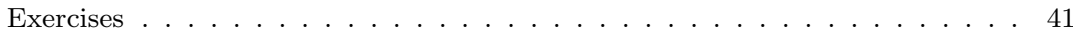

3. Applications in constructive algebra . . . . . . . . . . . . . . . . . . . . . . . . . . . . . . . . . . .

3.1. Algebraic preliminaries . . . . . . . . . . . . . . . . . 46

3.2. A remarkable sheaf ..................... . . . . . . . . . . . . . . . . . . . . . . .

3.3. An algebraic origin story . . . . . . . . . . . . . . . . . . . . . . . . . . . . . . . . . . . . . . .

3.4. Understanding the sheaf model . . . . . . . . . . . . . . . . 57

3.5. Evaluating test cases . . . . . . . . . . . . . . . 63 


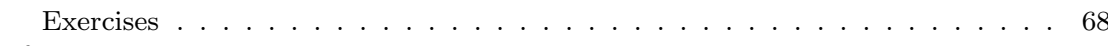

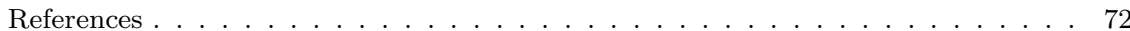

\section{Introduction a}

Locales. The notion of space is fundamental to large parts of mathematics. This notion exists in various flavors, ranging from the basic cartesian spaces $\mathbb{R}^{n}$ to the more general metric and topological spaces and also including several more and slightly exotic flavors such as the diffeological spaces from differential topology.

Common to all of the mentioned flavors of space is that points are their building blocks: cartesian, metric, topological and diffeological spaces are, first and foremost, sets of points. Their spatial structure - a metric, a topology or a diffeology - is additional data to the underlying set:

Definition. A metric space consists of a set $X$ of points together with a map $d: X \times X \rightarrow \mathbb{R}_{\geq 0}$ satisfying the metric axioms.

Definition. A topological space consists of a set $X$ of points together with a set $\mathcal{O}(X) \subseteq P(X)$ of point sets which are deemed open such that (arbitrary, set-indexed) unions and finite intersections of open sets are open.

Locales are a further and particularly unique flavor of the notion of space which turn this classical picture upside down: Locales embrace opens instead of points as primitive building blocks. With locales, points are a derived concept. In particular, the opens of a locale are not sets of points; in fact, they need not be sets of anything in peculiar.

Section 1 explores the basics of the theory of locales, with a focus on examples and issues for constructive mathematics. Accessible introductions to the theory include Refs. 3, 4. The interplay between logic and geometry is particularly stressed in Refs. [5, 6]. We recommend Ref. [7] for a historical guide and Ref. [8] for a comprehensive modern textbook on the subject.

\footnotetext{
${ }^{\mathrm{a} U n l i k e}$ some other texts on locales or sheaves, this text is set in a constructive (but impredicative) metatheory. We do not use the law of excluded middle nor any version of the axiom of choice, and we also do not adopt any nonclassical principles. We freely use powersets, but more for linguistic convenience than by necessity. In particular, the central idea presented in Section 3 the reduction technique by which we can pretend that any given reduced ring is a field, is very robust with respect to the metatheory. Even though we chose a semantic point of view for its derivation, the final result is a purely syntactical transformation. As it stands, this text could be formalized in the kind of type theory which is valid in toposes [1] or in IZF [2].
} 
Geometric theories and sheaf models. The most sensible solution to the task

$$
\begin{aligned}
& \text { Determine the continuous complex-valued functions } f \\
& \text { with } f(z)^{2}=z \text {. }
\end{aligned}
$$

is not the single set

$$
\left\{f: \mathbb{C} \rightarrow \mathbb{C} \mid f \text { is continuous and } f(z)^{2}=z \text { for all } z \in \mathbb{C}\right\} .
$$

Rather, since the solvability of this functional equation varies with the domain on which $f$ should be defined (having no global solution defined on all of $\mathbb{C}$ but two solutions on small disks not containing the origin), we should consider the family

$$
\left(\left\{f: U \rightarrow \mathbb{C} \mid f \text { is continuous and } f(z)^{2}=z \text { for all } z \in \mathbb{C}\right\}\right)_{U \in \mathcal{O}(\mathbb{C})}
$$

of sets as the solution (or perhaps, since no solution was actually given, as an apt description) of the task.

If we are building a prime ideal $\mathfrak{p}$ step by step, adding elements to it when the need arises, the most sensible answer to the question

$$
\text { Is } x \text { contained in the prime ideal } \mathfrak{p} \text { ? }
$$

is not a single truth value, but a family of truth values, parametrized by the possible stages of construction of $\mathfrak{p}$.

Somewhat surprisingly, these two examples can be treated by a single theory, the theory of sheaves. Sheaves help to organize such families in an efficient manner and reify them as single coherent entities; the first family is (part of) a sheaf of sets and the second family is (part of) a sheaf of truth values. Sheaves will be the subject of Sections 2.3 2.4.

Sheaves interact particularly well with geometric theories, which we review in Section 2.1. Firstly, sheaves allow us to generalize our notion of a model of a geometric theory. For instance, a set-based model of the geometric theory of rings is just an ordinary ring; but now we can also consider sheaves of rings as further models.

Some geometric theories only really unfold in the world of sheaves. For instance, the geometric theory of surjections $\mathbb{N} \rightarrow \mathbb{R}$ reviewed on page 22 does not have any set-based models, but this theory is consistent and it does have nontrivial sheaf models. For the purposes of constructive algebra, sheaf models of some geometric theories play a vital role, as we will discuss in Section 3 .

Secondly, geometric theories allow us to efficiently construct the spaces (locales) on which we want to study sheaves. This is because a fundamental feature of geometric theories (in contrast to, say, arbitrary first-order 
theories) is that their models naturally organize to form a space, the space (locale) of models reviewed in Section 2.2.

Applications in constructive algebra. Commutative algebra abounds with techniques to reduce given situations to simpler ones, for instance passing to a quotient or passing to a localization. These techniques facilitate short and elegant proofs, such as the following. (Section 3.1 contains some algebraic preliminaries.)

Theorem. Let $M$ be an injective matrix with more columns than rows over a reduced ring $A$. Then $1=0$ in $A$.

Proof. Assume not. Then there is a minimal prime ideal $\mathfrak{p} \subseteq A$. Since localization is exact, the matrix $M$ is also injective when considered as a matrix over the stalk $A_{\mathfrak{p}}$. Since $A_{\mathfrak{p}}$ is a field, this is a contradiction to basic linear algebra.

However, these reduction techniques typically require transfinite methods. The displayed proof appeals to the transfinite four times:

(1) The proof proceeds by contradiction, hence requires the law of excluded middle.

(2) The proof then requires a minimal prime ideal. Such an ideal can be obtained in two steps: First, the Boolean Prime Ideal Theorem BPIT is invoked to obtain some prime ideal. Then Zorn's lemma fabricates a minimal one $b$

(3) Finally, the proof exploits that the stalks of reduced rings at minimal prime ideals are fields. This requires two further invocations of BPIT $\mathrm{c}$

This state of affairs is not satisfactory. A statement as simple as the displayed example should admit an explicit calculational proof, presenting a concrete method for transforming the given conditional equations expressing injectivity into the equation $1=0$ without any appeals to the transfinite.

\footnotetext{
bStandard textbooks prove the statement "any nontrivial ring has a prime ideal" by using Zorn's lemma, which in the presence of the law of excluded middle is equivalent to the full axiom of choice; however, the statement is actually equivalent to the weaker Boolean Prime Ideal Theorem [9] 10]. The combined statement "any nontrivial ring has a minimal prime ideal" is equivalent to the full axiom of choice 11].

${ }^{\mathrm{c}}$ By BPIT, the intersection of all prime ideals of $A_{\mathfrak{p}}$ is its nilradical. Since $A_{\mathfrak{p}}$ is reduced, the nilradical is the zero ideal. Since the prime ideals of $A_{\mathfrak{p}}$ are in bijection with those prime ideals of $A$ which are contained in $\mathfrak{p}$, the ring $A_{\mathfrak{p}}$ has exactly one prime ideal. By BPIT, any noninvertible element of $A_{\mathfrak{p}}$ is contained in some prime ideal, hence in the zero ideal. Thus $A_{\mathfrak{p}}$ is a field.
} 
The completeness theorem for coherent logic [12, Corollary D1.5.10] even gives an a priori reason why (for a given matrix size) a finitary proof has to exist. However, since the proof of the completeness theorem itself uses BPIT (and indeed is equivalent to it), it does not give any indication how such a proof could be found. (Still, beautiful constructive proofs are known and presented, for instance, in a celebrated short note by Fred Richman on nontrivial uses of trivial rings [13] and in the recent textbook [14] by Henri Lombardi and Claude Quitté on constructive commutative algebra.)

The key issue with the usual reduction techniques in commutative algebra is their dependence on ideal objects such as prime or maximal ideals. In general, those objects can only be obtained by transfinite methods. However, in practice many arguments do not actually require completed ideal objects: Their computational core applies just as well to finite approximations of these ideal objects.

It is then a challenging task in mathematical logic to devise efficient means to extract this obscured constructive content in a mostly mechanical manner. We envision to reinterpret any given classical proof employing ideal objects in a constructive fashion, realizing ideal objects as convenient fictions.

Locales and sheaf models contribute to this program by providing new reduction techniques for commutative algebra. These techniques have a similar effect as the classical reduction techniques, but are powered by purely constructive underpinnings. In a nutshell, the idea is as follows.

(1) Instead of replacing a given ring with another (for instance a ring $A$ with one of its stalks $A_{\mathfrak{p}}$ at a minimal prime ideal), we replace a given ring with a sheaf of rings.

(2) We then maintain the convenient illusion that we are working with a plain old ring instead of a sheaf by employing the sheaf semantics.

In some cases, these new techniques go even beyond their classical role models. For instance, while a module need not be free "on a dense open" if all its stalks at minimal prime ideals are, a module $M$ is free in that sense if its mirror image $M^{\sim}$ is not not free. This observation is the basis for a new proof of Grothendieck's generic freeness lemma, reviewed in Section 3.5. which is shorter and arguably more perspicuous than the previously known classical proofs. 
Where to go from here. The themes which this contribution touches on are embedded into a larger context, and we invite readers to follow those interesting tangents.

Regarding spaces, the notion of locales can be modified in several ways. Firstly, there are sites and closely related toposes. [15, 16. Given two opens $U$ and $V$ of a locale, there is only a truth value as to whether $U$ is contained in $V$; with sites and toposes, there can be a nontrivial set of ways that $U$ is contained in $V$. In an orthogonal direction, there is the notion of a formal topology [17, 18. These implement the idea of pointfree topology in predicative settings. In this context also basics of algebraic geometry have been developed [19]21.

Regarding sheaf semantics, Section 2.4only presents the first-order case, but the theory can be extended to include unbounded quantification 22, function types and power types and even a fully-fledged type theory [1. The sheaf semantics of appropriate toposes has been used to give synthethic accounts of several fields, such as differential geometry [23], domain theory [24] and computability theory [25].

Regarding constructive algebra, there are by now several textbooks 14 , 26, 27. on constructive algebra available and there is a vast literature on the intriguing subject of constructivizing classical commutative algebra. Starting points include Refs. 28 31 .

Notes for readers familiar with the themes of this contribution. We present a self-contained proof of the simple version of Barr's theorem as Theorem [11 For reducing intuitionistic provability to geometric provability, we employ (what amounts to) the syntactic site, similar to the refined completeness result by Thierry Coquand [32] but closer to the standard description of the syntactic site. For reducing classical provability to intuitionistic provability, we employ a variant of the double negation translation. We were not able to track down a reference to this approach in the context of infinitary logic, but the basic idea has long been known and is summarized, for instance, in Ref. 33.

In Section 2.3. where we introduce sheaves, we should probably also introduce sites. They would fit the narrative well and allow for the construction of generic models of arbitrary geometric theories and not only propositional ones. They would also allow us to cut down on the required metatheory, sitting comfortably within predicative environments such as arithmetic universes [34, 35] or CZF. We do not, however, mainly to not require prerequisites in category theory. 
The technique presented in Section 3 can be regarded as a first-order (and, if desired, higher-order) coating of an underlying geometric theory. We propose to take this coating seriously; in particular to make good use of surprising nongeometric sequents validated by the generic model; and, for ease of use by algebraists, to cast it in semantic terms, even though it is all syntax behind the scenes d

\section{Locales}

The formal definition of a locale will be given below as Definition 4, but we first review examples and comment on the relevance of locales to constructive mathematics.

\subsection{Spaces without points}

A metric or topological space without any points is not very interesting: It is empty, and up to isomorphism there is only one such space. In contrast, a locale can be nontrivial even if it does not contain any points. This phenomenon is an instance of a general guiding principle, namely that relinquishing points increases flexibility.

The locale of surjections $\mathbb{N} \rightarrow \mathbb{R}$. As is well-known, there are no surjections from $\mathbb{N}$ to $\mathbb{R} \mathrm{E}$ Hence there is no interesting topological space

${ }_{\mathrm{d}}^{\mathrm{By}}$ now it is an established idea in constructive algebra that we should consider the theory of prime filters instead of the prime filters themselves, hoping that, for instance, a given mathematical proof of the statement "no prime filter contains $x$ " can also be cast in the geometric language of the theory, which then yields the nilpotency of $x$ constructively. If the given proof uses only geometric reasoning, this is immediate; if it uses intuitionistic reasoning, it can be compiled down (for instance precisely by the technique we use); and if it uses classical reasoning, a version of Barr's theorem might be applicable. The elusive actual prime filters of the ring are replaced with the concrete and purely syntactical generic prime filter.

This approach misses that the generic model is interesting on its own and in particular that it satisfies peculiar nongeometric sequents such as "nonunits are nilpotent" and "any ideal is not not finitely generated". In this sense, the generic model is better than the actual models it aims to replace, and we believe that potential applications of this insight should be properly explored.

e More precisely, it is a theorem of classical mathematics that the reals are uncountable in the sense of admitting no surjection from the naturals. The situation is more subtle in constructive mathematics. Firstly, in the absence of countable choice, the reals bifurcate into several distinct flavors, hence one needs to state which flavor of the reals one is referring to. Secondly, all known proofs of the uncountability of the Cauchy and the Dedekind reals assume either the law of excluded middle or the axiom of countable choice. In the absence of either of these axioms, only the MacNeille reals are known to 
of those surjections. The set $\{f: \mathbb{N} \rightarrow \mathbb{R} \mid f$ is a surjection $\}$ is empty, and there is not much more to say about this state of affairs. However, there is a well-defined and nontrivial locale $X$ of those surjections.

The points of $X$ are in canonical one-to-one correspondence with the surjections $\mathbb{N} \rightarrow \mathbb{R}$, hence $X$ does not have any points. But this locale does have uncountably many basic opens $U_{n x}$, where $n$ ranges over the naturals and $x$ ranges over the reals. We picture $U_{n x}$ as the "open of those surjections $f$ for which $f(n)=x$ " and we can compute with these opens, consider functions on $X$ and ponder sublocales of $X$. For instance:

(1) If $x \neq y$, then the intersection of $U_{n x}$ with $U_{n y}$ is truly empty.

(2) The union of the $U_{n x}$, where $x$ is a fixed real number and $n$ ranges over the naturals, is all of $X$. No finite number of these opens covers $X$, hence $X$ is not compact.

(3) Assuming $x=y \vee \neg(x=y)$ for any pair of reals, there is, for any real $x$, a well-defined continuous function from $X$ to (the localic version of) the naturals which, on the level of points, would map a surjection $f: \mathbb{N} \rightarrow \mathbb{R}$ to the smallest number $n$ such that $f(n)=x$.

(4) There is a well-defined continuous function from $X$ to (the localic version of) the reals which, on the level of points, would map a surjection $f$ to the number $\sum_{n=0}^{\infty} 2^{-n} \arctan (f(n))$. Unlike the previous example, this function is not locally constant.

(5) The locale $X$ is a sublocale of the locale $Y$ of arbitrary functions $\mathbb{N} \rightarrow \mathbb{R}$ (which can also be realized as a topological space). It can be obtained as the intersection of the uncountably many sublocales $Y_{x}$, where $Y_{x}$ is the sublocale of $Y$ consisting of those functions which hit the real $x$.

(6) A certain sublocale of $X$, the locale $X^{\prime}$ of those functions $\mathbb{N} \rightarrow \mathbb{R}$ for which any real has infinitely many preimages, has a fractal nature: It is covered by the (intersections with $X^{\prime}$ of the) opens $U_{0 x}$, where $x$ ranges over the reals; the pairwise intersection of these opens is (truly) empty; and they are each isomorphic to $X^{\prime}$ - on the level of points, by mapping a surjection $f$ in $U_{0 x}$ to the surjection $(n \mapsto f(n+1))$.

There is nothing special about the real numbers in this example; in fact, the example works just as well with any set $M$ in place of the reals. For any set $M$, there is a locale of surjections $\mathbb{N} \rightarrow M$, and this locale is trivial (isomorphic to the empty locale) if and only if $M$ is empty. Locales of these kind are used as an important reduction step in the extension

be uncountable 36. 
of Grothendieck's Galois theory by André Joyal and Myles Tierney [37, Section V.3].

Intersection of dense sublocales. In ordinary topology, the intersection of dense subspaces need not be dense. A simple example is the intersection of $\mathbb{Q}$ with its complement in $\mathbb{R}$. In contrast, the intersection of (even an arbitrary set of) dense sublocales is always again dense - even if the intersection might well have no points.

For instance, the locale-theoretic intersection of (the localic version of) $\mathbb{Q}$ with its complement does not have any points and is dense in (the localic version of) the reals. Intuitively, while these two sublocales do not have any points in common, there still is nontrivial "localic glue".

Another example is given by item (5) above: Each of the sublocales $Y_{x}$ is dense in $Y$, hence their intersection $X$ is so as well.

The Banach-Tarski paradox. The Banach-Tarski paradox is the unintuitive statement that a three-dimensional solid ball in $\mathbb{R}^{3}$ of radius $r$ can be partitioned into five disjoint subsets in such a way that rearranging those subsets using only Euclidean motions yields two disjoint solid balls of radius $r$ each. The axiom of choice is required to procure these subsets, and the Banach-Tarski paradox is not in contradiction with the basic properties of the Lebesgue measure in $\mathbb{R}^{3}$ because these intermediate subsets are not measurable.

The traditional way to avoid the Banach-Tarski paradox is to adopt the axiom of determinacy instead of the axiom of choice. Just as the axiom of choice posits that a certain property of the finite domain also holds for the infinite, the axiom of determinacy is a certain statement whose finitary analogue is provable in unadorned Zermelo-Fraenkel set theory. It entails that all subsets of $\mathbb{R}^{n}$ are measurable.

The Banach-Tarski paradox can also be avoided by adopting a localic point of view: While the localic counterparts of the five pieces do not have any points in common, the locale-theoretic pairwise intersections are still nontrivial [38]. Hence there is no paradox, as one would not expect a rearrangement of overlapping sublocales to preserve volume.

Random sequences. Cantor space is the space $2^{\mathbb{N}}$ of infinite binary sequences, equipped with the product topology. A subset $U \subseteq 2^{\mathbb{N}}$ is open with respect to this topology if and only if for every $\alpha \in U$, there is a 
number $n \in \mathbb{N}$ such that

$$
\left\{\beta \in 2^{\mathbb{N}} \mid \forall i<n . \alpha(i)=\beta(i)\right\} \subseteq U .
$$

The open subsets of $2^{\mathbb{N}}$ are precisely those subsets which encode observable (or semidecidable) properties of binary sequences, those properties for which a finite record of digits suffices to verify that a sequence has it (but not necessarily to falsify it). For instance, the sets $V=\left\{\alpha \in 2^{\mathbb{N}} \mid \alpha(0)=\right.$ $\alpha(1)=1\}$ and $W=\left\{\alpha \in 2^{\mathbb{N}} \mid \exists n \in \mathbb{N} . \alpha(n)=1\right\}$ are open and the sets

$$
\begin{aligned}
& A:=\left\{\alpha \in 2^{\mathbb{N}} \mid \forall n \in \mathbb{N} . \alpha(n)=0\right\} \\
& B:=\left\{\alpha \in 2^{\mathbb{N}} \mid \lim _{n \rightarrow \infty} \frac{1}{n} \sum_{i=0}^{n-1} \alpha(i)=\frac{1}{2}\right\}
\end{aligned}
$$

are not. With respect to the standard Lebesgue measure on $2^{\mathbb{N}}$, the sets $V$ and $W$ have measure $\frac{1}{4}$ and 1 , respectively.

Unlike a regular sequence such as $(0,0,0, \ldots)$, we intuitively expect that, if the digits of a random sequence are revealed step by step, we will eventually verify that it is an element of $W$; and moreover, that it has any observable property $U$ of Lebesgue measure 1 .

Elevating this expectation to a definition, it seems prudent to declare that a sequence is random if and only if it is contained in the intersection of all open subsets $U \subseteq 2^{\mathbb{N}}$ with Lebesgue measure 1 . One could then endeavor to setup a develop a theory of probability on this notion.

However, the resulting theory will be trivial for the plain reason that according to this definition, there are no random sequences: For any sequence $\alpha$, the set $\left\{\beta \in 2^{\mathbb{N}} \mid \exists n \in \mathbb{N} . \alpha(n) \neq \beta(n)\right\}$ is open, has measure 1 and does not contain $\alpha$.

Despite this fundamental issue, one can still argue that this approach has merit [38, and hence it is worthwhile to find a mathematical foundation which is capable of formalizing it. Topological spaces are too restrictive, but Alex Simpson observed that locales are sufficiently flexible: There is a locale of random sequences, defined as the locale-theoretic intersection of all measure-1 opens of $2^{\mathbb{N}}$. This locale is nontrivial despite having no points.

\subsection{Constructive concerns}

By relinquishing points, locales provide a more flexible notion of space. Section 1.1] substantiates this observation with several examples of nontrivial locales without any points. Constructive mathematics gives a further, orthogonal motivation to study locales: There are situations in which the 
relevant spaces do have enough points, but only if one subscribes to the axiom of choice or similar non-constructive principles. In these situations, the pointfree approach facilitated by locales helps to give constructive versions of classical results. We will further discuss this theme in Section 2.2 Examples for this phenomenon include the following.

Compactness of the unit interval. The unit interval, when realized as a topological space, can fail to be compact in constructive mathematics. For instance, it fails in the Russian school because the Kleene tree provides a computable open covering of $[0,1]$ with no computable finite subcovering (a self-contained exposition of this phenomenon is contained in Ref. [39]).

In contrast, the localic version of the unit interval is always compact. The proof is by an explicit computation with its basic opens, exploiting the inductive nature of derivations [40].

Similarly, Tychonoff's theorem that the product of any number of compact topological spaces is again compact is equivalent to the axiom of choice. Its localic counterpart, the statement that the product of any number of compact locales is again compact, can be verified without any nonconstructive principles [41.

Galois theory. Let $L \mid k$ be a Galois extension. The fundamental theorem of (infinite) Galois theory states that there is a bijection between the intermediate extensions $L|E| k$ and the closed subgroups of the topological Galois group $\operatorname{Gal}(L \mid k)$. The bijection maps an intermediate extension $L|E| k$ to the subgroup $\operatorname{Gal}(L \mid E)$ and its inverse maps a closed subgroup $H \subseteq \operatorname{Gal}(L \mid k)$ to the fixed field $L^{H}$.

Much of the proof of the fundamental theorem of Galois theory is constructive, but some parts use the law of excluded middle and Zorn's lemma in order to construct certain extensions of given field homomorphisms $\mathrm{f}$ As a consequence, the fundamental theorem of Galois theory as stated is not provable in constructive mathematics.

However, this failure is not for fundamental Galois-theoretic reasons, but because of an unfortunate choice in the definitions. There is a notion of a localic group 42 (a locale $G$ equipped with continuous maps $G \times G \stackrel{\circ}{\rightarrow}$ $G, G \stackrel{(\cdot)^{-1}}{\longrightarrow} G, 1 \stackrel{e}{\rightarrow} G$ satisfying the group axioms), and the topological ${ }^{\mathrm{f}}$ For instance, the statement $E \subseteq L \mathrm{Gal}(L \mid E)$ is trivial. For the converse inclusion, let $x \in$ $L^{\mathrm{Gal}(L \mid E)}$. Assume for the sake of contradiction that $x \notin E$. Using Zorn's lemma and the law of excluded middle, we find a homomorphism $\sigma: L \rightarrow L$ with $\left.\sigma\right|_{E}=$ id and $\sigma(x) \neq x$. This is a contradiction to $x \in L^{\operatorname{Gal}(L \mid E)}$. 
Galois group has a localic counterpart. The fundamental theorem can be reformulated to refer to this localic Galois group, and the proof of this reformulation is entirely constructive 43 .

If one is so inclined, then one can obtain the topological version of the fundamental theorem as a corollary of the localic version; the required nonconstructive principles for this step are neatly packaged up in the study of the relation of the localic Galois group with the topological one.

Remark 1. Incidentally, the fundamental theorem of Galois theory also showcases a related general phenomenon, namely that classical mathematics allows to push back topological concerns for a bit longer, whereas in constructive mathematics we have to embrace topology (in a sufficiently pointfree form such as locales) from the beginning.

To be more specific, classically, the basic version of the fundamental theorem (intermediate extensions correspond to subgroups) only holds for finite Galois extensions. For infinite Galois extensions, we have to restrict to closed subgroups. Constructively, the basic version cannot even be shown for finite extensions; we have to employ spatial language even for those, or else settle for a discrete version of the fundamental theorem: For finite field extensions, finite intermediate extensions correspond to finite subgroups [26, Theorem 8.8]. Classically, intermediate extensions of finite extensions and subgroups of finite groups are automatically finite, but constructively this can fail $g$

Gelfand duality. The celebrated Gelfand correspondence states that the mapping

$$
X \longmapsto \operatorname{Hom}(X, \mathbb{C})=\{f: X \rightarrow \mathbb{C} \mid f \text { is continuous }\}
$$

defines a contravariant equivalence between compact Hausdorff spaces and commutative unital $C^{\star}$-algebras. In the other direction, a $C^{\star}$-algebra $A$ is mapped to its maximal spectrum $\operatorname{Max}(A)$, the topological space of multiplicative linear functionals $A \rightarrow \mathbb{C}$.

Some parts of the proof require the axiom of choice, (only) for fabricating appropriate elements of the maximal spectrum. But as with the fundamental theorem of infinite Galois theory, the Gelfand correspondence

g For instance, the field extension $\mathbb{Q}(\sqrt{2}) \mid \mathbb{Q}$ is finite. However, if the intermediate extension $E:=\{x \in \mathbb{Q}(\sqrt{2}) \mid x \in \mathbb{Q} \vee \varphi\}$ is finite, then $\varphi \vee \neg \varphi$. Using the sheaf semantics reviewed in Section 2.4 this intermediate extension can be turned into a topological counterexample [42, page 65]. 
has a constructive core, and a pointfree reformulation unearths this constructive content: There is a contravariant equivalence between compact completely regular locales and commutative unital $C^{\star}$-algebras [44 46].

The constructive Gelfand correspondence has been used to construct phase spaces for quantum-mechanical systems in the Bohr topos approach to quantum mechanics [47,49]. Briefly, a quantum mechanical system is described by a $C^{\star}$-algebra $A$ which is in all interesting cases noncommutative. As such the Gelfand correspondence cannot be applied to $A$. But there is a mirror image of $A$ as a sheaf model on a certain locale, the Bohr topos of $A$, which is commutative. Since the universe of sheaves supports intuitionistic reasoning, as we will review in Section 2.4, the constructive Gelfand correspondence can then be applied to this mirror image.

Remark 2. For a time, we only knew that ZFC proved that there is a constructive proof of the localic version of Gelfand duality without directly knowing such a constructive proof. This situation arose because the first proof of the localic duality 44 employed Barr's theorem, to be reviewed on page 25.

\subsection{The basics of the theory of locales}

The starting point of the theory of locales is the following observation. The set $\mathcal{O}(X)$ of open subsets of a topological space $X$ forms a partially ordered set which has

$$
\begin{gathered}
\text { arbitrary joins (suprema) and finite meets (infima), } \\
\bigvee
\end{gathered}
$$

and where finite meets distribute over arbitrary joins:

$$
U \wedge \bigvee_{i} V_{i}=\bigvee_{i}\left(U \wedge V_{i}\right)
$$

The key idea of locales is to elevate this observation to a definition, in the process dropping the requirement for the elements of the ordered set to be sets of points:

Definition 3. A frame is a partially ordered set with (arbitrary, setindexed) joins and finite meets such that the distributive law holds. A frame homomorphism $\alpha: A \rightarrow A^{\prime}$ is a monotone map $A \rightarrow A^{\prime}$ which preserves arbitrary joins and finite meets.

The least element of a frame (the empty join) is denoted " $\perp$ " and the largest element (the empty meet) "T". The notion of a frame is (infinitarily) 
algebraic. To obtain a geometric notion, we "reverse the direction of the arrows":

Definition 4. A locale $X$ is given by a frame $\mathcal{O}(X)$, the "frame of opens of $X$ ". A morphism $f: X \rightarrow X^{\prime}$ of locales (or "continuous map of locales") is a frame homomorphism $\mathcal{O}\left(X^{\prime}\right) \rightarrow \mathcal{O}(X)$.

In place of the open sets of points, locales have arbitrary opens, the elements of their underlying frame. The opens of locales behave similar to the open sets in topology in that arbitrary unions and finite intersections make sense; but unlike before, they need not be sets of points. Occasionally we abuse notation and denote the largest open of a locale $X$ by " $X$ ".

Examples for locales include the following.

(1) Any topological space $Y$ induces a locale $L(Y)$ by setting $\mathcal{O}(L(Y)):=$ $\mathcal{O}(Y)$. A continuous map $f: Y \rightarrow Y^{\prime}$ of topological spaces induces the frame homomorphism $\mathcal{O}\left(Y^{\prime}\right) \rightarrow \mathcal{O}(Y), U \mapsto f^{-1}[U]$ in the other direction and hence a morphism $L(Y) \rightarrow L\left(Y^{\prime}\right)$ of locales in the same direction.

(2) The one-point locale pt is the locale induced by the one-point topological space $\{\star\}$. Its frame of opens is the powerset of $\{\star\}$, also known as the set $\Omega$ of truth values. Its least element is $\perp=\emptyset$ and its largest element is $T=\{\star\}$, and potentially not all elements of $\Omega$ are equal to one of these two.

(3) The locale of surjections $\mathbb{N} \rightarrow \mathbb{R}$ and the localic version of the reals of Section 1.1 are best constructed as classifying locales, a notion to be introduced in Section 2.2

(4) The radical ideals of any ring $A$ form a frame and hence give rise to a locale. We will identify this locale in Theorem 40 to be the spectrum of $A$ as studied in algebraic geometry.

Several notions in topology only refer to open sets and not to points. Such notions have an immediate counterpart in locale theory. For instance, a locale $X$ is compact iff for any family $\left(U_{i}\right)_{i \in I}$ of opens of $X$ such that $T=\bigvee_{i \in I} U_{i}$, there is a (Kuratowski-)finite subset $I^{\prime} \subseteq I$ such that $\mathrm{T}=\bigvee_{i \in I^{\prime}} U_{i}$. With this definition, a topological space $Y$ is compact iff its induced locale $L(Y)$ is.

\footnotetext{
${ }^{\mathrm{h}} \mathrm{A}$ set $M$ is Kuratowski-finite iff there is a surjection $[n] \rightarrow M$, where $[n]=\{1,2, \ldots, n\}$. In constructive mathematics, we distinguish this notion from the stronger condition for a set $M$ to be Bishop-finite, which requires that there is a bijection $[n] \rightarrow M$.
} 
Points do not appear in the definition of a locale, but they can be defined as a derived concept:

Definition 5. A point of a locale $X$ is a locale morphism pt $\rightarrow X$.

This definition is inspired from the situation with topological spaces, where continuous maps from the one-point space to a topological space $Y$ are in canonical bijection with the points of $Y$.

The underlying frame homomorphism $\alpha: \mathcal{O}(X) \rightarrow \Omega$ of a point $x$ of $X$ can be pictured as mapping each open $U$ of $X$ to the truth value to which extent $x$ belongs to $U$. Mnemonically, we write $x \notin U$ iff $\alpha(U)=\top$.

The point $x$ is completely determined by the information to which opens it belongs, that is by the set $\{U \in \mathcal{O}(X) \mid \alpha(U)=\top\}$. This set is a completely prime filter, that is a subset $\mathfrak{f} \subseteq \mathcal{O}(X)$ which is upward-closed, closed under finite meets and for which $\bigvee_{i} U_{i} \in \mathfrak{f}$ implies $U_{i} \in \mathfrak{f}$ for some index $i$; and conversely, any such completely prime filter gives rise to a point of $X$ (Exercise [7).

Definition 6. A locale $X$ is spatial if and only if its points suffice to detect the inclusion relation on the opens of $X$, that is if for any opens $U, V \in$ $\mathcal{O}(X)$, if $x \in U \Rightarrow x \in V$ for all points $x$ of $X$, then $U \preceq V$.

An equivalent definition is: A locale $X$ is spatial if and only if its points suffice to distinguish its opens, that is if for any opens $U, V \in \mathcal{O}(X)$, if $x \notin$ $U \Leftrightarrow x \in V$ for all points $x$ of $X$, then $U=V$.

For instance, any locale induced by a topological space is spatial. The locale of surjections $\mathbb{N} \rightarrow \mathbb{R}$ is a striking example of a locale which fails to be spatial. The localic real line is spatial in classical mathematics and can fail to be spatial in constructive mathematics (Exercise 22). In this case, the locale induced by the topological space of reals should not be confused with the true localic real line. These locales have the same points, but the former might not be locally compact while the latter always is.

Remark 7. The set of points of a locale $X$ can be made into a topological space, giving rise to a functor $\mathrm{Pt}:$ Loc $\rightarrow$ Top. This functor is right adjoint to the functor $L:$ Top $\rightarrow$ Loc. A locale $X$ is spatial iff the canonical morphism $L(\operatorname{Pt}(X)) \rightarrow X$ is an isomorphism, and a topological space $Y$ is sober iff the canonical morphism $Y \rightarrow \operatorname{Pt}(L(Y))$ is a homeomorphism. The space $\operatorname{Pt}(L(Y))$ is the sobrification of $Y$; for instance, the sobrification of any inhabited indiscrete space is the one-point space. 
The adjunction $L \dashv \mathrm{Pt}$ restricts to an equivalence between sober topological spaces and spatial locales. Assuming the law of excluded middle, both Hausdorff spaces and the schemes from algebraic geometry are sober (Exercise 3), hence the spaces of a wide range of the mathematical landscape can be faithfully studied as locales.

Remark 8. Since continuous maps between spaces induce locale morphisms, we have a canonical map $\operatorname{Hom}_{\text {Top }}\left(Y, Y^{\prime}\right) \rightarrow \operatorname{Hom}_{\text {Loc }}\left(L(Y), L\left(Y^{\prime}\right)\right)$. If $Y^{\prime}$ is sober, this map is a bijection. In particular, in this case the settheoretic points of $Y^{\prime}$ and the locale-theoretic points of $L\left(Y^{\prime}\right)$ are in canonical one-to-one correspondence.

\section{Exercises}

Exercise 1 (The space of points of a locale). Devise a canonical topology on the set of points of a locale.

Exercise 2 (Sober topological spaces as locales). Verify the statements made in Remark 8

Exercise 3 (Hausdorff spaces are sober).

A meet-irreducible open subset of a topological space $Y$ is an open subset $W \subseteq Y$ such that $(U \cap V \subseteq W) \Rightarrow(U \subseteq W) \vee(V \subseteq W)$ for all open subsets $U, V \subseteq Y$ and such that $Y \nsubseteq W$.

(a) Assuming the law of excluded middle, show that the completely prime filters of a topological space are in canonical one-to-one correspondence with the meet-irreducible open subsets.

Hint. Given a completely prime filter $\mathfrak{f} \subseteq \mathcal{O}(Y)$, the set $\bigcup\{U \in \mathcal{O}(Y) \mid U \notin \mathfrak{f}\}$ is a meet-irreducible open subset.

(b) Assuming the law of excluded middle, show that a topological space is sober in the sense of Remark 7 if and only if for every meet-irreducible open subset $W$ there is a unique point $y \in Y$ such that $W=Y \backslash \overline{\{y\}}$. (This is a classical definition of sobriety.)

(c) Assuming the law of excluded middle, show that Hausdorff topological spaces validate the condition stated in part (b) and hence are sober.

Note. Without the law of excluded middle, Hausdorff spaces and even metric spaces may fail to be sober. In fact, already the topological space of rational numbers can fail to be sober [50 Example 8.14(iv)].

Exercise 4 (Complete metric spaces are sober). A metric space is a set $Y$ together with a relation $d \subseteq Y \times Y \times \mathbb{Q}_{\geq 0}$, written " $d(x, y) \leq q$ ", 
such that the following axioms are satisfied.

$$
\begin{aligned}
d(x, y) \leq q \wedge q \leq r \Longrightarrow & d(x, y) \leq r \\
\left(\forall r \in \mathbb{Q}_{>q} . d(x, y) \leq r\right) \Longrightarrow & d(x, y) \leq q \\
& \exists q \in \mathbb{Q}_{\geq 0} . d(x, y) \leq q \\
& d(x, x) \leq 0 \\
d(x, y) \leq 0 \Longrightarrow & x=y \\
d(x, y) \leq q \Longrightarrow & d(y, x) \leq q \\
d(x, y) \leq q \wedge d(y, z) \leq r \Longrightarrow & d(x, z) \leq q+r
\end{aligned}
$$

A Cauchy process (or multi-valued modulated Cauchy sequence) in a metric space $Y$ is a map $\alpha: \mathbb{Q}_{>0} \rightarrow P(Y)$ such that all the sets $\alpha(q)$ are inhabited and such that $d\left(x, x^{\prime}\right) \leq q+q^{\prime}$ for all $q, q^{\prime} \in \mathbb{Q}_{>0}$ and $x \in \alpha(q), x^{\prime} \in \alpha\left(q^{\prime}\right)$. Such a process converges to $x_{0} \in Y$ iff $d\left(x, x_{0}\right) \leq q$ for all $q \in \mathbb{Q}_{>0}$ and $x \in$ $\alpha(q)$. A metric space $Y$ is complete iff every Cauchy process in $Y$ converges.

Verify, without using the law of excluded middle, that any complete metric space is sober.

Note. In the presence of the countable axiom of choice, any Cauchy process can be refined to a Cauchy sequence. In its absence, however, the familiar completion construction using equivalence classes of Cauchy sequences fails to yield a Cauchy-complete space. In contrast, any Cauchy sequence and more generally any Cauchy process converges in the space of equivalence classes of Cauchy processes.

Exercise 5 (Isomorphisms of frames). Show that a frame homomorphism $\alpha: A \rightarrow A^{\prime}$ is an isomorphism (that is, admits an inverse frame homomorphism) if and only if $\alpha$ is surjective and reflects the ordering (that is, $\alpha(U) \preceq \alpha(V) \Rightarrow U \preceq V)$.

Exercise 6 (The covariant approach to locales). Let $A$ be a frame.

(a) Show that $A$ contains arbitrary (set-indexed) meets (though they are not required to be preserved by frame homomorphisms), by the impredicative construction

$$
\bigwedge_{i \in I} U_{i}=\bigvee\left\{V \in A \mid V \preceq U_{i} \text { for all } i \in I\right\} .
$$

(b) What are the arbitrary meets in the frame of open subsets of a topological space?

(c) Show that for any frame homomorphism $\alpha: A \rightarrow A^{\prime}$ there exists a monotone map $\beta: A^{\prime} \rightarrow A$ such that $\beta$ is right adjoint to $\alpha$, that is such that

$$
\alpha(U) \preceq V \quad \text { iff } \quad U \preceq \beta(V)
$$

for all $U \in A$ and $V \in A^{\prime}$. 
Note. Based on the observation in part (c), a covariant approach to locales can be developed, in which locale maps $X \rightarrow X^{\prime}$ are defined to be certain kinds of maps $\mathcal{O}(X) \rightarrow \mathcal{O}\left(X^{\prime}\right)$ 51.

Exercise 7 (Points as complete prime filters). Verify that the points of a locale $X$ are in canonical one-to-one correspondence with the completely prime filters of $\mathcal{O}(X)$.

Exercise 8 (Dense opens). An open $U$ of a locale is dense iff for any open $V, U \wedge V=\perp$ implies $V=\perp$.

(a) Let $M$ be a set and endow it with the discrete topology. Show that an open $U \in \mathcal{O}(L(M))$ is dense iff for any element $x \in M, \neg \neg(x \in U)$.

(b) Let $\varphi$ be a truth value, hence an open of the one-point locale pt. Show that $\varphi$ is dense in pt iff $\neg \neg \varphi$.

Exercise 9 (Characterizing spatial locales). Let $X$ be a locale. Show that the following conditions are equivalent.

(a) For any opens $U, V \in \mathcal{O}(X)$ : If $x \notin U \Rightarrow y \in V$ for all points $x$ of $X$, then $U \preceq V$.

(b) For any opens $U, V \in \mathcal{O}(X)$ : If $x \notin U \Leftrightarrow y \notin V$ for all points $x$ of $X$, then $U=V$.

(c) There is a topological space $Y$ and a surjective morphism $L(Y) \rightarrow X$ of locales. (A morphism of locales is surjective iff its underlying frame homomorphism is injective.)

(d) The canonical morphism $L(\operatorname{Pt}(X)) \rightarrow X$ is an isomorphism.

Exercise 10 (The frame of radical ideals). Let $A$ be a ring. We order the set $\operatorname{Idl}(A)$ of ideals of $A$ by inclusion. (See Section 3.1 for some algebraic preliminaries.)

(a) Verify that $\operatorname{Idl}(A)$ has arbitrary meets and joins, given by intersection and sum of ideals, but that it is in general not a frame. Hint. The distributive law fails in the polynomial ring $\mathbb{Q}[X, Y]$.

(b) Show that the sub-poset $\operatorname{Rad}(A)$ of radical ideals is a frame, with the same finite meets but with joins given by the radical of the join computed in $\operatorname{Idl}(A)$.

(c) Let $\varphi$ be a truth value. Let $\{\top \mid \varphi\}$ be the subsingleton subset of $\operatorname{Rad}(A)$ which contains the top element $\sqrt{(1)}$ iff $\varphi$. Verify that

$$
\bigvee\{\top \mid \varphi\}=\{f \in A \mid f \text { is nilpotent or } \varphi\}
$$

in $\operatorname{Rad}(A)$. 
Exercise 11 (Coproduct of locales). (a) Show that the category of frames has small products.

(b) Deduce that the category of locales has small coproducts.

Note. The category of locales also has small products; Exercise20 is devoted to this fact.

(c) Let $M$ be a set and endow it with the discrete topology. Show that $L(M)$ is the coproduct $\amalg_{x \in M}$ pt.

Exercise 12 (Local locales). A locale $X$ is local iff for any open covering $T=\bigvee_{i} U_{i}$, there is an index $i$ such that $T=U_{i}$. (For instance, the spectrum of a ring $A$ is local iff $A$ is local.) Show that any local locale has a canonical point, its focal point.

Exercise 13 (Maps into discrete spaces). Let $X$ be a locale. Let $A$ be a set and endow it with the discrete topology. Show that morphisms $X \rightarrow$ $L(A)$ are in canonical correspondence with families $\left(U_{a}\right)_{a \in A}$ of opens of $X$ such that $\top=\bigvee_{a \in A} U_{a}$ and $U_{a} \wedge U_{b} \preceq \bigvee\{\top \mid a=b\}$

\section{Geometric theories and sheaf models}

\subsection{Geometric theories}

Definition 9. A geometric theory consists of

(1) a set of sorts: $X, Y, Z, \ldots$

(2) a set of function symbols: $f: X \times Y \rightarrow Z, \ldots$

(3) a set of relation symbols: $R \hookrightarrow X \times Y \times Z, \ldots$

(4) a set of geometric sequents as axioms: $\varphi \vdash_{x: X, y: Y} \psi, \ldots$

A geometric sequent (in some context $x_{1}: X_{1}, \ldots, x_{n}: X_{n}$ ) is a formula built using only the ingredients $=\top \wedge \perp \vee \vee \exists$ and the relation symbols (but no $\Rightarrow \forall)$. We follow the usual convention that negation is understood as syntactic sugar for implication to $\perp$. The symbol " $\bigvee$ " refers to disjunction of arbitrary set-indexed families of formulas. The theory proves a sequent $\sigma$ if and only if there exists a derivation of $\sigma$, where the class of derivations is inductively generated by the axioms and the rules displayed in Table 1 il

We refer to Ref. [12, Section D1.1] for a more detailed version of Definition 9

iBecause of the infinitary disjunctions, some care is needed to not "build in" the axiom of choice in the definition of derivations. More specifically, we allow inhabited sets of subdeductions instead of requiring that subdeductions are given by a function. Details on the implementation in CZF can be found in Ref. [52, Definition 5.2]. 
Table 1. The rules of geometric logic

$$
\begin{aligned}
& \frac{\varphi \vdash_{\vec{x}} \psi{ }^{\text {structural rules }}}{\varphi \vdash_{\vec{x}} \varphi} \quad \frac{\varphi \vdash_{\vec{x}} \chi}{\varphi[\vec{s} / \vec{x}] \vdash_{\vec{y}} \psi[\vec{s} / \vec{x}]}{ }^{(\vec{y} \text { not bound })} \\
& \text { rules for conjunction } \\
& \overline{\varphi \vdash_{\vec{x}} \top} \frac{}{\varphi \wedge \psi \vdash_{\vec{x}} \varphi} \frac{}{\varphi \wedge \psi \vdash_{\vec{x}} \psi} \quad \frac{\varphi \vdash_{\vec{x}} \psi}{\varphi \vdash_{\vec{x}} \psi \wedge \chi} \\
& \text { rules for finitary disjunction } \\
& \frac{}{\perp \vdash_{\vec{x}} \varphi} \frac{}{\varphi \vdash_{\vec{x}} \varphi \vee \psi} \frac{\varphi \vdash_{\vec{x}} \chi \quad \psi \vdash_{\vec{x}} \chi}{\varphi \vdash_{\vec{x}} \varphi \vee \psi} \frac{\vdash_{\vec{x}} \chi}{\varphi} \\
& \text { rules for infinitary disjunction } \\
& \begin{array}{c}
\frac{\varphi_{i} \vdash_{\vec{x}} \chi \text { for each } i \in I}{\bigvee_{i_{0}} \vdash_{\vec{x}} \bigvee_{i \in I} \varphi_{i} \vdash_{\vec{x}} \chi} \\
\text { double rule for existential quantification }
\end{array} \\
& \frac{\varphi \vdash_{\vec{x}, y} \psi}{\exists y: Y \cdot \varphi \vdash_{\vec{x}} \psi}(y \text { not free in } \psi)
\end{aligned}
$$

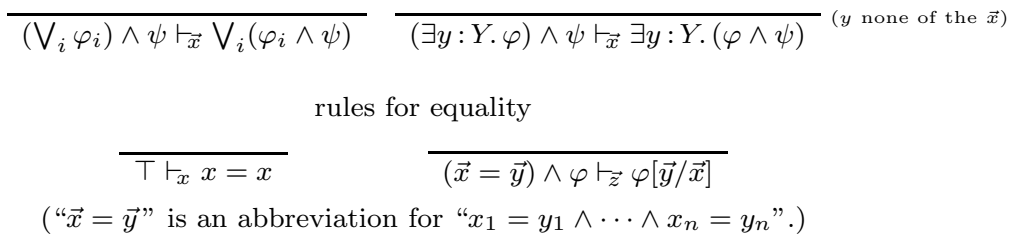

Even though superficially similar, geometric theories play a substantially different role than formal systems such as Peano arithmetic or ZermeloFraenkel set theory. There are a number of notable differences:

(1) Formal systems are typically of foundational interest and can be fruitfully employed as metatheories. Geometric theories are more interesting for specific applications, for instance for presenting spaces. This aspect is discussed in Section 2.2 ,

(2) Formal systems typically come with an intended model; geometric theories do not. For instance, the geometric theory of groups has all groups as models; when we are writing down the axioms of a group, we are not setting out to capture the properties of any one specific group.

(3) Formal systems are typically required to be recursively axiomatizable. 
Table 2. The rules of infinitary intuitionistic first-order logic (structural rules and rules for $=\top \wedge \perp \vee \vee \exists$ as in Table 1)

rules for infinitary conjunction

$$
\begin{gathered}
\frac{\frac{\psi \vdash_{\vec{x}} \varphi_{i} \text { for each } i \in I}{\psi \vdash_{\vec{x}} \bigwedge_{i \in I} \varphi_{i}}}{\bigwedge_{i \in I} \varphi_{i} \vdash_{\vec{x}} \varphi_{i_{0}}} \quad \frac{}{\text { double rule for implication }} \\
\frac{\varphi \wedge \psi \vdash_{\vec{x}} \chi}{\varphi \vdash_{\vec{x}} \psi \Rightarrow \chi}
\end{gathered}
$$$$
\text { double rule for universal quantification }
$$$$
\frac{\varphi \vdash_{\vec{x}, y} \psi}{\varphi \vdash_{\vec{x}} \forall y: Y \cdot \psi}(y \text { not free in } \varphi)
$$

Many interesting geometric theories are not, and indeed contain an uncountable number of sorts, function symbols, relation symbols or axioms. Geometric theories also allow for arbitrary set-indexed disjunctions; for this to make sense, the study of geometric theories can only be carried out in a sufficiently rich background theory.

(4) For formal systems, typically a version of Gödel's completeness theorem holds: A formula is derivable iff it holds in all models. In contrast, geometric theories can be consistent yet do not admit any set-based models. An example is the geometric theory of surjections $\mathbb{N} \rightarrow \mathbb{R}$ reviewed below.

(5) Perhaps the most important difference to formal systems is that geometric theories, unlike formal systems, often depend on given mathematical objects. For instance, for each ring $A$, there is the geometric theory of prime filters of $A$. This theory will play a crucial role in Section 3 .

Definition 10. A set-based model $M$ (or "Tarski model") of a geometric theory $\mathbb{T}$ consists of

(1) a set $\llbracket X \rrbracket$ for each sort $X$,

(2) a function $\llbracket f \rrbracket: \llbracket X_{1} \rrbracket \times \cdots \times \llbracket X_{n} \rrbracket \rightarrow \llbracket Y \rrbracket$ for each function symbol $f:$ $X_{1} \times \cdots \times X_{n} \rightarrow Y$ and

(3) a relation $\llbracket R \rrbracket \subseteq \llbracket X_{1} \rrbracket \times \cdots \times \llbracket X_{n} \rrbracket$ for each relation symbol $R \hookrightarrow$ $X_{1} \times \cdots \times X_{n}$

such that $M$ validates the axioms of $\mathbb{T}$. 
We explicitly allow empty carriers, that is we do not demand that the sets $\llbracket X \rrbracket$ are inhabited. Examples for geometric theories include the following.

(1) The geometric theory of rings. This theory has one sort, $R$; five function symbols: 0 and 1 (nullary), - (unary), + and - (binary); no relation symbols; and the usual axioms, such as $\top \vdash_{x: R, y: R} x+y=y+x$.

A set-based model of the theory of rings is an ordinary ring.

By adding the axiom

$$
\top \vdash_{x: R}(x=0) \vee(\exists y: R \cdot x y=1),
$$

we describe geometric fields, and by additionally adding for each natural number $n \geq 1$ the axiom

$$
\top \vdash_{a_{0}: R, \ldots, a_{n-1}: R} \exists x: R . x^{n}+a_{n-1} x^{n-1}+\cdots+a_{1} x+a_{0}=0,
$$

we describe algebraically closed geometric fields. (This field condition is called "geometric" because it can be put as a geometric sequent. Other, constructively not equivalent conditions such as "any nonzero element is invertible" or "any nonunit is zero" are not geometric sequents.)

In a similar vein, there is the geometric theory of monoids, of groups, and so on. Some of these theories use the infinite disjunctions supported by geometric logic: For instance, the theory of fields of positive characteristic or the theory of torsion groups have among their axioms

$$
\top \vdash \bigvee_{n \in \mathbb{N}_{\geq 1}}(\underbrace{1+\cdots+1}_{n \text { summands }}=0) \quad \text { or } \quad \top \vdash_{g: G} \bigvee_{n \in \mathbb{N}}\left(g^{n}=e\right)
$$

(2) The geometric theory of objects. This theory has one sort, $X$, and no function symbols, relation symbols or axioms. A set-based model of this theory is just a set.

A variant of this theory is the theory of inhabited objects, which has the axiom $\top \vdash \exists x: X$. $\top$. Its set-based models are precisely the inhabited sets.

(3) The geometric theory of surjections $f: \mathbb{N} \rightarrow \mathbb{R}$. This theory has no sorts; no function symbols; a nullary relation symbol $\varphi_{n x}$ for each pair $\langle n, x\rangle \in \mathbb{N} \times \mathbb{R}$ (to be read as " $f$ maps $n$ to $x$ "); and the following axioms:

(a) For each $n \in \mathbb{N}$, the axiom $\top \vdash \bigvee_{x \in \mathbb{R}} \varphi_{n x}$, expressing that $f$ is total. 
(b) For each $n \in \mathbb{N}$ and each $x, y \in \mathbb{R}$, the axiom $\varphi_{n x} \wedge \varphi_{n y} \vdash \bigvee\{\top \mid x=$ $y$, expressing that $f$ is single-valued $[\mathrm{j}$

(c) For each $x \in \mathbb{R}$, the axiom $\top \vdash \bigvee_{n \in \mathbb{N}} \varphi_{n x}$, expressing that $f$ is surjective.

A model of this geometric theory consists of an $(\mathbb{N} \times \mathbb{R})$-indexed family of truth values, that is a subset $G$ of $\mathbb{N} \times \mathbb{R}$, satisfying precisely those axioms which render $G$ the graph of a surjection $\mathbb{N} \rightarrow \mathbb{R}$.

(4) The geometric theory of Dedekind cuts. This theory has no sorts; no function symbols; nullary relation symbols $\alpha_{x}$ and $\beta_{x}$ for each rational number $x$ (to be read as " $x$ is contained in the lower (upper) cut"); and the following axioms, spelling out that the cut is bounded, rounded, open and located:

$$
\begin{aligned}
& \top \vdash \bigvee_{x \in \mathbb{Q}} \alpha_{x} \\
& \top \vdash \bigvee_{x \in \mathbb{Q}} \beta_{x} \\
& \alpha_{y} \vdash \alpha_{x} \quad \text { (for each } x, y \in \mathbb{Q} \text { such that } x<y \text { ) } \\
& \beta_{x} \vdash \beta_{y} \quad \text { (for each } x, y \in \mathbb{Q} \text { such that } x<y \text { ) } \\
& \alpha_{x} \vdash \bigvee_{y>x} \alpha_{y} \quad \text { (for each } x \in \mathbb{Q} \text { ) } \\
& \beta_{y} \vdash \bigvee_{x<y} \beta_{x} \quad \text { (for each } y \in \mathbb{Q} \text { ) } \\
& \alpha_{x} \wedge \beta_{y} \vdash \perp \quad \text { (for each } x, y \in \mathbb{Q} \text { such that } x \geq y \text { ) } \\
& \top \vdash \alpha_{x} \vee \beta_{y} \quad \text { (for each } x, y \in \mathbb{Q} \text { such that } x<y \text { ) }
\end{aligned}
$$

A model of this geometric theory consists of two families of truth values, both indexed by the rational numbers, hence two subsets $L, U \subseteq \mathbb{Q}$, in such a way that $\langle L, U\rangle$ is a Dedekind cut.

(5) The geometric theory of prime ideals of a given ring $A$. In classical commutative algebra, the notion of a prime ideal of a ring $A$ is fundamental. Corresponding to the definition (recalled in Section 3.1), the geometric theory of prime ideals has no sorts; no function symbols; one relation symbol " $V(x)$ " for each element $x \in A$; and the following

\footnotetext{
${ }^{\mathrm{j}}$ The disjunction is taken over the set $\{\top \mid x=y\}$. This is a certain subsingleton set of formulas; it is inhabited (by the formula $T$ ) iff $x=y$. Hence, if $x=y$, this axiom reads $\varphi_{n x} \wedge \varphi_{n y} \vdash \top$ and could also be omitted; if $x \neq y$, this axiom reads $\varphi_{n x} \wedge \varphi_{n y} \vdash \perp$. If we work in a constructive metatheory, we cannot perform this case distinction.
} 
axioms:

$$
\begin{array}{rlrl}
\top & \vdash V(0) & & \\
V(x) \wedge V(y) & \vdash V(x+y) & & \text { (for each } x, y \in A) \\
V(x) & \vdash V(x y) & & \text { (for each } x, y \in A) \\
V(1) & \vdash \perp & & \text { (for each } x, y \in A) \\
V(x y) & \vdash V(x) \vee V(y) &
\end{array}
$$

For many purposes, the theory of prime filters is actually more relevant $\mathrm{k}$ Classically, a prime filter of a ring is simply the complement of a prime ideal; constructively, it is prudent to turn prime ideals upside down and axiomatize this notion directly. Hence the theory of prime filters of $A$ has no sorts, no function symbols, one relation symbol " $D(x)$ " for each element $x \in A$ and the following axioms:

$$
\begin{aligned}
D(0) & \vdash & & \\
D(x+y) & \vdash D(x) \vee D(y) & & \text { (for each } x, y \in A) \\
D(x y) & \vdash D(x) & & \text { (for each } x, y \in A) \\
\top & \vdash D(1) & & \\
D(x) \wedge D(y) & \vdash D(x y) & & \text { (for each } x, y \in A)
\end{aligned}
$$

(6) The geometric theory of automorphisms of a field. Let $L \mid k$ be a field extension. The theory of automorphisms $L \rightarrow L$ which fix $k$ has one nullary relation symbol " $\sigma_{x y}$ " for each pair $\langle x, y\rangle \in L$ and the following

\footnotetext{
${ }^{\mathrm{k}}$ This is not to say that all the issues with prime ideals in constructive mathematics evaporate when turning to prime filters. For a time it was believed that algebraic geometry could be developed constructively if only the spectrum of a ring was defined as the topological space of its prime filters [53 Section 3], but this hope turned out to be too naive. André Joyal gave an explicit example of a nontrivial sheaf model of the theory of rings without any prime filters [54 pp. 200f.].
} 
axioms:

$$
\begin{array}{rlr}
\top & \vdash \bigvee_{y} \sigma_{x y} & \text { (for each } x \in L) \\
\top & \vdash \bigvee_{x} \sigma_{x y} & \text { (for each } y \in L) \\
\sigma_{x y} \wedge \sigma_{x y^{\prime}} & \vdash \bigvee\left\{\top \mid y=y^{\prime}\right\} & \text { (for each } \left.x, y, y^{\prime} \in L\right) \\
\sigma_{x y} \wedge \sigma_{x^{\prime} y} & \vdash \bigvee\left\{\top \mid x=x^{\prime}\right\} & \text { (for each } \left.x, x^{\prime}, y \in L\right) \\
\top & & \\
\top & \vdash \sigma_{00} & \\
\sigma_{x y} \wedge \sigma_{x^{\prime} y^{\prime}} & \vdash \sigma_{x+x^{\prime}, y+y^{\prime}} & \text { (for each } \left.x, x^{\prime}, y, y^{\prime} \in L\right) \\
\sigma_{x y} \wedge \sigma_{x^{\prime} y^{\prime}} & \vdash \sigma_{x x^{\prime}, y y^{\prime}} & \text { (for each } \left.x, x^{\prime}, y, y^{\prime} \in L\right) \\
\top & \vdash \sigma_{x x} & \text { (for each } x \in k)
\end{array}
$$

A model of this theory (can be reorganized to be) an element of the classical Galois group of $L \mid k$.

(7) The inconsistent geometric theory. This theory has no sorts, function symbols or relations, but the single axiom $T \vdash \perp$. This theory does not admit any set-based models.

(8) The empty geometric theory. This theory does not have any sorts, function symbols, relations or axioms. There is exactly one set-based model of this theory, the empty structure.

Extracting constructive content from classical proofs. Geometric logic strikes a fine balance between being sufficiently expressive in order to have rich applications and also being sufficiently restrictive so that Barr's theorem holds. This theorem is concerned with eliminating classical reasoning from proofs of geometric sequents, and it comes in a simple yet still useful and a more sophisticated version:

(1) If infinitary classical logic (classical logic extended by set-indexed conjunctions and disjunctions) proves a geometric sequent, then so does geometric logic.

(2) If a specific version of extensional type theory with the law of excluded middle and the axiom of choice proves a geometric sequent, then so does geometric logic.

Barr's theorem has ramifications for constructive algebra, as pointed out by Gavin Wraith [55. For instance, the Nullstellensatz is the statement 
that for any system $f_{1}, \ldots, f_{n} \in K\left[X_{1}, \ldots, X_{m}\right]$ of polynomials over an algebraically closed field $K$, either the polynomials $f_{i}$ have a common zero or there are polynomials $g_{i}$ such that $1=\sum_{i} f_{i} g_{i}$ (such an expression is an algebraic witness of the nonexistence of a common zero).

The Nullstellensatz is a theorem of classical mathematics whose usual proof employs the axiom of choice. Since it can be formulated as a geometric sequent over the geometric theory of algebraically closed geometric fields, Barr's theorem implies that there is also a constructive proof, in fact even a purely geometric proof.

The simpler version of Barr's theorem is constructive, and although we will not use it in the remainder of this text, we use this opportunity to present a self-contained proof. The proof describes an explicit method for eliminating the law of excluded middle from proofs of geometric sequents.

In contrast, the full version is not constructive This puts us in the curious situation that we are promised, unconstructively, that there exist certain constructive proofs, without being given any indication how these proofs could be found or what an upper bound on their length is.

However, knowing that these proofs exist platonically is still worthwhile, as we can be assured that looking for these proofs is not bound to fail. Indeed, for the example of the Nullstellensatz, a constructive proof is given in Ref. [14, Theorem 9.7].

Also, if one is interested in constructive proofs not because of their algorithmic content or of the mathematical insights they communicate but more pragmatically for their applicability in, for instance, sheaf models, not having them explicitly available is entirely bearable.

For the precise statement of the simple version of Barr's theorem, we recall that the rules of geometric logic have been defined in Table 1 that the rules of infinitary intuitionistic first-order logic have been defined in Table 2, and that infinitary classical logic is infinitary intuitionistic logic extended by the law of excluded middle.

Theorem 11 (Barr's theorem, simple version). Let $\mathbb{T}$ be a geometric

\footnotetext{
1The textbook argument [56, Theorem 7.57] establishing the full version of Barr's theorem requires the axiom of choice. But recently Michael Rathjen was able to lower the required metatheory to ZF 52 Remark 4.2], by working internally to a relative version of Gödel's constructible universe $L$ over a forcing extension, where the Barwise completeness theorem applies. To our knowledge, the precise strength of Barr's theorem relative to CZF or IZF has not yet been precisely calibrated. In particular, it is an open question [57] whether Barr's theorem implies the law of excluded middle, though it would be a major surprise if it did not.
} 
theory. Let $\sigma$ be a geometric sequent over the signature of $\mathbb{T}$. Then the following statements are equivalent.

(1) There is a $\mathbb{T}$-derivation of $\sigma$ in geometric logic.

(2) There is a $\mathbb{T}$-derivation of $\sigma$ in infinitary intuitionistic logic.

(3) There is a $\mathbb{T}$-derivation of $\sigma$ in infinitary classical logic.

Proof. It is immediate that (1) implies (3).

To show that (3) implies (2), we combine the double negation translation with Friedman's "nontrivial exit continuation" trick. We give a sketch of the main thrust of the argument, omitting lengthy verifications. Let $\sigma \equiv$ $\left(\alpha \vdash_{\vec{a}} \beta\right)$. For notational simplicity, we assume that the context $\vec{a}$ is empty, though all of the following can be adapted to the nonempty case if care is taken to avoid variable capture.

We introduce the operator $\nabla$ with $\nabla \varphi: \equiv((\varphi \Rightarrow \beta) \Rightarrow \beta)$. This operator is a local operator [58, Section 14.5] in the sense that for any formula $\varphi$ in some context $\vec{x}$, infinitary intuitionistic logic proves the sequents

$$
\top \vdash_{\vec{x}}(\varphi \Rightarrow \nabla \varphi), \top \vdash_{\vec{x}}(\nabla \nabla \varphi \Rightarrow \nabla \varphi), \top \vdash_{\vec{x}}((\nabla \varphi \wedge \nabla \psi) \Leftrightarrow \nabla(\varphi \wedge \psi)) .
$$

We then set up the $\nabla$-translation $\varphi \mapsto \varphi^{\nabla}$ for infinitary first-order formulas over the signature of $\mathbb{T}$ as described in Table 3 .

Table 3. The definition of the $\nabla$-translation

$$
\begin{array}{rlrl}
(x=y)^{\nabla} & : \equiv \nabla(x=y) & & \\
R\left(x_{1}, \ldots, x_{n}\right)^{\nabla} & : \equiv \nabla\left(R\left(x_{1}, \ldots, x_{n}\right)\right) & & \\
\top^{\nabla} & : \equiv \top & & \\
\perp^{\nabla} & : \equiv \nabla \perp & & \left(\bigwedge_{i} \varphi_{i}\right)^{\nabla}: \equiv \bigwedge_{i} \varphi_{i}^{\nabla} \\
(\varphi \wedge \psi)^{\nabla} & : \equiv \varphi^{\nabla} \wedge \psi^{\nabla} & & \left(\bigvee_{i} \varphi_{i}\right)^{\nabla}: \equiv \nabla\left(\bigvee_{i} \varphi_{i}^{\nabla}\right) \\
(\varphi \vee \psi)^{\nabla} & : \equiv \nabla\left(\varphi^{\nabla} \vee \psi^{\nabla}\right) & \\
(\varphi \Rightarrow \psi)^{\nabla} & : \equiv\left(\varphi^{\nabla} \Rightarrow \psi^{\nabla}\right) & (\exists x: X . \varphi)^{\nabla}: \equiv \nabla\left(\exists x: X . \varphi^{\nabla}\right) \\
(\forall x: X . \varphi)^{\nabla} & : \equiv\left(\forall x: X . \varphi^{\nabla}\right) &
\end{array}
$$

Lengthy but straightforward computations by induction on the structure of formulas or derivations then establish the following properties.

(a) For any infinitary first-order formula $\varphi$ in some context $\vec{y}$, infinitary intuitionistic logic proves $\nabla\left(\varphi^{\nabla}\right) \vdash_{\vec{x}} \varphi^{\nabla}$.

(b) For any geometric formula $\varphi$ in some context $\vec{x}$, infinitary intuitionistic $\operatorname{logic}$ proves $\varphi^{\nabla} \dashv_{\vec{x}} \nabla \varphi$. 
(c) For any derivation of a sequent $\varphi \vdash_{\vec{x}} \psi$ in infinitary classical logic, there is a derivation of $\varphi^{\nabla} \vdash_{\vec{x}} \psi^{\nabla}$ in infinitary intuitionistic logic. This property rests on the fact that the $\nabla$-translation of the law of excluded middle,

$$
\top \vdash_{\vec{x}} \nabla\left(\varphi^{\nabla} \vee\left(\varphi^{\nabla} \Rightarrow \nabla \perp\right)\right),
$$

is intuitionistically provable.

Combining these ingredients, a given derivation of $\sigma \equiv(\alpha \vdash \beta)$ in infinitary classical logic can be transformed to give a derivation of

$$
\alpha \vdash \nabla \alpha \vdash \alpha^{\nabla} \vdash \beta^{\nabla} \vdash \nabla \beta \equiv((\beta \Rightarrow \beta) \Rightarrow \beta) \vdash \beta
$$

in infinitary intuitionistic logic.

To verify that (2) implies (1), we define a semantics " $\alpha \models \varphi$ " for geometric formulas $\alpha$ and infinitary first-order formulas $\varphi$ by the clauses in Table 4. For notational simplicity, we restrict to the propositional case, that is assume that the set of sorts of $\mathbb{T}$ is empty. The following properties of the semantics can then be established by induction:

(a) If $\mathbb{T}$ proves $\beta \vdash \alpha$ (in geometric logic), then $\alpha \models \varphi$ implies $\beta \models \varphi$.

(b) If $\mathbb{T}$ proves $\alpha \dashv \bigvee_{i} \beta_{i}$ (geometrically) and if $\beta_{i} \models \varphi$ for all indices $i$, then $\alpha=\varphi$.

(c) If $\varphi$ is a geometric formula, then $\alpha \models \varphi$ iff $\mathbb{T}$ proves $\alpha \vdash \varphi$ (geometrically).

(d) If a first-order sequent $(\varphi \vdash \psi)$ is derivable from the axioms of $\mathbb{T}$ in infinitary intuitionistic logic, then $\top \models(\varphi \Rightarrow \psi)$.

We can then conclude as follows. Assume that $(\varphi \vdash \psi)$ is derivable in infinitary intuitionistic logic. By (d) , we have $\top \models(\varphi \Rightarrow \psi)$. By the clause for implication in Table 4, we have $\varphi \models \psi$. By (드), there is a $\mathbb{T}$-derivation of $\varphi \vdash \psi$ in geometric logic.

Remark 12. The semantics set up in the proof of Theorem 11 coincides with the sheaf semantics of the classifying locale of $\mathbb{T}$ reviewed in Section 2.2 and Section 2.4. In the nonpropositional case, the semantics coincides with the slightly more general topos semantics of the classifying topos of $\mathbb{T}$ [59, Section 2]. The local operator defined in the proof cuts out a certain Boolean subtopos of the classifying topos. An alternative proof proceeds by cut elimination [52, Section 6]; see also Ref. [60, and Ref. 61] for background on proof-theoretical reduction, of which Barr's theorem is an example. 
Table 4. The semantics of the classifying topos of $\mathbb{T}$ (propositional case)

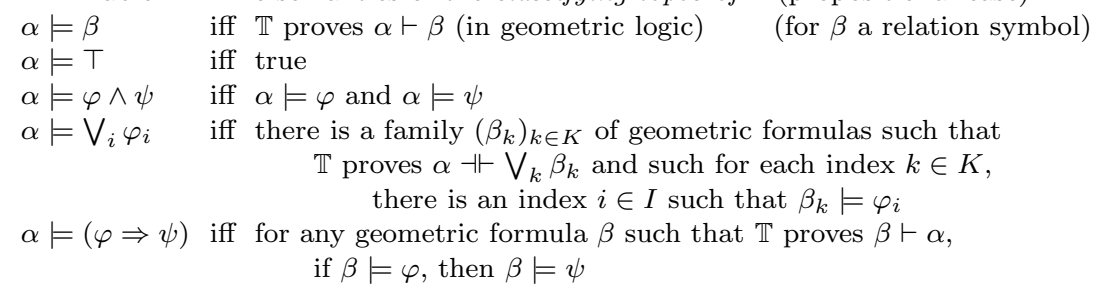

\subsection{Presenting frames by theories}

Definition 13. A geometric theory $\mathbb{T}$ is propositional if and only if its set of sorts is empty.

As a consequence, a propositional geometric theory consists just of a set of nullary relation symbols and a set of axioms. Associated to any such theory $\mathbb{T}$ is its Lindenbaum algebra: This is the partially ordered set of the geometric formulas over the signature of $\mathbb{T}$ modulo $\mathbb{T}$-provable equivalence, equipped with the ordering given by $[\varphi] \preceq[\psi]$ iff $\mathbb{T}$ proves $\varphi \vdash \psi$.

The Lindenbaum algebra is a frame, with finite meets given by $[\varphi] \wedge[\psi]=$ $[\varphi \wedge \psi]$ and set-indexed joins given by $\bigvee_{i}\left[\varphi_{i}\right]=\left[\bigvee_{i} \varphi_{i}\right] \mathrm{m}$ we can regard the Lindenbaum algebra as the free frame generated by the nullary relation symbols of $\mathbb{T}$ modulo the axioms of $\mathbb{T}$. Our interest in the Lindenbaum algebra is because it gives rise to a locale:

Definition 14. The classifying locale $L(\mathbb{T})$ of a propositional geometric theory is the locale which has the Lindenbaum algebra of $\mathbb{T}$ as its underlying frame.

It is an instructive exercise to verify that the points of $L(\mathbb{T})$ are in canonical one-to-one correspondence with the set-based models of $\mathbb{T}$. This observation also explains why we cannot hope to construct classifying locales of arbitrary, nonpropositional, geometric theories: Any locale only has a set of points, but arbitrary geometric theories can have a proper class of set-based models. (There is, however, a classifying arithmetic space 35] of any coherent theory and a classifying topos [59, Section 2] of any geometric theory.)

\footnotetext{
${ }^{m}$ This short description of the set-indexed joins picks representatives from each equivalence class and is hence only sensible in the presence of the axiom of choice. In a constructive metatheory, we should rather write $\bigvee_{i \in I} M_{i}=\left[\bigvee_{\varphi \in \bigcup_{i} M_{i}} \varphi\right]$
} 
Many locales are fruitfully described as the classifying locale of a certain propositional geometric theory. For instance:

(1) The localic real line is the classifying locale of the theory of Dedekind cuts. Its points are the models of that theory, that is, the Dedekind reals, and the induced topology on the set of points coincides with the usual Euclidean topology on the reals.

The localic open unit interval is the classifying locale of the same theory but with the additional axiom $\top \vdash \alpha_{0} \wedge \beta_{1}$.

(2) The locale of surjections $\mathbb{N} \rightarrow \mathbb{R}$ is the classifying locale of the theory of surjections $\mathbb{N} \rightarrow \mathbb{R}$.

(3) The spectrum of a ring $A$ is the classifying locale of the theory of prime filters of $A$. The topological space of points of this locale is the Zariski spectrum as familiar from algebraic geometry $n$

(4) The localic Galois group of a field extension $L \mid k$ is the classifying locale of the theory of ring automorphisms $L \rightarrow L$ which fix $k$.

(5) The empty locale is the classifying locale of the inconsistent theory.

(6) The one-point locale is the classifying locale of the empty theory.

Just as we often refer to a topological space only by its points ("the topological space of prime filters of $A$ "), not mentioning its topology, it is customary to abbreviate "the classifying locale of the propositional geometric theory of prime filters of $A$ " as "the locale of prime filters of $A$ ".

Remark 15. The traditional way of constructing the real line or the Zariski spectrum as a topological space proceeds in three steps: (1) Write down the axioms (of Dedekind cuts or of prime filters, respectively). (2) Using the powerset axiom and separation, construct the set of Dedekind reals or the set of prime filters, respectively. (3) Devise a useful topology on the resulting set.

Locales provide us with a more economic and also more conceptual way of arriving at these topological spaces: They can be obtained as the topological space of points of the corresponding classifying locale. In particular, step (3) of manually devising a topology is not necessary when pursuing

\footnotetext{
${ }^{\mathrm{n}}$ Classically, the spectrum is usually defined as the topological space of prime ideals instead of prime filters. However, classically there is a canonical one-to-one correspondence between prime ideals and prime filters, hence one could just as well use filters instead of ideals in the definition. The classifying locale of prime ideals of $A$ also exists, and has the prime ideals of $A$ as its points, but the induced topology on its topological space of points is the flat topology or co-Zariski topology 62 instead of the ordinary Zariski topology 63, Proposition 4.5].
} 
the localic route.

In general, different theories can give rise to isomorphic classifying locales; such theories are called Morita-equivalent. Olivia Caramello has built a vast research program on this observation [59.

Conversely, any locale $X$ is the classifying locale of a certain theory, namely the theory of points of $X$. This theory has one nullary relation symbol $\varphi_{U}$ for each open $U \in \mathcal{O}(X)$ (read as "the point belongs to $U$ ") and the following axioms:

$$
\begin{array}{rlr}
\varphi_{U} & \vdash \varphi_{V} & \text { (for all } U, V \in \mathcal{O}(X) \text { such that } U \preceq V \text { ) } \\
\top & \vdash \varphi_{\top} & \text { (for all } U, V \in \mathcal{O}(X)) \\
\varphi_{U} \wedge \varphi_{V} & \vdash \varphi_{U \wedge V} \\
\varphi_{\bigvee_{i} U_{i}} & \vdash \bigvee_{i} \varphi_{U_{i}} & \text { (for each set-indexed family }\left(U_{i}\right)_{i} \text { of opens) }
\end{array}
$$

These are exactly the axioms for a completely prime filter of $\mathcal{O}(X)$. The isomorphism from $\mathcal{O}(X)$ to the Lindenbaum algebra of this theory maps an open $U$ to $\left[\varphi_{U}\right]$.

The question whether a given locale is spatial is intimately related with the existence of appropriate ideal objects whose existence typically hinges on the axiom of choice or one of its variants. For instance, there are the following results (where we allow ourselves a modicum of classical logic in order to state the results in a familiar form).

(1) The localic spectrum of a ring $A$ is spatial iff every nontrivial ideal (of a certain class of rings related with $A$ ) is contained in a prime ideal.

(2) The localic Galois group of a Galois extension $L \mid k$ is spatial iff any $k$ homomorphism $\sigma: E \rightarrow L$ defined on a finite intermediate extension $L|E| k$ admits an extension to a $k$-homomorphism $L \rightarrow L$.

(3) The localic real line is spatial iff every open covering of the metric space $[0,1]$ of Dedekind reals has a Lebesgue number (that is, if the metric space $[0,1]$ is Heine-Borel compact).

(4) The statement that the classifying locale of any propositional coherent theory (that is, a propositional geometric theory in which only finite disjunctions occur) is spatial is equivalent to the Boolean Prime Ideal Theorem BPIT.

In this picture, the opens of a locale can be regarded as finite approximations to the ideal points which potentially belong to them. For instance, the open $\left[\sigma_{\sqrt{2},-\sqrt{2}} \wedge \sigma_{\sqrt{3}, \sqrt{3}}\right]$ of the localic Galois group of $\overline{\mathbb{Q}} \mid \mathbb{Q}$ can 
be regarded as an approximation to a certain automorphism of $\overline{\mathbb{Q}}$ about which $\sqrt{2} \mapsto-\sqrt{2}$ and $\sqrt{3} \mapsto \sqrt{3}$ is known. Over time, this approximation might be refined to a smaller open, one which contains more information about the purported automorphism, and eventually we might actually obtain an automorphism which is defined on all of $\overline{\mathbb{Q}}$. The opens make good constructive sense even if the points remain elusive.

The results mentioned above explain why we can, in classical mathematics, where all of the stated conditions are satisfied, blithely and fruitfully use the topological spectrum of a ring, the topological Galois group or the topological space of reals. In classical mathematics, employing their localic counterparts is merely for convenience or for aesthetic reasons; in constructive mathematics, localic replacement (or similar replacements in other pointfree approaches to topology) is vital.

Remark 16. The case of the locale of surjections $\mathbb{N} \rightarrow \mathbb{R}$ is particular in that there, even the strong existence axioms of classical mathematics do not suffice to materialize a point. In fact, already the law of excluded middle or the countable axiom of choice preclude the existence of points.

\subsection{Sheaves on locales}

Definition 17. A presheaf $F$ on a locale $X$ is a functor $\mathcal{O}(X)^{\mathrm{op}} \rightarrow$ Set, that is

(1) a set $F(U)$ for each open $U \in \mathcal{O}(X)$ and

(2) a map $\left.(\cdot)\right|_{V} ^{U}: F(U) \rightarrow F(V)$ for each pair of opens $V \preceq U$

such that $\left.(\cdot)\right|_{U} ^{U}=\operatorname{id}_{F(U)}$ for all $U \in \mathcal{O}(X)$ and $\left.\left.(\cdot)\right|_{W} ^{V} \circ(\cdot)\right|_{V} ^{U}=\left.(\cdot)\right|_{W} ^{U}$ for all $W \preceq V \preceq U$.

Definition 17, as well as any other definition in this section, also makes sense for topological spaces instead of locales, since only the notion of opens and their inclusion relation is used. The elements of $F(U)$ are called sections of $F$ over $U$ and the maps $\left.(\cdot)\right|_{V} ^{U}$ are called restriction maps. Elements of $F(T)$ are called global sections.

When first learning about presheaves, one may feel intimidated by the vast amount of data encoded in a single presheaf (one set for each open, of which there are often uncountably many). The situation should be compared to the perhaps more familiar Kripke models for a first-order language $L$. The preorder $W$ of nodes of such a model is like the frame of 
opens of a locale, and the family $\left(M_{w}\right)_{w \in W}$ of $L$-structures, one for each node, is like a presheaf. In fact, the notion of a locale can be generalized to that of a site so that both locales and Kripke frames induce sites and that presheaves on sites can be defined; however, this shall not be pursued here, a textbook reference is Ref. [16.

The prototypical example of a presheaf (and also a sheaf) is the presheaf $\mathcal{C}$ of continuous real-valued functions on a topological space $X$. For this presheaf, the set $\mathcal{C}(U)$ is the set of continuous real-valued maps $U \rightarrow \mathbb{R}$, and the maps $\left.(\cdot)\right|_{V} ^{U}$ are given by actual restriction of functions to smaller domains, that is by

$$
\mathcal{C}(U) \longrightarrow \mathcal{C}(V),\left.s \longmapsto s\right|_{V} .
$$

Any set $M$ gives rise to a presheaf $\underline{M}^{\text {pre }}$, by setting $\underline{M}^{\text {pre }}(U)=M$ for every open $U$ and employing the identity map as restriction maps. In this way, the universe of sets embeds into the universe of presheaves.

\section{Definition 18.}

(1) A compatible family of a presheaf $F$ with respect to an open covering $U=\bigvee_{i} U_{i}$ is a family $\left(s_{i}\right)_{i}$ of sections $s_{i} \in F\left(U_{i}\right)$ such that $\left.s_{i}\right|_{U_{i} \wedge U_{j}} ^{U_{i}}=\left.s_{j}\right|_{U_{i} \wedge U_{j}} ^{U_{j}}$ for all indices $i, j$.

(2) A presheaf $F$ is a sheaf iff for any compatible family $\left(s_{i}\right)_{i}$ with respect to any open covering $U=\bigvee_{i} U_{i}$ there is a unique section $s \in F(U)$ such that $\left.s\right|_{U_{i}} ^{U}=s_{i}$ for all indices $i$ ("compatible sections glue").

For instance, the presheaf $\mathcal{C}$ of continuous real-valued functions is a sheaf, while its subpresheaf $\mathcal{C}_{\mathrm{c}}$ of constant real-valued functions is usually not a sheaf. For instance, on $X=\mathbb{R}$, the two constant functions $f$ : $(-1,0) \rightarrow \mathbb{R}, x \mapsto-1$ and $g:(0,1) \rightarrow \mathbb{R}, x \mapsto 1$ agree on the intersection of their domains (which is empty), but there is no constant function $h$ : $(-1,0) \cup(0,1) \rightarrow \mathbb{R}$ which restricts to $f$ on $(-1,0)$ and to $g$ on $(0,1)$.

There is a general construction called sheafification which turns presheaves into sheaves. Applied to the presheaf $\mathcal{C}_{\mathrm{c}}$, this process yields the sheaf of locally constant real-valued functions. Similarly, the sheafification of the presheaf of bounded functions is the sheaf of locally bounded functions, and so on; this is a general principle.

The presheaf $\underline{M}^{\text {pre }}$ associated to a set $M$ is almost never a sheaf. Its sheafification $\underline{M}$ is the constant sheaf associated to $M$. On an open $U$, its sections are the locale morphisms $U \rightarrow M$, where $M$ is regarded as the locale induced by the discrete topological space $M$ and $U$ is regarded as 
a sublocale of $X$. By the constant sheaf construction, the universe of sets maps to the universe of sheaves on $X$. We will not require much more details on this construction and only note two special cases:

(1) If the locale $X$ is induced by a topological space $Y$, then

$$
\underline{M}(U)=\{f: U \rightarrow M \mid f \text { is locally constant }\} .
$$

(2) If $X$ is the one-point locale, then $\underline{M}(U)=M^{U}$.

In the other direction, given a point $x$ of a locale $X$, any sheaf $F$ on $X$ gives rise to a set, namely the stalk of $F$ at $x$. The stalk is the set of germs of sections of $F$ at $x$, that is the set of all sections of $F$ on opens $U$ such that $x \notin U$, where two such sections $\langle U, s\rangle$ and $\left\langle U^{\prime}, s^{\prime}\right\rangle$ are identified iff there is an open $V \preceq U \wedge U^{\prime}$ such that $x \notin V$ and $\left.s\right|_{V} ^{U}=\left.s^{\prime}\right|_{V} ^{U^{\prime}}$.

For instance, the stalk at the origin of the sheaf of holomorphic functions on $\mathbb{C}$ is in canonical bijection with the set $\mathbb{C}\{z\}$ of convergent power series, and the stalks of a constant sheaf $\underline{M}$ are all in canonical bijection with $M$.

Definition 19. A subsheaf of a sheaf $F$ on a locale $X$ is a sheaf $F^{\prime}$ with $F^{\prime}(U) \subseteq F(U)$ for all opens $U$ and the inherited restriction maps.

Definition 20. A morphism of presheaves $\eta: F \rightarrow G$ on a locale $X$ is a family $\left(\eta_{U}\right)_{U \in \mathcal{O}(X)}$ of maps $\eta_{U}: F(U) \rightarrow G(U)$ such that for all sections $s \in$ $F(U)$ and all opens $V \preceq U$ of $X,\left.\eta_{U}(s)\right|_{V} ^{U}=\eta_{V}\left(\left.s\right|_{V} ^{U}\right)$. A morphism of sheaves is a morphism of the underlying presheaves.

\subsection{Sheaf semantics}

Motivating the sheaf semantics. Let $F$ be a sheaf on a locale $X$. What is a sensible reading of the statement " $\forall s: F .(\varphi(s) \Rightarrow \psi(s))$ "? A first answer is "for any global section $s \in F(X)$ such that $\varphi(s), \psi(s)$ ". However, this proposal does not do justice to the sheaf, whose interesting behavior may well only play out with its sections on small opens. Indeed, many important sheaves have no or few global sections. Hence a more sophisticated answer is "for any open $U \in \mathcal{O}(X)$ and any section $s \in F(U)$ with $\varphi(s), \psi(s)$ ", where " $\varphi(s)$ " and " $\psi(s)$ " have to be interpreted in a similar vein.

Similarly, given a section $s \in F(U)$, what should " $\neg \varphi(s)$ " mean? Since a section may well validate additional properties when we zoom in to smaller opens, the sheaf-theoretically sensible answer is "except for the empty open $V=\perp,\left.s\right|_{V} ^{U}$ does not have property $\varphi$ for any open $V \preceq U$ ", where again " $\varphi\left(\left.s\right|_{V} ^{U}\right)$ " has to be understood in a sensible way. 
As a final motivating example, consider the statement " $\exists s: F . \varphi(s)$ ". This should mean that there is an open covering $X=\bigvee_{i} U_{i}$ such that on each open $U_{i}$, there is a section $s \in F\left(U_{i}\right)$ such that $\varphi(s)$, properly interpreted. We should not require these individual sections to agree on the intersections $U_{i} \wedge U_{j}$ - by the sheaf property, this would amount to saying that there is a global section $s \in F(X)$ with $\varphi(s)$.

The sheaf semantics formalizes the informal language agreement just outlined. A marvelous fact with wide-ranging applications is that this semantics is sound with respect to first-order intuitionistic logic (and even infinitary higher-order intuitionistic logic, and even a suitable version of extensional type theory, if the semantics is appropriately extended).

That is, if we have established a property $\varphi$ and if there is an intuitionistic proof that $\varphi$ entails a further property $\psi$, then we may conclude that also the interpretation of $\psi$ with the sheaf semantics holds.

Hence the sheaf semantics allows us to reason with sheaves in much the same way as we reason with sets, in a simple element-based language, and to import much of the already-existing results in constructive mathematics into the world of sheaves. For instance, any intuitionistic theorem about rings yields a corresponding theorem about sheaves of rings.

There is only one caveat to keep in mind: Irrespective of its status in the metatheory, the interpretation of the law of excluded middle with the sheaf semantics is, for most locales $X$, false (Example 211). Practitioners of other flavors of constructive mathematics should also observe that the interpretation of any form of the axiom of choice, even countable choice or weak countable choice, is false for most locales $X$. (About the only exception are locales induced from discrete topological spaces. For such locales, the law of excluded middle and countable, dependent or full choice pass from the metatheory to the universe of sheaves.)

Incidentally, that the sheaf semantics is not sound with respect to classical logic allows us to illustrate the failure of certain classical theorems in constructive mathematics by geometric counterexamples and thereby verify their unprovability in intuitionistic logic.

We now begin the formal development of the sheaf semantics. More background and examples from theoretical computer science, analysis and differential geometry are contained in Ref. 64, which should be regarded as a companion paper to this contribution. We also recommend Christopher Mulvey's tutorial [65, which culminates in a proof of the Serre-Swan theorem using the sheaf semantics. Standard references for the sheaf semantics include Refs. [16, Chapter VI], 58, Chapter 14], 66, Section 5], 67, Sec- 
tions 12-15], [68, Chapter 6], [12, Part D] and [69, which also gives a detailed account of useful type theory.

Definition and basic properties of the sheaf semantics. Let $X$ be a locale. A formula over an open $U \in \mathcal{O}(X)$ is an infinitary first-order formula (made up using $=\top \wedge \wedge \perp \vee \vee \Rightarrow \forall \exists$ ) over the signature which has one sort for each sheaf $F$, one constant symbol of sort $F$ for each section $s \in F(U)$, one function symbol $f: F \rightarrow G$ for each morphism of sheaves, and so on. As usual, we define $\neg \varphi$ to be an abbreviation of $(\varphi \Rightarrow$ $\perp)$.

Table 5 defines by recursion on the structure of formulas what it means for a formula $\varphi$ over an open $U$ to be forced by $U$ or to be true over $U$, written " $U \models \varphi$ ". The main differences with the perhaps more familiar Kripke semantics are marked. The sheaf semantics is also called KripkeJoyal semantics.

For the special case that $U$ is the largest open of $X$ we also write " $\operatorname{Sh}(X) \models \varphi$ " or " $X \models \varphi$ ".

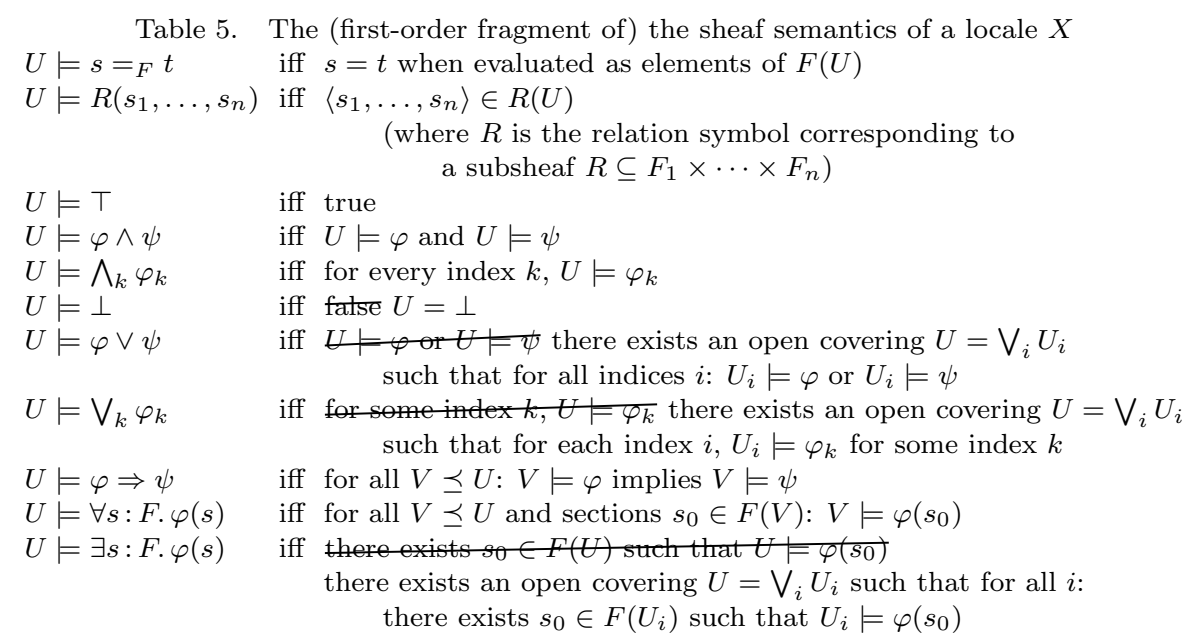

Some clauses in Table 5 contain slight abuses of notation. Namely, if $\varphi$ is a formula over an open $U$ and if $V$ is an open such that $V \preceq U$, then " $V \models \varphi$ " is formally not well-defined, as $\varphi$ is not a formula over $V$. When we still write " $V \models \varphi$ ", we mean that any constant symbol $s \in F(U)$ appearing in $\varphi$ should be interpreted as its restriction $\left.s\right|_{V} ^{U} \in F(V)$, so that 
the resulting formula is a formula over $V$.

Example 21. For instance, let $F$ be the sheaf

$U \longmapsto\left\{f: U \rightarrow \mathbb{C} \mid f\right.$ is continuous and $f(z)^{2}=z$ for all $\left.z \in U\right\}$

on $X=\mathbb{C}$. The interpretation of the statement " $\exists s: F$. $\top$ " is false, as on no open which contains the origin a continuous square root function exists), but the interpretation of " $\neg \neg(\exists s: F . \top)$ " is true: Unrolling the definition, this statement expresses that

the only open $U \preceq X$ which has the property that the only open $V \preceq U$ which has the property that locally on $V$, there exist continuous square root functions is $V=\perp$

is $U=\perp$.

The principal properties of the sheaf semantics is that it is monotone, local and sound with respect to (infinitary) intuitionistic first-order logic as defined in Table 2 on page 21

Theorem 22. Let $X$ be a locale. Let $U$ be an open of $X$. Let $\varphi$ be a formula over $U$.

(1) If $V$ is an open such that $V \preceq U$, then $U \models \varphi$ implies $V \models \varphi$.

(2) Let $U=\bigvee_{i} U_{i}$ be an open covering. If $U_{i} \models \varphi$ for all $i$, then $U \models \varphi$.

(3) Let $\psi$ be a further formula over $U$. If $U \models \varphi$ and if $\varphi$ entails $\psi$ intuitionistically, then also $U \models \psi$.

Proof. The proof of (1) is by a routine induction on the structure of $\varphi$. For the clauses pertaining disjunction and existential quantification, the distributive law has to be used.

The proof of (2) is also a routine induction proof. For the clause pertaining equality, the sheaf condition has to be used.

To verify statement (3), it is prudent to generalize the claim slightly: An induction on the structure of derivations shows that if a sequent $\varphi \vdash_{x_{1}: X_{1}, \ldots, x_{n}: X_{n}} \psi$ is derivable in infinitary intuitionistic first-order logic, then $U \models \forall x_{1}: X_{1} \ldots \forall x_{n}: X_{n} \cdot(\varphi \Rightarrow \psi)$.

Remark 23. The proof of Theorem 22 is constructive. Hence, by unrolling the definition of the sheaf semantics, any proof which uses the sheaf semantics as a simplifying device can mechanically be unwound to a fully explicit proof which does not reference sheaves or the sheaf semantics. 
In general, the resulting proofs will be longer and less perspicuous than the original proofs, in particular if the original proofs employed nested implications (such as double negations). In these cases, there is a certain "exotic flavor" to the unwound proofs, as with tricks with continuations in computer science. We display some such proofs which are still readable in Section 3.5

Sheaf models. Definition 24. A sheaf model of a geometric theory $\mathbb{T}$ over a locale $X$ consists of

(1) a sheaf $\llbracket S \rrbracket$ on $X$ for each sort $S$,

(2) a morphism $\llbracket f \rrbracket: \llbracket S_{1} \rrbracket \times \cdots \times \llbracket S_{n} \rrbracket \rightarrow \llbracket T \rrbracket$ of sheaves for each function symbol $f: S_{1} \times \cdots \times S_{n} \rightarrow T$ and

(3) a subsheaf $\llbracket R \rrbracket \subseteq \llbracket S_{1} \rrbracket \times \cdots \times \llbracket S_{n} \rrbracket$ for each relation symbol $R \hookrightarrow$ $S_{1} \times \cdots \times S_{n}$

such that the axioms of $\mathbb{T}$ are validated under the sheaf semantics.

Example 25. The sheaf $\mathcal{C}$ of continuous real-valued functions on any locale $X$ together with appropriate morphisms is a sheaf model of the theory of rings. For instance, the interpretation of the axiom

$$
\forall f: \mathcal{C} . \forall g: \mathcal{C} . f+g=g+f
$$

with the sheaf semantics amounts to the condition

For any open $U$ and any continuous function $f \in \mathcal{C}(U)$,

for any open $V$ and any continuous function $g \in \mathcal{C}(V)$,

$$
\left.f\right|_{V} ^{U}+g=g+\left.f\right|_{V} ^{U} .
$$

and is readily seen to be fulfilled.

Example 26. Any sheaf on any locale can be regarded as a sheaf model of the theory of objects.

Example 27. Any set-based model $M$ of a geometric theory $\mathbb{T}$ gives rise to a sheaf model over the one-point locale, by employing the constant sheaf construction reviewed on page 33. An infinitary first-order formula holds for $M$ iff it holds for the induced sheaf model. Hence sheaf models are strictly more general than set-based models.

In the special case of nullary function symbols or relations symbols,

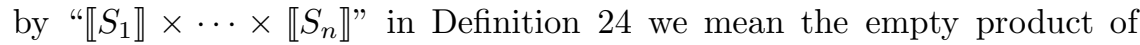
sheaves, the terminal sheaf 1 with $1(U)=\{\star\}$ for every open $U$ of $X$. 
Morphisms $1 \rightarrow F$ are in canonical bijection with global sections of $F$. Subsheaves $F \subseteq 1$ are in canonical bijection with the opens of $X$ (Exercise 25).

With this in mind, we can rephrase Definition 24 in the special case of propositional theories in the following simpler form. A sheaf model of a propositional geometric theory $\mathbb{T}$ over a locale $X$ consists of a family $\left(U_{\varphi}\right)_{\varphi}$ of opens of $X$, one open for each nullary relation symbol $\varphi$ of $\mathbb{T}$, in such a way that the axioms are satisfied. For instance, that an axiom " $\alpha \wedge \beta \vdash$ $\bigvee_{i} \gamma_{i}$ " is satisfied means that $U_{\alpha} \wedge U_{\beta} \preceq \bigvee_{i} U_{\gamma_{i}}$.

In other words, a model of a propositional geometric theory $\mathbb{T}$ over a locale $X$ is the same as a frame homomorphism $\mathcal{O}(L(\mathbb{T})) \rightarrow \mathcal{O}(X)$, hence a locale morphism $X \rightarrow L(\mathbb{T})$.

For further reference we state a proposition relating truth in a sheaf model with truth at all points.

Proposition 28. Let $\mathbb{T}$ be a geometric theory without any axioms. Let $M$ be a sheaf model of $\mathbb{T}$ on a locale $X$ (hence just a sheaf structure) and consider the structures $M_{x}$ obtained by computing the stalks at points $x \in$ $\operatorname{Pt}(X)$. Let $\sigma$ be a geometric sequent. Then:

(1) If $M$ validates $\sigma$, then so does $M_{x}$ for any point $x \in \operatorname{Pt}(X)$.

(2) The converse holds if $X$ is spatial.

Proof. See [12, Corollary D1.2.14(ii)].

In particular, the stalks of sheaf models of a geometric theory $\mathbb{T}$ are set-based models of $\mathbb{T}$.

The generic model. A geometric theory can well have no set-based models, but might still be consistent and allow for a diverse range of sheaf models on a host of different locales. In fact, any propositional geometric theory always has at least one, and very peculiar, sheaf model, namely its generic model.

Definition 29. The generic model $U_{\mathbb{T}}$ of a propositional geometric theory $\mathbb{T}$ is the sheaf model on $L(\mathbb{T})$ given by $U_{\varphi}:=[\varphi]$ for all nullary relation symbols $\varphi$.

Under the view of models as locale morphisms, the generic model $U_{\mathbb{T}}$ corresponds to the identity morphism $L(\mathbb{T}) \rightarrow L(\mathbb{T})$. If a $\mathbb{T}$-model $M$ on a 
locale $X$ is given by a locale morphism $f: X \rightarrow L(\mathbb{T})$, we also say that " $M$ arises from $U_{\mathbb{T}}$ by pullback along $f^{\prime \prime}$.

For instance, there is the generic Dedekind cut, the generic model of the theory $\mathbb{T}$ of Dedekind cuts on the classifying locale of $\mathbb{T}$, the localic real line. By Example 27, any specific Dedekind real $x$ (that is, any point $x$ of $L(\mathbb{T})$ ) gives rise to a model of $\mathbb{T}$ on the one-point locale pt, corresponding to the locale morphism $x: \mathrm{pt} \rightarrow L(\mathbb{T})$.

For a geometric sequent $\sigma$ over the signature of a geometric theory $\mathbb{T}$, the following statements are equivalent:

(1) The generic model of $\mathbb{T}$ validates $\sigma$.

(2) All sheaf models of $\mathbb{T}$ on arbitrary locales validate $\sigma$.

(3) The theory $\mathbb{T}$ proves $\sigma$.

Hence we may understand the phrase "Let $M$ be a model of $\mathbb{T}$." to implicitly refer to the generic model. The first-order properties of the generic model need not be shared by all sheaf models and need not be provable by $\mathbb{T}$.

The story of generic models is at the same time tautologous and deep, and these short paragraphs do not do justice to this intriguing aspect of the interplay between topology (in the form of locales) and logic (in the form of propositional geometric theories). We strongly recommend the accessible account by Steve Vickers [5] for a fuller picture.

Remark 30. The traditional way of constructing a map of topological spaces $f: Y \rightarrow Y^{\prime}$ proceeds in three steps: (1) Write down a map of the underlying sets. (2) Identify the preimages $f^{-1}[U]$. (3) Verify that these preimages are open.

Continuing Remark 15, locales provide more efficient means for constructing morphisms: To write down a morphism of locales $X \rightarrow X^{\prime}$, where $X^{\prime}$ is given as the classifying locale of some theory $\mathbb{T}^{\prime}$, we only need to specify a $\mathbb{T}^{\prime}$-model $M^{\prime}$ on $X$; no separate verification of continuity is required.

If $X$ classifies a theory $\mathbb{T}$, such a specification is usually carried out by constructing $M^{\prime}$ in terms of the generic model $U_{\mathbb{T}}$. For instance, if $f$ : $A \rightarrow B$ is a ring homomorphism, we obtain a locale morphism $\operatorname{Spec}(B) \rightarrow$ $\operatorname{Spec}(A)$ by specifying the model $([D(f(x))])_{x \in A}$ of the theory of prime filters of $A$ on $\operatorname{Spec}(B)$. On the level of points, this morphism induces the expected map $\mathfrak{f} \mapsto f^{-1}[\mathfrak{f}]$. 


\section{Exercises}

Exercise 14 (The theory of finite objects). (a) Extend the geometric theory of objects (page 22) by relation symbols and axioms, but not by constant symbols, to ensure that the set-based models are precisely the two-element sets.

(b) Write down a geometric theory whose set-based models are precisely the (Kuratowski-)finite sets.

Hint. Employ countably many relation symbols $R_{n} \hookrightarrow X \times \cdots \times X$, where $R_{n}\left(x_{1}, \ldots, x_{n}\right)$ should signify that the elements $x_{1}, \ldots, x_{n}$ exhaust all elements of $X$. A solution to this exercise is given in Ref. [12 Example D1.1.7(k)].

Exercise 15 (Issues of size). Working in IZF, show that the Lindenbaum algebra of a propositional geometric theory $\mathbb{T}$ can be realized as a set, even though the class of geometric formulas is typically a proper class. Hint. Use that any geometric formula is provably equivalent to one of the form $\bigvee_{i} \varphi_{i}$, where each formula $\varphi_{i}$ is a conjunction of nullary relation symbols.

Exercise 16 (Discrete locales). (a) Find a theory which is classified by the two-point locale (the locale associated to the discrete two-point topological space).

(b) Let $M$ be a set and endow it with the discrete topology. Find a theory which is classified by $L(M)$.

Exercise 17 (The localic unit interval). Extend the theory of Dedekind cuts in such a way that its models are those Dedekind reals which are elements of $[0,1]$.

Exercise 18 (More on exotic locales). (a) Define the locale of partial surjections $\mathbb{N} \rightarrow \mathbb{N}^{\mathbb{N}}$.

Note. In Russian constructivism, as for instance reigning in the effective topos [70 72], the Baire space $\mathbb{N}^{\mathbb{N}}$ is subcountable. However, in this setting any function $\mathbb{N} \rightarrow \mathbb{N}$ is computable. The universe of sheaves over this locale was the first example of a setting in which the Baire space is subcountable and still any external number-theoretic function can be imported [73, Section 4.3].

(b) Define the locale of injections $\mathbb{R} \hookrightarrow \mathbb{N}$.

Note. In classical mathematics, this locale does not have any points. However there is a variant of the effective topos based on the infinite-time Turing machines by Joel David Hamkins and Andy Lewis 74 in which an injection $\mathbb{R} \hookrightarrow \mathbb{N}$ exists 75. See Ref. 64 Section 2.2] for an exposition of the proof.

(c) Define the locale of "open covers of $[0,1]$ by intervals with rational endpoints such that the union of any finite number of those intervals has total Lebesgue measure less than $\frac{1}{2}$ ".

Note. In the universe of sheaves over this locale, the set of unit interval Dedekind real numbers fails to be (Heine-Borel) compact and the localic real line fails to be spatial [12] Example D4.7.13]. 
(d) An open $U$ of a locale $X$ is complemented iff there is an open $V$ such that $U \vee V=\top$ and $U \wedge V=\perp$. A locale is zero-dimensional iff any open is a join of complemented opens. Assuming that any two reals are equal or not, show that the locale of surjections $\mathbb{N} \rightarrow \mathbb{R}$ is zero-dimensional.

Exercise 19 (Models as points). Let $\mathbb{T}$ be a propositional geometric theory. Verify that the points of $L(\mathbb{T})$ are in canonical bijection with the set-based models of $\mathbb{T}$.

Exercise 20 (The product of locales). Let $X$ and $X^{\prime}$ be locales, classifying propositional geometric theories $\mathbb{T}$ and $\mathbb{T}^{\prime}$ respectively. Let $\mathbb{T}+\mathbb{T}^{\prime}$ be the combined theory (whose set of sorts is the disjoint union of the sets of sorts of $\mathbb{T}$ and $\mathbb{T}^{\prime}$, and so on). Show that $L\left(\mathbb{T}+\mathbb{T}^{\prime}\right)$ is the product of $X$ and $X^{\prime}$ in the category of locales.

Exercise 21 (The coproduct of locales, revisited). Let $X$ and $X^{\prime}$ be locales, classifying some propositional geometric theories $\mathbb{T}$ and $\mathbb{T}^{\prime}$ respectively. Find a theory in terms of $\mathbb{T}$ and $\mathbb{T}^{\prime}$ which is classified by $X \amalg X^{\prime}$.

Exercise 22 (Spatiality of the localic reals). Let $\mathbb{R}$ be the localic real line, defined as the classifying locale of the theory $\mathbb{T}$ of Dedekind cuts as introduced on page 23, For rational numbers $x, y$, let "( $(x, y)$ " denote the formula $\alpha_{x} \wedge \beta_{y}$. Let $(x, y)$ denote the open interval $\{\xi \in \operatorname{Pt}(\mathbb{R}) \mid x<\xi<y\}$ in the set of Dedekind reals.

(a) Extend the notation to allow for $x, y= \pm \infty$.

(b) Show that the canonical topology on $\operatorname{Pt}(\mathbb{R})$, defined in Exercise 1 coincides with the usual Euclidean topology.

(c) Show that any open of $\mathbb{R}$ can be written in the form $\bigvee_{i}\left[\left(x_{i}, y_{i} \cdot\right)\right]$ with $x_{i}, y_{i} \in \mathbb{Q} \cup\{ \pm \infty\}$.

(d) Let rational numbers be given such that $(x, y) \subseteq \bigcup_{i}\left(x_{i}, y_{i}\right)$ in $\operatorname{Pt}(\mathbb{R})$. Assume that this open covering has a Lebesgue number. Show that $\mathbb{T}$ proves $(\cdot x, y \cdot) \vdash \bigvee_{i}\left(\cdot x_{i}, y_{i}\right)$.

(e) Assume that closed intervals in the set of Dedekind reals are (HeineBorel) compact. Then redo part (d), but without the assumption on the existence of a Lebesgue number.

(f) Under the same assumption as in part (e), show that the localic real line is spatial.

Note. Exercise 18 lists settings in which the assumption in part (e) is not satisfied. The converse in part (f) also holds. Incidentally, the spatiality of the localic real line is an example 
for a statement which requires the full version of the Heine-Borel property, not only HeineBorel for countable covers as it is often studied in reverse mathematics [76].

Exercise 23 (The localic Galois group of a field extension). Let $L \mid k$ be a field extension. The localic Galois group $\operatorname{Gal}(L \mid k)$ is the classifying locale of the theory of automorphisms $L \rightarrow L$ which fix $k$, as described on page 24 .

(a) Explain how to obtain a morphism $\operatorname{Gal}(L \mid k) \rightarrow \operatorname{Gal}(L \mid k)$ which on the level of points should send an automorphism $\sigma$ to its inverse $\sigma^{-1}$.

(b) Explain how to obtain a morphism $\operatorname{Gal}(L \mid k) \times \operatorname{Gal}(L \mid k) \rightarrow \operatorname{Gal}(L \mid k)$ which on the level of points should send a pair $\langle\sigma, \tau\rangle$ to $\sigma \circ \tau$.

(c) Assume from now on that $L$ is a geometric field in the sense that any element is zero or invertible. Show that the axiom expressing injectivity can be dropped without changing the set of provable sequents.

(d) Let $f \in k[X]$ be a monic polynomial such that $f(X)=\left(X-x_{1}\right) \cdots(X-$ $x_{n}$ ) over $L$. Let $x$ be one of the zeros of $f$. Show that the theory proves $\top \vdash \bigvee_{i=1}^{n} \sigma_{x, x_{i}}$.

(e) Now furthermore assume that $L$ is Galois over $k$ in the sense that any element of $L$ is the zero of a monic polynomial $f \in k[X]$ which is separable (that is, $\left.\left(f, f^{\prime}\right)=(1) \subseteq k[X]\right)$ and splits over $L$ into linear factors. Show that the axioms expressing surjectivity can be dropped.

(f) Show that the topological space of points of $\operatorname{Gal}(L \mid k)$ is canonically homeomorphic to the topological Galois group of $L \mid k$.

(g) Show that $\operatorname{Gal}(L \mid k)$ is spatial in classical mathematics.

Exercise 24 (Coherent locales and coherent theories). An open $U$ of a locale is compact if and only if, whenever $U \preceq \bigvee_{i \in I} V_{i}$, there is a (Kuratowski-)finite subset $I^{\prime} \subseteq I$ such that $U \preceq \bigvee_{i \in I^{\prime}} V_{i}$. A locale is coherent iff its compact opens are closed under finite meets and if every open is a join of compact opens. A geometric formula is coherent iff only finite disjunctions occur in it. A geometric theory is coherent iff all of its axioms consist of coherent formulas.

(a) Show that any coherent locale classifies some coherent theory.

(b) Let $\mathbb{T}$ be a propositional coherent theory. Show that $L(\mathbb{T})$ is coherent.

Hint. Proceed similarly as in the proof of Theorem 11 For coherent formulas $\alpha$ and geometric formulas $\varphi$, define a semantics by the clauses below. Show by an induction on the structure of geometric derivations that, if $\mathbb{T}$ proves a geometric sequent $\varphi \vdash \psi$, then for all coherent formulas $\alpha$ such that $\alpha \models \varphi, \alpha \models \psi$. Show for coherent formulas $\alpha, \beta$ that $\alpha \models \beta$ iff $\mathbb{T}$ proves $\alpha \vdash \beta$. Use these ingredients to show that, if $\alpha$ is a coherent formula and $\left(\beta_{i}\right)_{i \in I}$ is a family of coherent formulas, if $\mathbb{T}$ proves $\alpha \vdash \bigvee_{i \in I} \beta_{i}$ then there is a (Kuratowski-)finite subset $I^{\prime} \subseteq I$ such that $\mathbb{T}$ proves $\alpha \vdash \bigvee_{i \in I^{\prime}} \beta_{i}$. 


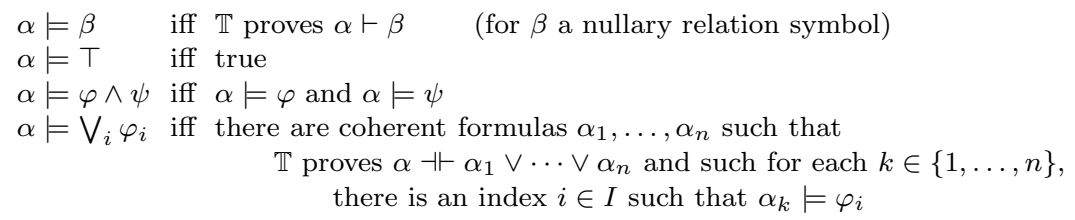

(c) Show that an arbitrary (set-indexed) product of coherent locales is coherent.

(d) Deligne's completeness theorem states that coherent locales (and even coherent toposes) are spatial. Show that Deligne's result implies that propositional coherent theories without any set-based models are inconsistent. (This is usually phrased as "consistent theories have models".)

Exercise 25 (Subsheaves of the terminal sheaf). Let $X$ be a locale.

(a) Show that any subsheaf $F \subseteq 1$ of the terminal sheaf on $X$ gives rise to an open of $X$ by gluing all its sections.

(b) Show that the construction of part (a) yields a canonical bijection of the subsheaves of 1 and the opens of $X$.

Exercise 26 (A geometric interpretation of double negation). Let $\varphi$ be a formula over an open $U$ of a locale $X$.

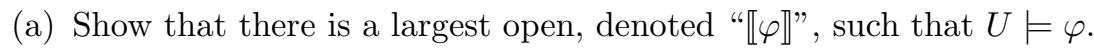
Hint. Consider the join of all opens $V$ such that $V \models \varphi$.

(b) Show that $X \models \neg \neg \varphi$ iff $\llbracket \varphi \rrbracket$ is dense in the sense of Exercise 8 .

(c) Give a condition on a locale or a topological space such that its sheaf semantics validates the law of excluded middle (or equivalently the law of double negation elimination).

Exercise 27 (Unique local existence is global existence). Let $F$ be a sheaf on a locale $X$. Let $\varphi$ be a formula over $X$. Assume that $\operatorname{Sh}(X) \models$ $\exists ! s: F . \varphi(s)$, that is

$$
\operatorname{Sh}(X) \models \exists s: F . \varphi(s) \quad \text { and } \quad \operatorname{Sh}(X) \models \forall s: F . \forall t: F .(\varphi(s) \wedge \varphi(t) \Rightarrow s=t) .
$$

Show that for any open $U \in \mathcal{O}(X)$, there is a unique section $s \in F(U)$ such that $U \models \varphi(s)$.

Exercise 28 (The sheaf of real functions as a field). Let $X$ be a topological space. Let $\mathcal{C}$ be the sheaf of continuous real-valued functions on $X$. 
(a) Let $U$ be an open of $X$. Let $f \in \mathcal{C}(U)$. Show using the result of Exercise 27 that $U \models(\exists g: \mathcal{C} . f g=\mathcal{C} 1)$ iff there is a function $g \in \mathcal{C}(U)$ such that $f g=1$.

(b) Show that $\mathcal{C}$ is a field in that $X \models \forall f: \mathcal{C} .(\neg(\exists g: \mathcal{C} . f g=1)) \Rightarrow f=0$.

(c) Give an example of space $X$ such that it is not the case that $\mathcal{C}$ is a field in the stronger sense that $\operatorname{Sh}(X) \models \forall f: \mathcal{C} .(\exists g: \mathcal{C} . f g=1) \vee f=0$.

(d) Let $X=\mathbb{C}$ and let $\mathcal{O}$ be the sheaf of holomorphic functions on $X$. Show that, in classical mathematics, the sheaf $\mathcal{O}$ is discrete in that $\operatorname{Sh}(\mathbb{C}) \models \forall f: \mathcal{O} . \forall g: \mathcal{O} . f=g \vee \neg(f=g)$.

Exercise 29 (The failure of the fundamental theorem of algebra). Let $F$ be the sheaf of continuous complex-valued functions on $\mathbb{C}$. Give an example of a function $f: \mathbb{C} \rightarrow \mathbb{C}$ such that it is not the case that

$$
\operatorname{Sh}(\mathbb{C}) \models \exists z: F . z^{2}=f .
$$

Note. The sheaf $F$ can be obtained by constructing, in the universe of sheaves on $\mathbb{C}$, the set of pairs of Dedekind reals. Hence the example demonstrates that the usual formulation of the fundamental theorem of algebra does not admit a constructive proof. (However, there are replacements available [77, and the fundamental theorem does apply to the Cauchy complex numbers 78] and under weak choice principles 79]).

Exercise 30 (The least number principle). (a) A subset $M \subseteq X$ of a set $X$ is detachable iff for any element $x \in X$, either $x \in M$ or $x \notin M$. Show that any inhabited detachable subset $M \subseteq \mathbb{N}$ contains a minimal element.

(b) Show that for any inhabited set $M$ of natural numbers, it is not not the case that there is a number $n \in M$ such that $n \leq m$ for all $m \in M$.

(c) Show that for every finitely generated vector space $V$ over a field in the sense that nonunits are zero, it is not not the case that $V$ has a basis.

(d) Verify that the assumption that any inhabited set of natural numbers contains a minimal element implies the law of excluded middle. Hint. Consider a set of the form $\{n \in \mathbb{N} \mid n=1 \vee \varphi\}$.

(e) Give an explicit example of a subsheaf $F$ of the constant sheaf $\underline{\mathbb{N}}$ on a topological space $X$ such that

$$
\operatorname{Sh}(X) \models \exists n: F . \top \quad \text { but } \quad \operatorname{Sh}(X) \not \models \exists n: F . \forall m: F . n \leq m .
$$

\section{Applications in constructive algebra}

We recall from the introduction one way how locales and sheaves yield new reduction techniques for constructive algebra: 
(1) Instead of replacing a given ring with another ring (for instance a ring $A$ with one of its stalks $A_{\mathfrak{p}}$ at a minimal prime ideal), we replace a given ring with a sheaf of rings.

(2) We then maintain the convenient illusion that we are working with a plain old ring instead of a sheaf by employing the sheaf semantics.

The goal of this section is to give a leisurely introduction to this procedure. We recall in Section 3.1 algebraic preliminaries. Section 3.2 explores the sheaf to use. There is an interesting origin story to this sheaf, which we tell in Section 3.3 For computations, it is vital to have an explicit description of this sheaf available; such a description is presented in Section 3.4 Finally, we illustrate how to use the technique in a series of concrete examples in Section 3.5

\subsection{Algebraic preliminaries}

By ring, we mean commutative ring with unit. We do not require the unit to be distinct from the zero; indeed, trivial rings have intriguing applications [13].

A ring is reduced iff zero is its only nilpotent element; that is, if $x^{n}=0$ implies $x=0$.

An $i d e a l$ is a subset $\mathfrak{a} \subseteq A$ such that $0 \in \mathfrak{a},(x \in \mathfrak{p}) \wedge(y \in \mathfrak{p}) \Rightarrow(x+y \in \mathfrak{p})$ and $(x \in \mathfrak{p}) \Rightarrow(x y \in \mathfrak{p})$ (for any $y \in A)$. A family $\left(x_{i}\right)_{i}$ of ring elements generates an ideal, also denoted " $\left(x_{i}\right)_{i}$ ", consisting of (Kuratowski-finite) sums of multiples of the $x_{i}$.

An ideal $\mathfrak{p}$ is prime iff $1 \notin \mathfrak{p}$ and $(x y \in \mathfrak{p}) \Rightarrow(x \in \mathfrak{p}) \vee(y \in \mathfrak{p})$. A minimal prime ideal is a prime ideal $\mathfrak{p}$ such that for any prime ideal $\mathfrak{q}$ with $\mathfrak{q} \subseteq \mathfrak{p}, \mathfrak{q}=\mathfrak{p}$.

A subset $\mathfrak{f} \subseteq A$ is a prime filter iff $0 \notin \mathfrak{f},(x+y \in \mathfrak{f}) \Rightarrow(x \in \mathfrak{f}) \vee(y \in \mathfrak{f})$, $1 \in \mathfrak{f}$ and $(x y \in \mathfrak{f}) \Leftrightarrow(x \in \mathfrak{f}) \wedge(y \in \mathfrak{f})$.

An ideal $\mathfrak{a} \subseteq A$ is a radical ideal iff $x^{n} \in \mathfrak{a}$ implies $x \in \mathfrak{a}$ for any $x \in A$ and any natural number $n$. The radical $\sqrt{\mathfrak{a}}$ of an arbitrary ideal $\mathfrak{a}$ is the radical ideal $\left\{x \in A \mid \exists n \in \mathbb{N} . x^{n} \in \mathfrak{a}\right\}$.

A ring is local iff a finite sum is invertible only if one of its summands also is. More precisely, this means that $1 \neq 0$ and that whenever $a+b$ is invertible, $a$ is invertible or $b$ is invertible. This definition is an elementary rephrasing of the textbook definition according to which a ring is local iff it has exactly one maximal ideal. The elementary definition works better in constructive contexts.

If $S$ is a multiplicatively closed subset of a ring $A$, the localization $A\left[S^{-1}\right]$ 
is the ring of formal fractions $\frac{a}{s}$ with $a \in A$ and $s \in S$, where two such fractions $\frac{a}{s}$ and $\frac{b}{t}$ are deemed equivalent iff there is an element $u \in S$ such that $u t a=u s b$. We do not require that $0 \notin S$; instead we plainly observe that $A\left[S^{-1}\right]$ is the zero ring iff $0 \in S$. We also remark that $\frac{a}{s}=0$ in $A\left[S^{-1}\right]$ iff $u a=0$ for some element $u \in S$.

An important special case is localizing away from an element: If $f$ is an element of $A$, then $A\left[f^{-1}\right]$ is $A\left[\left\{1, f, f^{2}, f^{3}, \ldots\right\}^{-1}\right]$. This ring is the zero ring iff $f$ is nilpotent.

The stalk at a prime ideal $\mathfrak{p} \subseteq A$ is the localization $A_{\mathfrak{p}}:=A\left[(A \backslash \mathfrak{p})^{-1}\right]$. Assuming de Morgan's laws, such stalks are local rings. The stalk at a prime filter $\mathfrak{f} \subseteq A$ is defined as $A_{\mathfrak{f}}:=A\left[\mathfrak{f}^{-1}\right]$ and is a local ring even constructively. Conversely, if a localization $A\left[S^{-1}\right]$ is local then $S$ can be saturated to a prime filter.

A property of rings is localization-stable iff it is inherited by any localization. For instance, if $A$ is a reduced ring, then so is any localization $A\left[S^{-1}\right]$.

\subsection{A remarkable sheaf}

Let $A$ be a ring. Then there is a certain locale $X$ on which a certain "mirror image" of $A$ exists, a sheaf $A^{\sim}$ of rings. On the one hand, this mirror image is "close to $A$ ", such that studying $A^{\sim}$ pays off for learning about $A$. On the other hand, it has much better properties than $A$ has.

$$
A^{\sim} \text { is close to } A \text { : }
$$

(A) There is a canonical isomorphism $A \rightarrow A^{\sim}(X)$ of rings.

(B) $A^{\sim}$ inherits any property of $A$ which is localization-stable.*

(C) A geometric sequent holds for $A^{\sim}$ iff ${ }^{\star \star}$ it holds for all stalks $A_{\mathfrak{f}}$.

${ }^{\star}$ precise formulation in Proposition $\underline{37}$

*assuming BPIT for the "if" direction

$A^{\sim}$ has better properties than $A$ :

(D) $A^{\sim}$ is a local ring.

Assuming that $A$ is reduced:

(E) $A^{\sim}$ is a field: $\forall x: A^{\sim} .\left(\left(\neg\left(\exists y: A^{\sim} . x y=1\right)\right) \Rightarrow x=0\right)$.

(F) $A^{\sim}$ has $\neg \neg$-stable equality: $\forall x, y: A^{\sim} . \neg \neg(x=y) \Rightarrow x=y$.

(G) $A^{\sim}$ is anonymously Noetherian.

Recall that when we ascribe some ring-theoretic property to $A^{\sim}$, we are 
implicitly using the sheaf semantics. For instance, when we say that $A^{\sim}$ is a local ring, we actually mean

$$
\begin{aligned}
& \operatorname{Sh}(X) \models \neg\left(1={ }_{A \sim 0} 0\right) \text { and } \\
& \operatorname{Sh}(X) \models \forall x, y: A^{\sim} .\left(\left(\exists z: A^{\sim} .(x+y) z={ }_{A^{\sim}} 1\right) \Longrightarrow\right. \\
& \left.\quad\left(\exists z: A^{\sim} \cdot x z={ }_{A^{\sim}} 1\right) \vee\left(\exists z: A^{\sim} . y z={ }_{A^{\sim}} 1\right)\right) .
\end{aligned}
$$

The locale $X$ on which this theater plays out is none other than the spectrum of $A$ from algebraic geometry, and the sheaf $A^{\sim}$ is the sheaf of regular functions, also denoted " $\mathcal{O}_{\operatorname{Spec}(A)}$ ". We will give details on its construction in Section 3.3, but first want to reflect on the displayed statements.

A reification of all stalks. Statement (C) explains that, to a first approximation, the sheaf $A^{\sim}$ is a reification of all the stalks of $A$ into a single coherent entity: The sheaf $A^{\sim}$ has exactly those properties which are shared by all the stalks $A_{\mathfrak{f}}$. For instance, the sheaf $A^{\sim}$ is an integral domain iff all the stalks are.

But crucially, this metatheorem only pertains to properties which can be formulated as geometric sequents. Being an integral domain is such a property $\left(1=0 \vdash \perp, x y=0 \vdash_{x: R, y: R} x=0 \vee y=0\right)$, but many others are not. The reductive power of harnessing $A^{\sim}$ stems from the fact that $A^{\sim}$ enjoys properties which are not shared by $A$, by its stalks or indeed by any of its localizations or quotients.

Back and forth. Statement (A) is a kind of conservativity result. For instance, if we want to show that a certain ring element $x \in A$ is zero, we can just as well show that its mirror image in $A^{\sim}$ is zero.

For this step to be useful, we need to know what $A^{\sim}$ looks like. Statements $(\mathbb{C})$ and $(\mathbb{B})$ both allow us to transfer our knowledge about $A$ to $A^{\sim}$; while statement (C) requires a syntactic restriction on the property under consideration, statement $(\mathbb{B})$ requires a semantic one.

Not only does the ring $A$ have a mirror image as a sheaf, also any $A$ module $M$ does. Its mirror image is the quasicoherent sheaf $M^{\sim}$ from algebraic geometry. As with $A$ and $A^{\sim}$, there is a close connection between $M$ and $M^{\sim}$; for instance, the module $M$ is finitely generated over $A$ iff $M^{\sim}$ is finitely generated over $A^{\sim}$ (Exercise 36 ).

The field property. The fact that $A^{\sim}$ is a field is where $A^{\sim}$ derives its main usefulness as a reduction technique from. While statements (B), 
(C) and (D) are immediate consequences of the way $A^{\sim}$ is constructed (Proposition 37), the field property (E) requires an actual computation for its verification and was a surprising discovery by Christopher Mulvey in the 1970s.

Myles Tierney commented around that time that the field property "is surely important, though its precise significance is still somewhat obscureas is the case with many such nongeometric formulas" [54, p. 209]. Even though the property has been known for a long time, only recently was it put in a wider context (recognizing it as a small shadow of algebraic geometry's quasicoherence) and its usefulness for constructive algebra appreciated $[\mathbf{8 0}$, Sections 3.3 and 11.4].

The field property is a unique feature of $A^{\sim}$; it cannot be mimicked by classical techniques in commutative algebra. Several constructions come close, but fail in other ways:

(1) The stalks $A_{\mathfrak{m}}$ at maximal ideals: These are not fields.

(2) The stalks $A_{\mathfrak{p}}$ at minimal prime ideals: These are fields, but a ring element $x \in A$ which is zero in all these stalks $A_{\mathfrak{p}}$ is not necessarily zero in $A$. Also, properties of the stalks tend to only spread to dense opens of the spectrum, if they do so at all, while properties of $A^{\sim}$ are in close connection with properties of $A$. For instance, that all stalks $M_{\mathfrak{p}}$ of an $A$-module $M$ at minimal (or, for that matter, all) prime ideals are finitely generated does not imply that $M$ is finitely generated, while finite generation of $M^{\sim}$ does imply finite generation of $M$.

(3) The quotients $A / \mathfrak{m}$ : These are fields, but an element $x \in A$ which is zero in all these quotients $A / \mathfrak{m}$ is not necessarily zero in $A$, and passing to $A / \mathfrak{m}$ is not exact (does not preserve injectivity of linear maps).

(4) The quotients $A / \mathfrak{p}$ modulo the prime ideals: These are merely integral domains.

The field condition displayed in statement $(\mathrm{E})$ is not the only field condition used in constructive algebra. Perhaps the most important such condition is that any element is zero or invertible, not least because this condition can be expressed as a geometric formula. It is stronger than the condition that nonunits are zero and is satisfied by $A^{\sim}$ iff $A$ is reduced and of Krull dimension $\leq 0$ [80, Proposition 2.13].

Double negation stability. Generally in constructive mathematics, two elements being not not equal does not imply that they are actually equal: 
(1) Sheaves give counterexamples: The interpretation of the statement that two given sections of a sheaf are not not equal with the sheaf semantics is that they agree on a dense open (Exercise 26). This does not generally imply that they agree on all of their domain.

(2) Realizability gives counterexamples: Realizers of negated statements are always uninformative; in the case that a statement $\varphi$ has a realizer at all, the statement $\neg \neg \varphi$ is realized by any number whatsoever. Only in certain cases, where a realizer can be algorithmically reconstructed from the mere promise that a realizer exists, is $\neg \neg \varphi \Rightarrow \varphi$ realizable.

Hence statement $(\mathbb{E})$ comes at a surprise. It is useful to import some of classical logic into the constructive setting of sheaves on $\operatorname{Spec}(A)$. For instance, the law of excluded middle is constructively valid in the form

$$
\neg \neg(\varphi \vee \neg \varphi) .
$$

Combined with the observation

$$
(\neg \neg \chi \wedge(\chi \Rightarrow \neg \neg \psi)) \Longrightarrow \neg \neg \psi
$$

we can hence freely use the law of excluded middle in constructive arguments - but at the price that the conclusion $\neg \neg \psi$ is doubly negated, which is why typically this observation is not of much use.

However, double negation stability of equality does allow us to conclude $\psi$ from the a priori much weaker premiss $\neg \neg \psi$, if $\psi$ is of the form $\left(s={ }_{A \sim t}\right)$.

This stability is a consequence of the field property. In case that the ring $A$ is not reduced and hence $A^{\sim}$ is not a field, a substitute is given in Exercise 35. We stress that the proof of statement $(\mathbb{F})$ does not require double negation stability of $A$ on the metalevel; in fact, it is fully constructive.

Proposition 31. If $A$ is reduced, then the sheaf of rings $A^{\sim}$ has $\neg-$-stable equality:

$$
\operatorname{Spec}(A) \models \forall x, y: A^{\sim} . \neg \neg(x=y) \Rightarrow x=y .
$$

Proof. We argue internally in the universe of sheaves, using the sheaf semantics. Let $x, y: A^{\sim}$ be given such that $\neg \neg(x=y)$. We will verify that $x-y$ is not invertible; by the field condition, this will imply $x-y=0$, hence $x=y$.

So assume that $x-y$ is invertible. Then $\neg(x-y=0)$, since if $x-y=0$, then $1=(x-y)(x-y)^{-1}=0$ in contradiction to $1 \neq 0$. Hence we have a contradiction to the assumption $\neg \neg(x-y=0)$. 
The Noetherian property. Noetherian conditions are notoriously tricky in constructive algebra, not least because of their overuse in classical commutative algebra which render attempts to extract constructive content more challenging.

There are several proposals for constructively sensible definition of Noetherian rings in the literature on constructive algebra, each with unique advantages and disadvantages [26, 81, 85. Insightful comments on why this is so can be found in the introduction and more specifically on page 27 of the textbook by Henri Lombardi and Claude Quitté [14. To this list of conditions, we add the following.

Definition 32. A ring is anonymously Noetherian if and only if any of its ideals is not not finitely generated.

Example 33. The assumption that any ideal of $\mathbb{Z}$ is finitely generated implies the law of excluded middle. But we can prove, constructively, that $\mathbb{Z}$ is anonymously Noetherian: Let $\mathfrak{a}$ be an ideal of $\mathbb{Z}$ and assume that $\mathfrak{a}$ is not finitely generated. Then $1 \notin \mathfrak{a}$, as else $\mathfrak{a}=(1)$ would be finitely generated. Also $2 \notin \mathfrak{a}$, as else (noting that $1 \notin \mathfrak{a}$ ), $\mathfrak{a}=(2)$. Continuing in this manner, we may conclude $n \notin \mathfrak{a}$ for every number $n \geq 1$. Hence $\mathfrak{a}=(0)$. But this is a contradiction to the assumption that $\mathfrak{a}$ is not finitely generated.

Definition 32 is at odds with the idea that constructive mathematics should be informative: It only expresses that it is impossible for no finite generating family to exist without requiring that such a family should be given - it may remain anonymous 0 And while Hilbert's basis theorem, stating that if a ring $B$ is Noetherian then so is the polynomial $\operatorname{ring} B[X]$, does have a constructive proof for the anonymous version of the Noetherian condition $P$ this result seems empty: For it just transforms one unconcrete promise (generators of each ideal of $B$ exist somewhere, platonically) into another (generators for ideals of $B[X]$ exist somewhere, platonically).

Curiously, the anonymous version of the Noetherian condition is still useful in constructive algebra when interpreted by the sheaf semantics: This is firstly because it just so happens that $A^{\sim}$ is anonymously Noetherian

\footnotetext{
"We borrowed the term "anonymous" from type theory, where it is used with a similar meaning (see for instance Ref. [86]). However, there is a subtle difference: The conjunction of "there is at most one element $x$ " and "there is anonymously an element $x$ in the sense of type theory" implies that there actually exists an element $x$. In contrast, not not existence does not.

pFor instance, a careful reading of the textbook proof given in [87, Theorem 7.5] shows that the basis theorem holds intuitionistically as stated.
} 
if $A$ is reduced, and secondly because the interpretation of double negation with the sheaf semantics does have nontrivial content (as evidenced by statement $(\mathrm{F})$ ). The Noetherian condition is also a unique feature of the sheaf model, not shared by the ring $A$ or its stalks.

Proposition 34. If $A$ is reduced, then the sheaf of rings $A^{\sim}$ is anonymously Noetherian.

Proof. We argue internally, under the sheaf semantics. Let $\mathfrak{a} \subseteq A^{\sim}$ be an ideal. Assume that $\mathfrak{a}$ is not finitely generated. We will verify that then $\mathfrak{a}=(0)$, hence that $\mathfrak{a}$ is finitely generated; contradiction.

Let $x \in \mathfrak{a}$. If $x$ is invertible, then $\mathfrak{a}=(1)$ is finitely generated. Hence $x$ is not invertible. Thus $x=0$ because $A^{\sim}$ is a field.

Remark 35. The condition for a ring to be (anonymously) Noetherian is a higher-order condition and hence out of scope of the first-order version of the sheaf semantics presented in Section 2.4. However, the general version of the sheaf semantics is sound with respect to intuitionistic higher-order logic, hence the proof of Proposition 34 is valid.

\subsection{An algebraic origin story}

The purpose of this section is to properly motivate the construction leading to $A^{\sim}$. Briefly, the story is as follows, with details given below.

(1) Given a ring $A$, we set out to construct the free local ring over $A$.

(2) In the strict sense of the word, this endeavor will fail, but in a wider sense the quest will succeed and yield the sheaf $A^{\sim}$.

(3) It is then a surprising observation that $A^{\sim}$ is a field, even though we only set out to construct a local ring.

(4) The field property of $A^{\sim}$ should be appropriately appreciated: While there is a general machinery for free constructions of this generalized kind, this machinery is not applicable for constructing free fields.

Free constructions. Let $G$ be a group. Then there is a universal way of turning $G$ into an abelian group $G^{\text {ab }}$ equipped with a group homomorphism $G \rightarrow G^{\mathrm{ab}}$, the free abelian group over $G$. This abelianization has the universal property that for any homomorphism $G \rightarrow M$ into an abelian 
group, there is exactly one homomorphism $G^{\mathrm{ab}} \rightarrow M$ rendering the diagram

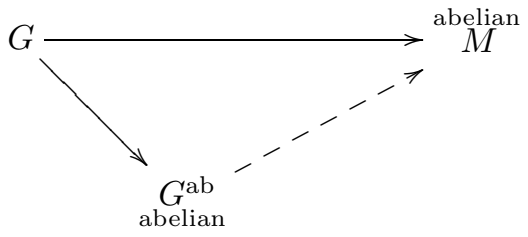

commutative. In textbooks, the abelianization is usually constructed as $G^{\mathrm{ab}}=G /[G, G]$, that is as the quotient by the subgroup generated by the commutators. This explicit construction makes it appear that abelianization is a specific result in algebra pertaining to groups.

We can also cast the construction in logical language. This change in perspective makes it evident that abelianization is just a special case of a more general procedure. The free abelian group over $G$ can be obtained as the term model of the theory of abelian groups extended by a constant $x_{g}$ for each element $g \in G$ and the axioms

$$
\begin{array}{lr}
\top \vdash x_{g \circ h}=x_{g} \circ x_{h} & \text { (for each } g, h \in G \text { ) } \\
\top \vdash x_{g^{-1}}=x_{g}^{-1} & \text { (for each } g \in G \text { ) } \\
\top \vdash x_{e}=e &
\end{array}
$$

That is, $G^{\text {ab }}$ contains exactly those elements and exactly those identifications between elements such that $G^{\mathrm{ab}}$ is an abelian group and such that $G$ can be interpreted in it (that is, that there is a homomorphism $G \rightarrow G^{\mathrm{ab}}$ ), and nothing more.

In exactly the same manner, we can construct the free group over a monoid, the free $\mathbb{Z} /(2)$-algebra over a ring, the free ring over a set or the free Heyting algebra over a partially ordered set. This is all well-understood; a particularly perspicuous account in the general setting of partial Horn theories is due to Erik Palmgren and Steve Vickers [88, Section 5].

However, this procedure cannot be carried out for any pair of theories. For instance, there are no free fields. The free field on a set $M$ would be a field $K$ together with a map $M \rightarrow K$ such that any map $M \rightarrow$ $L$ into a field $L$ factors over the given map $M \rightarrow K$ by a unique field homomorphism $K \rightarrow L$.

For instance, in the case $M=\{t\}$, the field $\mathbb{Q}(t)$ of rational functions comes close, but fails because there are no field homomorphisms $\mathbb{Q}(t) \rightarrow L$ which could map $t$ to an algebraic element. We could also try to concoct a term model, but the set of terms in the language of a field extended by a constant $t$ modulo provable equivalence is only the ring $\mathbb{Z}[t]$, not a field. 
The non-existence of free fields is put into perspective by the observation that the theory of fields is, for any of its variants, not a partial Horn theory.

Free local rings. The theory of local rings, that is the theory of rings extended by the two coherent axioms

$$
\begin{gathered}
1=0 \vdash \perp \\
(\exists z: R .(x+y) z=1) \vdash_{x: R, y: R}(\exists z: R . x z=1) \vee(\exists z: R . y z=1),
\end{gathered}
$$

is also not a partial Horn theory. Hence there is no reason to expect that free local rings exist, and indeed, in general they do not. However, despite this negative outlook, we want to analyze the situation in more detail.

The correct notion of a morphism between local rings is that of a local ring homomorphism, a ring homomorphism $f: R \rightarrow S$ which reflects invertibility, that is for which $f(x)$ being invertible implies that $x$ is invertible.

Hence a free local ring over a ring $A$ is to be a local ring $A^{\prime}$ together with a ring homomorphism $A \rightarrow A^{\prime}$ such that the following universal property holds: Any ring homomorphism $A \rightarrow B$ into a local ring uniquely factors via a local ring homomorphism over $A \rightarrow A^{\prime}$ as indicated in the diagram.

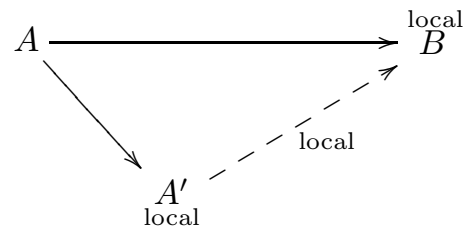

To develop intuition for what this universal property requests, let a homomorphism $f: A \rightarrow B$ into a local ring be given. The subset $B^{\times} \subseteq B$ is a prime filter, due to the locality of $B$. Its preimage $\mathfrak{f}:=f^{-1}\left[B^{\times}\right]$is a prime filter of $A$. Hence the localization $A_{\mathfrak{f}}=A\left[\mathfrak{f}^{-1}\right]$ is a local ring and $f$ factors via the well-defined map $f^{\prime}: A_{\mathfrak{f}} \rightarrow B, \frac{x}{s} \mapsto f(s)^{-1} f(x)$ over the localization morphism $A \rightarrow A_{\mathfrak{f}}, x \mapsto \frac{x}{1}$. Furthermore, this map is local.

Hence the stalks $A_{\mathfrak{f}}$, where $\mathfrak{f}$ ranges over the prime filters of $A$, can be regarded as approximations to the hypothetical true local ring over $A$. Each of these stalks is local, but might not validate the universal property for all homomorphisms $A \rightarrow B$ into local rings. We are thus led to the following conclusions.

(1) A free local ring $A \rightarrow A^{\prime}$ over $A$ exists if $A$ has exactly one prime filter. (The converse also holds.) In classical mathematics, this condition is 
satisfied if and only if $A$ is local ring of Krull dimension zero. In this case, the unique prime filter is $A^{\times}$and the free local ring over $A$ is given by $A$ itself. Hence in general, the problem of constructing the free local ring over a given ring is not solvable.

(2) However, we could solve the problem in full generality if we would have a special prime filter $\mathfrak{f}_{0}$ which could somehow shift shape, that is, turn into any specific prime filter $\mathfrak{f}$ on demand.

The existence of such a shape-shifting prime filter $\mathfrak{f}_{0}$ is not a pipe dream. While it does not exist as a set, it can be realized as a sheaf.

Definition 36. The generic prime filter $\mathfrak{f}_{0}$ of a ring $A$ is the generic model of the theory of prime filters of $A$.

According to this definition, the generic prime filter is a particular sheaf model over the classifying locale of prime filters of $A$, the spectrum $\operatorname{Spec}(A)$ of $A$. By localizing at the generic prime filter, we solve the problem of constructing the free local ring in a generalized sense: The free local ring over $A$ exists as a certain sheaf of rings. It has a universal property not only with respect to all ordinary rings, but also with respect to all sheaves of rings on arbitrary locales.

Constructing the sheaf $\boldsymbol{A}^{\sim}$. We recall from Section 2.3 that the given ring $A$ has a simple counterpart in the universe of sheaves over $\operatorname{Spec}(A)$, namely the constant sheaf $\underline{A}$. We construct the sheaf $A^{\sim}$ as

$$
A^{\sim}:=\underline{A}\left[\mathfrak{f}_{0}^{-1}\right],
$$

that is as the localization of $\underline{A}$ at its prime filter $\mathfrak{f}_{0}$.

With this definition, a number of statements from Section 3.2 are immediate.

Proposition 37. Let $A$ be a ring. Then the statements (B), (C) and (D) displayed above hold:

\footnotetext{
qThis description contains a slight abuse of notation. According to Definition 36 the generic prime filter $\mathfrak{f}_{0}$ is a model of the theory of prime filters, hence a family $\left(U_{x}\right)_{x \in A}$ of opens of $\operatorname{Spec}(A)$, one for each nullary relation symbol of the theory. In fact, by the construction of the generic model, the open $U_{x}$ is simply the element $[D(x)]$ of the Lindenbaum algebra. We turn this family into the subsheaf of $\underline{A}$ given by $U \mapsto\{g \in$ $\left.\operatorname{Hom}(U, A) \mid \forall x \in A \cdot g^{-1}[\{a\}] \preceq[D(a)]\right\}$. Abusing notation, we denote this subsheaf also as "fo" $\mathfrak{f}_{0}$ ". The statement " $\mathfrak{f}_{0}$ is a prime filter" is then validated by the sheaf semantics.
} 
(B) The ring $A^{\sim}$ has every property $\varphi$ that $A$ has, assuming that $\varphi$ is a property of rings which can be put in the formal higher-order language of toposes; that there is an intuitionistic proof that $\varphi$ is localizationstable; and that every element of $A$ is nilpotent or not.

(C) If a geometric sequent holds for $A^{\sim}$, then it also holds for all stalks $A_{\mathfrak{f}}$ at prime filters. The converse holds if $\operatorname{Spec}(A)$ is spatial, for instance if BPIT is available.

(D) The ring $A^{\sim}$ is local.

Proof. (D) The ring $A^{\sim}$ is local as it is the localization at a prime filter.

(B) The assumption on $A$ implies that $\operatorname{Spec}(A)$ is overt. For overt locales, it is a standard fact that any (infinitary) first-order property of sets passes to the induced constant sheaves. Hence the claim follows by observing that $A^{\sim}$ is a localization of $\underline{A}$.

(C) We recall that the points of $\operatorname{Spec}(A)$ are precisely the prime filters of $A$. The sheaf-theoretic stalk of the constant sheaf $\underline{A}$ at any point is precisely $A$, and by construction, the stalk of the generic prime filter $\mathfrak{f}_{0}$ (considered as a subsheaf of $\underline{A}$ ) at a prime filter $\mathfrak{f}$ is $\mathfrak{f}$. Since localization is a geometric construction, it is preserved by the operation of computing (sheaf-theoretic) stalks. Hence the stalk of $A^{\sim}$ at a point $\mathfrak{f}$ is $A\left[\mathfrak{f}^{-1}\right]$, that is, the ring-theoretic stalk of $A$ at $\mathfrak{f}$. The claim therefore follows from Proposition 28 .

Furthermore, the sheaf $A^{\sim}$ is the free local ring over $A$ in the following sense. If $R$ is a sheaf of rings on a locale $X$ and $S$ is a sheaf of rings on a locale $Y$, then by a morphism $R \rightarrow S$ we mean a pair consisting of a morphism $f: Y \rightarrow X$ of locales together with a morphism $f^{\sharp}: f^{-1} R \rightarrow S$ of sheaves of rings on $Y$. (The sheaf $f^{-1} R$ is the pullback sheaf of $R$ along $f$.) In this way a category of sheaves of rings on arbitrary locales is set up, into which the category of ordinary rings embeds.

For $A^{\sim}$ to be the local ring over $A$, there has to be a morphism $A \rightarrow$ $A^{\sim}$. For this we take the pair $\left\langle g, g^{\sharp}\right\rangle$ where $g: \operatorname{Spec}(A) \rightarrow$ pt is the unique morphism into the one-point locale and $g^{\sharp}: g^{-1} A=\underline{A} \rightarrow A^{\sim}$ is the localization morphism.

If $\left\langle f, f^{\sharp}\right\rangle: A \rightarrow B$ is a morphism into a local sheaf of rings on an arbitrary locale $X$, we can construct, in the universe of sheaves over $X$, the factorization $\underline{A} \rightarrow \underline{A}\left[\mathfrak{f}^{-1}\right] \rightarrow B$ where $\mathfrak{f}:=\left(f^{\sharp}\right)^{-1}\left[B^{\times}\right]$. Since $\mathfrak{f}$ is a model of the theory of prime filters of $A$, there is a unique morphism $g: X \rightarrow \operatorname{Spec}(A)$ of locales such that $g^{-1} \mathfrak{f}_{0}=\mathfrak{f}$. Since $g^{-1} A^{\sim}=\underline{A}\left[\mathfrak{f}^{-1}\right]$, the localization just 
constructed gives rise to a morphism $A^{\sim} \rightarrow B$ of local sheaves of rings.

Remark 38. There is general topos-theoretic machinery for generalized free constructions of this kind [89, 90, and this machinery can be used to construct several kinds of spectra, including the ordinary spectrum of a ring. However, this machinery does have limitations, and in particular it cannot be used to construct free fields.

Remark 39. Given a ring $A$, there is the geometric theory of local localizations of $A$. This is the theory of local rings extended by constants $e_{a}$ for each element $a \in A$ and the following axioms:

$$
\begin{aligned}
& \top \vdash e_{0}=0 \\
& \top \vdash e_{1}=1 \\
& \top \vdash e_{a+b}=e_{a}+e_{b} \quad \text { (for each } a, b \in A \text { ) } \\
& \top \vdash e_{a b}=e_{a} e_{b} \quad(\text { for each } a, b \in A) \\
& \top \vdash e_{-a}=-e_{a} \quad \text { (for each } a \in A \text { ) } \\
& \top \vdash \vdash_{x: R} \bigvee_{a \in A} \bigvee_{s \in A}\left(\left(\exists z: R . e_{s} z=1\right) \wedge e_{s} x=e_{a}\right) \\
& \left.e_{a}=0 \vdash \bigvee_{\substack{s \in A \\
s a=0}}\left(\exists z: R \cdot e_{s} z=1\right) \quad \text { (for each } a \in A\right)
\end{aligned}
$$

By the first five listed entries, a model $R$ of this theory comes equipped with a ring homomorphism $f: A \rightarrow R$. The remaining two entries ensure that $R$ is a localization of $A$, more precisely that it is the localization of $A$ at $f^{-1} R^{\times}$.

Since this theory is not propositional, Section 2.2 does not apply. There is, however, still a classifying topos of this theory, and it turns out that it coincides with the topos of sheaves over $\operatorname{Spec}(A)$ - mainly because the theory of local localizations of $A$ is equivalent to the theory of prime filters of $A$. Under this identification, the generic model of the local localization is precisely the sheaf $A^{\sim}$, and it validates first-order sequents not expected from a local localization; as repeatedly stressed (statement $(\mathrm{E})$ ), it is a field, even though the theory of local localizations does not prove the field condition.

\subsection{Understanding the sheaf model}

In Section 3.3 we constructed the sheaf $A^{\sim}$ as the free local ring over $A$, more specifically as the localization of the constant sheaf $\underline{A}$ on $\operatorname{Spec}(A)$ 
at the generic prime filter $\mathfrak{f}_{0}$ of $A$. Several of the properties mentioned in Section 3.2 are immediate consequences of this abstract description.

However, for a more detailed identification of $A^{\sim}$ - especially for verifying its field property - we require a more concrete description. In particular, we require a concrete description of the underlying frame of $\operatorname{Spec}(A)$. Since this frame is defined as the Lindenbaum algebra of the theory of prime filters of $A$, we need a thorough grasp of the set of sequents proved by this theory.

Such an understanding is imparted by the following theorem. It is the central workhorse of our approach.

Theorem 40. Let $A$ be a ring. The Lindenbaum algebra of the theory $\mathbb{T}$ of prime filters of $A$ is canonically isomorphic to the frame of radical ideals in $A$, via an isomorphism which maps

$$
[D(f)] \longmapsto \sqrt{(f)} .
$$

In particular:

$$
\mathbb{T} \text { proves } D(f) \vdash \bigvee_{i \in I} D\left(g_{i}\right) \quad \text { iff } \quad f \in \sqrt{\left(g_{i}\right)_{i}} .
$$

Proof. The set of radical ideals $\operatorname{Rad}(A)$ is ordered by inclusion. Its least element is $\sqrt{(0)}$, the meet $\mathfrak{a} \wedge \mathfrak{b}$ of two radical ideals is their intersection and the join $\bigvee_{i} \mathfrak{a}_{i}$ of radical ideals is $\sqrt{\sum_{i} \mathfrak{a}_{i}}$. In this way the radical ideals form a frame.

The Lindenbaum algebra of $\mathbb{T}$ is the free frame generated by the symbols $D(x)$, where $x$ ranges over the elements of $A$, modulo the axioms for a prime filter. Hence to give a frame homomorphism $\mathcal{O}(\operatorname{Spec}(A)) \rightarrow \operatorname{Rad}(A)$, we need to specify images in $\operatorname{Rad}(A)$ for each generator in such a way that the axioms for a prime filter are satisfied. This is achieved by declaring $D(f) \mapsto \sqrt{(f)}$ :

$$
\begin{aligned}
& D(0) \vdash \perp \\
& \sqrt{(0)} \subseteq \sqrt{(0)} \\
& D(f+g) \vdash D(f) \vee D(g) \\
& \sqrt{(f+g)} \subseteq \sqrt{\sqrt{(f)}+\sqrt{(g)}} \\
& \top \vdash D(1) \\
& \sqrt{(1)} \subseteq \sqrt{(1)} \\
& D(f) \wedge D(g) \dashv \vdash D(f g) \\
& \sqrt{(f)} \cap \sqrt{(g)}=\sqrt{(f g)}
\end{aligned}
$$

The most interesting of these radical inclusions is probably $\sqrt{(f)} \cap \sqrt{(g)} \subseteq$ $\sqrt{(f g)}$, as this inclusion illustrates why passing to the radical is vital; in general, we do not have $(f) \cap(g) \subseteq(f g)$. 
The frame homomorphism obtained in this manner is surjective, since a preimage for a radical ideal $\mathfrak{a}$ is given by $\bigvee_{f \in \mathfrak{a}}[D(f)]$. By Exercise [5, it remains to show that the homomorphism reflects the ordering.

To this end, let an element $f \in A$ and a family $\left(g_{i}\right)_{i \in I}$ of elements be given and assume $\sqrt{(f)} \subseteq \sqrt{\sum_{i} \sqrt{\left(g_{i}\right)}}$. Then there are values $n, m \in \mathbb{N}$, $i_{1}, \ldots, i_{m} \in I$ and $u_{1}, \ldots, u_{m} \in A$ such that $f^{n}=u_{1} g_{i_{1}}+\cdots+u_{m} g_{i_{m}}$. We then have the following chain of entailments.

$$
D(f) \vdash D\left(f^{n}\right) \vdash \bigvee_{k=1}^{m} D\left(u_{k} g_{i_{k}}\right) \vdash \bigvee_{k=1}^{m} D\left(g_{i_{k}}\right) \vdash \bigvee_{i} D\left(g_{i}\right)
$$

Theorem 40 shows that any derivation in the theory of prime filters of $A$ can be put into a normal form. An expression of the form " $f^{n}=$ $u_{1} g_{i_{1}}+\cdots+u_{m} g_{i_{m}}$ " can be regarded as an algebraic certificate of the entailment $D(f) \vdash \bigvee_{i} D\left(g_{i}\right)$.

Remark 41. An immediate corollary of Theorem 40 is that $\operatorname{Spec}(A)$ is compact, since if $f \in \sqrt{\left(g_{i}\right)_{i}}$ then also $f \in \sqrt{\left(g_{i_{1}}, \ldots, g_{i_{m}}\right)}$ for suitable indices $i_{1}, \ldots, i_{m}$. However, the stronger result that $\operatorname{Spec}(A)$ is even a coherent locale can also be deduced from a general theorem since the theory of prime filters is a coherent theory (Exercise 24).

Remark 42. A further corollary of Theorem 40 is that the theory of prime filters of $A$ is inconsistent (that it, that it proves the sequent $\top \vdash \perp$, which can also be written as $D(1) \vdash D(0)$ ) if and only if $A$ is the trivial ring. Contrapositively, if $A$ is not the trivial ring, then the theory is consistent. This observation can be regarded as a finitary substitute of the statement (equivalent to BPIT in general) "nontrivial rings contain prime filters".

Remark 43. Given the importance of Theorem 40, it is natural to wonder where the proof stems from. Our proof proceeded in an ad hoc fashion, verifying that the frame of radical ideals has the required properties - but where does the idea to use radical ideals come from? In general, determining the set of provable sequents of a geometric theory is a challenging problem. Our most efficient tool is probably the theory of entailment relations. We refer to Refs. [30, 31] and the references therein and also strongly recommend the literature on dynamical methods in commutative algebra [28, 29, 32.

Having identified the underlying frame of $\operatorname{Spec}(A)$, the second workhorse is an explicit description of the sheaf $A^{\sim}$.

Proposition 44. Let $A$ be a ring. For any $f \in A$, there is a canonical 
isomorphism

$$
A\left[f^{-1}\right] \longrightarrow A^{\sim}([D(f)]) .
$$

In particular, for $f=1$, we obtain statement (A) from Section 3.2.

Proof. For $f \in A$, we set $S_{f}:=\left\{g \in A \mid \exists n \in \mathbb{N} . \exists u \in A\right.$. $\left.f^{n}=u g\right\} \subseteq A$. This multiplicative system is the saturation of $\left\{f^{0}, f^{1}, \ldots\right\}$. The localization $A\left[S_{f}^{-1}\right]$ is canonically isomorphic to $A\left[f^{-1}\right]$, but has the advantage that for elements $f, g \in A$ such that $\sqrt{(f)}=\sqrt{(g)}$, the rings $A\left[S_{f}^{-1}\right]$ and $A\left[S_{g}^{-1}\right]$ are actually the same while $A\left[f^{-1}\right]$ and $A\left[g^{-1}\right]$ are merely canonically isomorphic. This property eases formalization in set theory; it is otherwise not important.

On the basis of $\operatorname{Spec}(A)$ given by the opens of the form $[D(f)]$, we define a partial presheaf by

$$
[D(f)] \longmapsto A\left[S_{f}^{-1}\right] .
$$

This partial presheaf is, on the basis where it is defined, a sheaf. Unraveling the definitions, this claim boils down to the following basic result in commutative algebra:

Let $B$ be a ring. Let $1=f_{1}+\cdots+f_{m} \in B$ be a partition of unity. Let elements $s_{i} \in B\left[f_{i}^{-1}\right]$ be given. Assume that $s_{j}=s_{k}$ in $B\left[\left(f_{j} f_{k}\right)^{-1}\right]$ for all pairs of indices. Then there is exactly one element $s \in B$ such that, for all indices $i, s=s_{i}$ in $B\left[f_{i}^{-1}\right]$.

The remainder of the argument is by sheaf-theoretic generalities.

More generally, for any $A$-module $M$, we can construct the localization $M^{\sim}:=\underline{M}\left[\mathfrak{f}_{0}^{-1}\right]$. In the special case that $M$ is $A$, this definition coincides with our original definition of $A^{\sim}$. The proof of Proposition 44 carries over to show that $M^{\sim}([D(f)]) \cong M\left[f^{-1}\right]$.

An elementary reformulation. As a corollary of Theorem 40 and Proposition 44, we can unwind the definitions to recast the sheaf semantics in entirely explicit algebraic terms, with no locales or sheaves in sight. Notwithstanding the impredicative nature of locale theory, the resulting formulation will even make sense in metatheories without a powerset operation such as CZF [91, 92] or arithmetic universes [34, 35]. In fact, it is then a purely syntactic translation procedure which can be carried out within PRA.

The approach using locales and sheaves provides two ingredients which go beyond mere syntax: Firstly, they give motivation for setting up the 
semantics in the way we do (Section 3.3). Secondly, they conceptualize $A^{\sim}$ as a single entity, just as rings like $A / \mathfrak{m}$ or $A_{\mathfrak{p}}$ are single entities; nevertheless, after unrolling the definitions, any statement about $A^{\sim}$ is merely a (logically more complex) statement about $A$ and its localizations $A\left[f^{-1}\right]$.

By a formula over a ring element $f \in A$, we mean a first-order formula over the signature which has

(1) a sort " $A \sim$ " and function symbols for the structure of a ring,

(2) for each $A$-module $M$, a sort " $M \sim$ " and function symbols for the structure of an $A^{\sim}$-module,

(3) for each linear map $M \rightarrow N$, a function symbol $M^{\sim} \rightarrow N^{\sim}$,

(4) a constant of sort $A^{\sim}$ for each element of $A\left[f^{-1}\right]$ and

(5) for any $A$-module $M$, a constant of sort $M^{\sim}$ for each element of $M\left[f^{-1}\right]$.

Corollary 45. Let $A$ be a ring. Let $\varphi$ be a formula over $f \in A$. Then $[D(f)] \models \varphi$ in the sense of Table 5 if and only if $f=\varphi$ in the sense of Table 6 .

Proof. Induction on the structure of $\varphi$, harnessing the explicit descriptions provided by Theorem 40 and Proposition 44.

Table 6. A purely algebraic presentation of the sheaf semantics of $\operatorname{Spec}(A)$

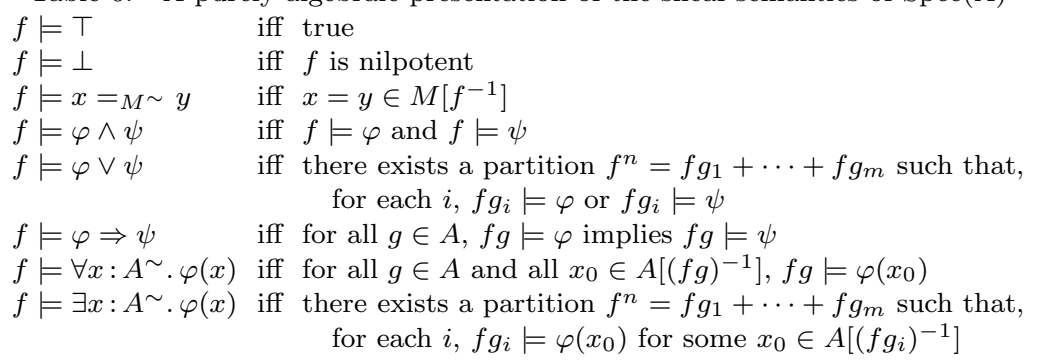

The analogue of Theorem 22 for the new formulation of the sheaf semantics reads as follows.

Theorem 46. Let $A$ be a ring. Let $f \in A$. Let $\varphi$ be a formula over $f$.

(1) Let $g \in A$. If $f=\varphi$, then $f g \models \varphi$.

(2) Let $f^{n}=f g_{1}+\cdots+f g_{m}$. If $f g_{i}=\varphi$ for all indices $i$, then $f=\varphi$.

(3) Let $\psi$ be a further formula over $f$. If $f \models \varphi$ and if $\varphi$ entails $\psi$ intuitionistically, then also $f \models \psi$. 
Proof. Combination of Theorem 22 and Corollary 45, or alternatively direct proof by induction without using any preliminaries from earlier sections of this contribution.

The field property. We are now in a position to verify statement (E) of Section 3.2

Proposition 47. Let $A$ be a reduced ring. Then $A^{\sim}$ is a field in that

$$
1 \models \forall x: A^{\sim} .\left(\left(\neg\left(\exists y: A^{\sim} . x y=1\right)\right) \Rightarrow x=0\right) .
$$

Proof. By Table 6, we have to verify the following claim.

For any element $f \in A$ and any element $x \in A\left[f^{-1}\right]$,

for any element $g \in A$,

if for any element $h \in A$,

if there is $(f g h)^{n}=\sum_{i=1}^{m} f g h p_{i}$ such that for each $i$, there exists $y \in A\left[\left(f g h p_{i}\right)^{-1}\right]$ such that

then $f g h$ is nilpotent,

$$
x y=1 \text { in } A\left[\left(f g h p_{i}\right)^{-1}\right] \text {, }
$$

$$
\text { then } x=0 \text { in } A\left[(f g)^{-1}\right] \text {. }
$$

Hence let elements $f \in A, x \in A\left[f^{-1}\right]$ and $g \in A$ be given and assume that for any $h \in A$, the displayed condition holds. We are to show that $x=0$ in $A\left[(f g)^{-1}\right]$.

Write $x=\frac{x^{\prime}}{f}$. For $h:=x^{\prime}$, we have the partition $(f g h)^{1}=f g h \cdot 1$ with $m=1$ and $p_{1}=1$. For $y:=\frac{f}{x^{\prime}}=\frac{f^{2} g p_{1}}{f g h p_{1}}$, we have $x y=1$ in $A\left[\left(f g h p_{1}\right)^{-1}\right]$. By assumption, the element $f g h$ is nilpotent, hence zero. Thus $x^{\prime}=0$ in $A\left[(f g)^{-1}\right]$. This implies that $x=0$ in $A\left[(f g)^{-1}\right]$.

The proof of Proposition 47 visibly demonstrates that unwinding the definition of the sheaf semantics quickly results in formulas which are quite convoluted. This behavior is a basic consequence of the fact that, with the exception of the clauses for " $\top$ " and " $\wedge$ ", all clauses in Table [ introduce additional quantifiers.

This increase in complexity should be properly appreciated: The sheaf semantics allows us to harness convoluted properties in simple language. The unrolled monstrosity in the proof of Proposition 47 is not memorable at all and unlikely to find its way into human-written proofs. However, in the form " $A^{\sim}$ is a field", it becomes easily accessible. This state of affairs becomes the more pronounced the more negations and especially double 
negations occur in the proof.

Example 48. For future reference, and also for giving a different perspective on statement (F), we unroll here the interpretation of $f \models \neg \neg \varphi$. It is:

For any element $g \in A$,

if for any element $h \in A$, if $f g h \models \varphi$,

then $f g h$ is nilpotent,

then $f g$ is nilpotent.

In the case that $A$ is reduced and $f$ is the unit element of $A$, this condition implies (by setting $g:=1$ ): "If $h=0$ is the only element of $A$ such that $h \models$ $\varphi$, then $1=0$ in $A$."

\subsection{Evaluating test cases}

Size of injective and surjective matrices. On page 4 , we presented a classical proof of the following theorem.

Theorem. Let $M$ be an injective matrix with more columns than rows over a reduced $\operatorname{ring} A$. Then $1=0$ in $A$.

We are now in a position to show how the reduction technique developed in Section 3 can be used to recast the classical proof so that it becomes constructive:

Proof. Since localization is exact, the matrix $M$ is also injective when considered as a matrix over $A^{\sim}$ (statement $(\underline{B})$ ). Since $A^{\sim}$ is a field, this is a contradiction to basic (intuitionistic) linear algebra $\mathrm{f}$ In other words, $1 \models \perp$. By Remark 42 or Table 6 this amounts to $1=0$ in $A$.

If desired, the proof can be mechanically unwound to a fully explicit proof which does not refer to the sheaf semantics. In this case, unwinding yields exactly the proof by Fred Richman [13, Theorem 2] (only that he needs one additional paragraph to deal with the possibility that the ring is not reduced, which we for conciseness simply assumed):

\footnotetext{
${ }^{\mathrm{r}}$ We are referencing the following theorem: "Let $M$ be an injective matrix with more columns than rows over a field $k$ in the sense that any element of $k$ is either zero or invertible. Then $\perp$." This theorem is proven by repeatedly applying elementary row and column transformations until the matrix is of the form $\left(\begin{array}{ll}I & 0 \\ 0 & 0\end{array}\right)$. By assumption on the size of $M$, there is at least one zero column. This contradicts injectivity.

However, since the ring $A \sim$ is only a field in the weaker sense that nonunits are zero, this theorem does not apply directly. We only have that any element of $A^{\sim}$ is not not zero or invertible. We hence employ the trick described in the section on double negation stability (page 49).
} 
Proof. Over the localized ring $A\left[M_{i j}^{-1}\right]$, the matrix element $M_{i j}$ is invertible and hence $M$ can be put into the form $\left(\begin{array}{cc}1 & 0 \\ 0 & M^{\prime}\end{array}\right)$ by elementary row and column transformations. The matrix $M^{\prime}$ is injective and has more columns than rows, hence by induction, applied to this submatrix over $A\left[M_{i j}^{-1}\right]$, we have $1=0$ in $A\left[M_{i j}^{-1}\right]$. Since $A$ is reduced, this amounts to $M_{i j}=0$ in $A$.

Hence $M$ is the zero matrix. Thus $M(1,0, \ldots, 0)^{t}=0$ and by injectivity $1=0$ in $A$.

In exactly the same fashion, the dual statement about surjective matrices can be proven.

Concluding, one can well ask what the computational content of the equation $1=0$ is. The answer depends on the ring in question. In rings where we know a priori that $1 \neq 0$, the two statements are most useful in their contrapositive form. But in rings of the form $R\left[f^{-1}\right]$ or $R /(f)$, learning that $1=0$ can indeed be useful: In the former case, this tells us that $f$ is nilpotent (and a computational witness of $1=0$ can be transformed into a number $n$ such that $f^{n}=0$ ), and in the latter case, this tells us that $f$ is invertible.

Grothendieck's generic freeness lemma. This theorem is a basic theorem in algebraic geometry, used for instance for developing the theory of moduli spaces. When phrased for the affine situation in a constructively sensible way, a simple version of this theorem reads as follows. We recall from Section 3.1 that the notation $M\left[f^{-1}\right]$ makes sense for any element $f \in A$; in case $f$ is zero or nilpotent, the localized module $M\left[f^{-1}\right]$ will be the trivial module.

Theorem. Let $A$ be a reduced ring. Let $M$ be a finitely generated $A$ module. If $f=0$ is the only element of $A$ such that $M\left[f^{-1}\right]$ is a free $A\left[f^{-1}\right]$-module, then $1=0$ in $A$.

This theorem is particularly interesting for the program of constructivizing classical commutative algebra, as it is an example where the constructive proof is much shorter and arguably more perspiciuous than classical proofs (for which see for instance Refs. [93, Lemme 6.9.2], [94, Thm. 24.1] or [95, Thm. 14.4], which only cover the case of Noetherian integral domains, or Refs. [96, 97, Tag 051Q] which proceed in a series of intermediate steps, reducing to that case).

Proof. By Example 48 and Exercise 36(b), the claim amounts to the statement that $M^{\sim}$ is not not free. Since $A^{\sim}$ is a field, this statement 
follows from basic (intuitionistic) linear algebras

Again, the proof can be unwound if desired [98, Proposition 3]:

Proof. We proceed by induction on the length of a given generating family of $M$. Let $M$ be generated by $\left(v_{1}, \ldots, v_{m}\right)$.

We show that the family $\left(v_{1}, \ldots, v_{m}\right)$ is linearly independent. Let $\sum_{i} a_{i} v_{i}=0$. Over $A\left[a_{i}^{-1}\right]$, the vector $v_{i} \in M\left[a_{i}^{-1}\right]$ is a linear combination of the other generators. Thus $M\left[a_{i}^{-1}\right]$ can be generated as an $A\left[a_{i}^{-1}\right]$-module by fewer than $m$ generators. The induction hypothesis, applied to this module over $A\left[a_{i}^{-1}\right]$, yields that $1=0$ in $A\left[a_{i}^{-1}\right]$. Since $A$ is reduced, this amounts to $a_{i}=0$.

We finish by using the assumption for $f=1$.

The full version of Grothendieck's generic freeness lemma, and a constructive proof of it, read as follows.

Theorem. Let $A$ be a reduced ring. Let $B$ be an $A$-algebra of finite type. Let $M$ be a finitely generated $B$-module. If $f=0$ is the only element of $A$ such that

(1) $B\left[f^{-1}\right]$ and $M\left[f^{-1}\right]$ are free modules over $A\left[f^{-1}\right]$,

(2) $A\left[f^{-1}\right] \rightarrow B\left[f^{-1}\right]$ is of finite presentation and

(3) $M\left[f^{-1}\right]$ is finitely presented as a module over $B\left[f^{-1}\right]$,

then $1=0$ in $A$.

Proof. One can check that the claim is the interpretation by the sheaf semantics of the following statement about the sheaves: It is not not the case that

(1) $B^{\sim}$ and $M^{\sim}$ are free modules over $A^{\sim}$,

(2) $A^{\sim} \rightarrow B^{\sim}$ is of finite presentation, and

(3) $M^{\sim}$ is finitely presented as a module over $B^{\sim}$,

Item (1) is by basic linear algebra as in the proof of simple version of Grothendieck's generic freeness lemma, but a bit more involved as we now need to deal with countable generating families 80, Theorem 11.16]. Item (2) is because finitely generated algebras over anonymously Noetherian rings are not not finitely presented (in a description

${ }^{\mathrm{s}} \mathrm{A}$ standard result of linear algebra is: "Any finitely generated vector space over a field is finite free (that is, has a finite basis)." The proof proceeds by considering a given generating family and using the law of excluded middle to determine whether one of the generators can be expressed using the others or not. In the latter case, the family is linearly independent and hence a basis; in the former case, the proof removes the redundant generator and continues by induction.

Intuitionistically, we only have the double negation of the law of excluded middle available; this is why intuitionistically we only have that finitely generated vector spaces are not not finite free. The field condition required by the proof is exactly the one satisfied by $A^{\sim}$. 
of the form $R\left[X_{1}, \ldots, X_{n}\right] / \mathfrak{a}$, the ideal $\mathfrak{a}$ is not not finitely generated). Item (3) is because finitely generated modules over anonymously Noetherian are not not finitely presented.

A variant of this proof where the reference to the Noetherian condition has been replaced by an explicit computation has been unwound in Ref. 98 .

McCoy's theorem. It is a familiar fact from linear algebra that an injective $(n \times m)$-matrix $M$ over a geometric field has at least one invertible $m$-minor. Like many proofs in linear algebra, the proof proceeds by first applying elementary row and column transformations to put $M$ into the form $N:=\left(\begin{array}{cc}I & 0 \\ 0 & M^{\prime}\end{array}\right)$ where all entries in the submatrix $M^{\prime}$ are zero. This step exploits that any element is zero or invertible. Since $M$ is injective, the number of columns of $M^{\prime}$ is zero. Hence $N=\left(\begin{array}{l}I \\ 0\end{array}\right)$ has an invertible $m$ minor, and thus $M$ has well.

A version of McCoy's theorem states that this fact generalizes to arbitrary rings:

Theorem. Let $M \in A^{n \times m}$ be an injective matrix. Then $\left(\Lambda^{m} M\right)$, the ideal of $m$-minors of $M$, is regular.

The sheaf-theoretic reduction technique allows us to give a constructive proof of McCoy's theorem which is close to the linear algebra proof. In case that the ring $A$ is reduced, we can even copy the original proof almost word for word; for the general case, we require the following lemma.

Lemma. Let $M \in A^{n \times m}$ be an injective matrix with $m>0$. Assume that all entries of $M$ are nilpotent. Then $1=0$ in $A$.

Proof of the lemma. Let $(M)$ be the ideal generated by the entries of $M$. The assumption implies that there is a exponent $K \in \mathbb{N}$ such that $(M)^{K}=(0)$. If $K \geq 1$, then also $(M)^{K-1}=(0)$, since for $x \in$ $(M)^{K-1}$ the entries of $M(x, 0, \ldots, 0)^{t}$ are elements of $(M)^{K}$, hence zero, and $M$ is injective. We may thus assume $K=0$. So $1 \in(M)^{0}=(0)$.

Because we want to work in the generality of a ring which might not be reduced, we cannot use that $A^{\sim}$ is a field. Instead we will employ the generalization established in Exercise 34. Any nonunit of $A^{\sim}$ is nilpotent. The proof then proceeds as follows.

Proof of the theorem. Let $x \in A$ be such that $x\left(\Lambda^{m} M\right)=(0)$. We are to show $x=0$. By passing to the factor $\operatorname{ring} A / \operatorname{ann}(x)$, we may assume $\left(\Lambda^{m} M\right)=(0)$ and $x=1$; the new goal is to verify $1=0$. We do this by validating $\perp$ in the sheaf semantics. 
We may employ the law of excluded middle a finite number of times because we are to verify $\perp$. By repeatedly exploiting that any matrix entry is invertible or not, we may use row and column transformations to put $M$ into the form $N:=\left(\begin{array}{cc}I & 0 \\ 0 & M^{\prime}\end{array}\right)$ where all entries of $M^{\prime}$ are not invertible. Since nonunits of $A^{\sim}$ are nilpotent, the entries of $M^{\prime}$ are nilpotent, and since $M^{\prime}$ is injective and since $1 \neq 0$, the preceding lemma shows that the number of columns of $M^{\prime}$ is zero.

Hence $N=\left(\begin{array}{c}I \\ 0\end{array}\right)$ and $(1)=\left(\Lambda^{m} N\right)=\left(\Lambda^{m} M\right)=(0)$, a contradiction.

Remark 49. Not of pragmatic, but of logical interest is that the proof can also be recast as follows. Instead of reducing to the factor ring $A / \operatorname{ann}(x)$, we continue considering $A$. Working under the sheaf semantics, we then introduce a variant of the usual negation $\neg \varphi \equiv(\varphi \Rightarrow \perp)$, namely $\neg \varphi \equiv(\varphi \Rightarrow$ $x=0)$. We may still use the law of excluded middle for this new negation and hence exploit that any element $f$ of $A^{\sim}$ is invertible or "not" invertible. By Exercise 34, this amounts to saying that some power of $f$ annihilates $x$. The remainder of the proof carries over with minor modifications.

As with the other examples, the proof can mechanically be unwound to obtain a proof which is fully explicit:

Proof of the theorem. Let $x \in A$ be such that $x\left(\Lambda^{m} M\right)=(0)$. We are to show $x=0$. By passing to the factor $\operatorname{ring} A / \operatorname{ann}(x)$, we may assume $\left(\Lambda^{m} M\right)=(0)$ and $x=1$; the new goal is to verify $1=0$.

If $m=0$, the claim is immediate because in this case $(1)=\left(\Lambda^{m} M\right)=(0)$. So assume $m>0$.

Over the localized ring $A\left[M_{i j}^{-1}\right]$, the matrix entry $M_{i j}$ is invertible and hence $M$ can be put into the form $\left(\begin{array}{cc}1 & 0 \\ 0 & M^{\prime}\end{array}\right)$ by elementary row and column transformations. The matrix $M^{\prime}$ is still injective, and $\left(\Lambda^{m-1} M^{\prime}\right)=$ $\left(\Lambda^{m} M\right)$, hence we may assume by induction that $1=0$ in $A\left[M_{i j}^{-1}\right]$.

Hence all entries of $M$ are nilpotent and the claim follows from the preceding lemma.

Other short and explicit proofs of McCoy's theorem are given in Refs. 99], 14, Theorem 5.22] and [100, Exercise 5.23A(3)]. The proof in Ref. [101, Proposition III.8.2.3] employs the law of excluded middle. We were not able to discern that these proofs are just different presentations of the same idea that ours is based on; these proofs all employ computations with Cramer-like formulas, while our proof stresses the similarity to the field case.

It is on purpose that the (sheaf-theoretic version of) our proof contains case distinctions, to closely mimic the proof in the field case. It is also possible to do without, as already our unrolled proof demonstrates. Thierry 
Coquand and Claude Quitté gave a beautiful proof without case distinctions by applying a local-global principle [102, Theorem 2.4]; if desired, their proof can also be cast in the sheaf-theoretic framework (Exercise 35).

\section{Exercises}

Exercise 31 (Noetherian properties of the integers). (1)

Show that the law of excluded middle holds if the ring $\mathbb{Z}$ of integers is Noetherian in the sense that any ideal is finitely generated.

Hint. For a truth value $\varphi$, consider the ideal $\{x \in \mathbb{Z} \mid x=0 \vee \varphi\}$.

(2) Give an example of a space $X$ and a subsheaf $F$ of the constant sheaf $\underline{\mathbb{Z}}$ such that, under the sheaf semantics, the subsheaf $F$ is an ideal, and such that it is not the case that, again under the sheaf semantics, the ideal $F$ is finitely generated.

(3) Give a proof that $\mathbb{Z}$ is anonymously Noetherian which, unlike the proof given in Example 33, uses the anonymous version of the least number principle of Exercise 30

Exercise 32 (Free fields). (a) A free field over a set $M$ would be a field $K$ together with a map $M \rightarrow K$ such that any map $M \rightarrow L$ into a field $L$ factors over the given map $M \rightarrow K$ by a unique field homomorphism $K \rightarrow L$. Prove that free fields exist for no set $M$.

(b) Let $k$ be a field. Devise a notion of free $k$-fields (where a $k$-field is a field together with a homomorphism from $k$ ) and show that the only set $M$ such that there is a free $k$-field over $M$ is the empty set.

Exercise 33 (Nontriviality of $\boldsymbol{A}^{\sim}$ ). Let $A$ be a ring. In Proposition 37 , we gave an abstract proof that $A^{\sim}$ is (local and hence) nontrivial in the sense that $1 \neq 0$. This proof $\operatorname{did}$ not need to assume that $A$ itself is nontrivial. Give a new proof of this fact, by verifying directly $1 \models \neg\left(1={ }_{A^{\sim}}\right.$ $0)$ using the semantics given in Table 6 .

Exercise 34 (A generalization of the field property). Let $A$ be a ring. We extend the sheaf semantics to infinitary first-order formulas by adding the following clauses to Table 6

$$
\begin{aligned}
f \models \bigwedge_{k \in K} \varphi_{k} & \text { iff } \text { for all indices } k \in K, f \models \varphi_{k} \\
f \models \bigvee_{k \in K} \varphi_{k} & \text { iff } \\
& \text { there exists a partition } f^{n}=f g_{1}+\cdots+f g_{m} \text { such that, } \\
& \text { for each } i \text {, there is an index } k \in K \text { such that } f g_{i} \models \varphi_{k}
\end{aligned}
$$

Theorem 46 also applies to this extension, and moreover, this extension in sound with respect to infinitary intuitionistic logic. 
(a) Prove that:

$$
1 \models \forall f: A^{\sim} \cdot \forall x: A^{\sim} \cdot\left(\left(\exists z: A^{\sim} \cdot f z=1\right) \Rightarrow x=0\right) \Longrightarrow \bigvee_{n \in \mathbb{N}}\left(f^{n} x=0\right) .
$$

(b) Deduce that $A^{\sim}$ is "almost a field" in the sense that any nonunit is nilpotent.

(c) Show that $A$ is reduced iff $A^{\sim}$ is.

Exercise 35 (A local-global principle). Let $A$ be a ring. We introduce an operator $\nabla$ by

$$
\nabla \varphi: \equiv\left(\forall s: A^{\sim} \cdot((\varphi \Rightarrow s=0) \Rightarrow s=0) .\right.
$$

(a) Verify that $\nabla$ is a local operator, that is that

$$
(\varphi \Rightarrow \nabla \varphi), \quad(\nabla \nabla \varphi \Rightarrow \nabla \varphi) \quad \text { and } \quad((\nabla \varphi \wedge \nabla \psi) \Leftrightarrow \nabla(\varphi \wedge \psi))
$$

hold under the sheaf semantics.

(b) Show that equality is $\nabla$-stable in that

$$
1 \models \forall x: A^{\sim} \cdot \forall y: A^{\sim} \cdot \nabla(x=y) \Rightarrow x=y .
$$

Note. If $A$ is reduced, then $\nabla$ coincides with $\neg \neg$ and the claim reduces to statement F on page 47 In general, the operator $\nabla$ is related to the notion of scheme-theoretic dense open subsets [80, Lemma 9.11].

(c) Verify that an element of $A^{\sim}$ is regular iff it is $\nabla$-invertible, that is:

$$
1 \models \forall f: A^{\sim} .\left(\left(\forall s: A^{\sim} . f s=0 \Rightarrow s=0\right) \Longleftrightarrow \nabla\left(\exists g: A^{\sim} \cdot f g=1\right)\right)
$$

(d) Verify the analogous statement for finitely generated ideals:

$$
\begin{aligned}
& 1 \models \forall f_{1}: A^{\sim} \ldots \forall f_{n}: A^{\sim} . \\
& \quad\left(\left(\forall s: A^{\sim} .\left(\bigwedge_{i=1}^{n} f_{i} s=0\right) \Rightarrow s=0\right) \Longleftrightarrow \nabla\left(1 \in\left(f_{1}, \ldots, f_{n}\right)\right)\right)
\end{aligned}
$$

(e) Explain how the local-global principle "if $\left(f_{1}, \ldots, f_{n}\right)$ is a regular ideal and if an ideal $\left(g_{1}, \ldots, g_{m}\right)$ is regular when considered over each localization $A\left[f_{i}^{-1}\right]$, then $\left(g_{1}, \ldots, g_{m}\right)$ is regular over $A$ " can be viewed as an instance of modus ponens for local operators $((\nabla \varphi \wedge(\varphi \Rightarrow \nabla \psi)) \Longrightarrow \nabla \psi)$.

(f) Fill in the details, unwind the following proof of McCoy's theorem (page 66) and compare the result with the proof in Ref. [102, Theorem 2.4].

Theorem. Let $M \in A^{n \times m}$ be an injective matrix. Then $\left(\Lambda^{m} M\right)$, the ideal of $m$-minors of $M$, is regular.

Proof. We verify that, under $\nabla$, there exists an invertible $m$-minor, that is we verify $\nabla\left(\bigvee_{f}\left(\exists g: A^{\sim} . f g=1\right)\right)$, where the disjunction is over the $m$-minors of $M$. 
Since $M$ is injective, the ideal generated by the first column is regular, hence under $\nabla$ the unit ideal. Since our goal is $\nabla$-stable, we may assume that it actually is the unit ideal. Hence one of the entries in the first column is invertible. Applying elementary row and column transformations, we can put $M$ into the form $\left(\begin{array}{cc}1 & 0 \\ 0 & M^{\prime}\end{array}\right)$. Continuing in this fashion, we obtain the form $\left(\begin{array}{l}I \\ 0\end{array}\right)$. This matrix obviously has an invertible $m$-minor, and hence the original one has one as well.

\section{Exercise 36 (Bridging modules and their induced sheaves).}

Let $M$ be a module over a ring $A$.

(a) Show that $M$ is finitely generated iff $M^{\sim}$ is.

(b) Show that $M$ is finite locally free (that is, that there is a partition $1=$ $f_{1}+\cdots+f_{n}$ such that each module $M\left[f_{i}^{-1}\right]$ is finite free over $\left.A\left[f_{i}^{-1}\right]\right)$ iff $M^{\sim}$ is finite free.

(c) Show that $M$ is finitely presented iff $M^{\sim}$ is.

Exercise 37 (Local injectivity and surjectivity). Let $M$ and $N$ be modules over a ring $A$. Let $f: M \rightarrow N$ be a linear map.

(a) Show that $f$ is injective iff $f^{\sim}: M^{\sim} \rightarrow N^{\sim}$ is injective.

(b) Show that $f$ is surjective iff $f^{\sim}: M^{\sim} \rightarrow N^{\sim}$ is surjective.

Exercise 38 (Units of the sheaf model). Let $A$ be a ring. Let $f \in A$. Let $x \in A\left[f^{-1}\right]$. Show using Exercise 27 that $f \models \exists y: A^{\sim} . x y=1$ if and only if $x$ is invertible in $A\left[f^{-1}\right]$.

Exercise 39 (Decidability of invertibility). Show that it is not the case that any element of $\mathbb{Z}^{\sim}$ is invertible or not invertible.

Note. Since $\mathbb{Z}^{\sim}$ is a (sheaf) model of the theory of local rings, this observation shows that the theory of local rings does not prove that invertibility is decidable. Using much the same technique, this result has also been proven by Thierry Coquand 103 .

More generally, one can show: A ring $A$ is of Krull dimension $\leq n$ iff $A^{\sim}$ is; and a local ring (such as $A^{\sim}$ ) is of Krull dimension $\leq 0$ iff any element is invertible or nilpotent. Hence invertibility of elements of $A^{\sim}$ is decidable iff $A$ is of Krull dimension $\leq 0$. In this case, up to a technical condition, the universe of sheaves over $\operatorname{Spec}(A)$ is classical, hence any statement whatsoever is decidable. The ring controls the logic: Assuming that the metatheory is classical and assuming the technical condition, the universe of sheaves over $\operatorname{Spec}(A)$ is governed by classical logic iff $A^{\sim}$ is of Krull dimension $\leq 0$.

The for these purposes correct constructive notion of Krull dimension can be found in Refs. [104 105]. It has been used to give a short and constructive proof that $\operatorname{dim} k\left[X_{1}, \ldots, X_{n}\right]=n$, where $k$ is a geometric field [106. Proofs of the stated relation between the dimension of $A$ and the dimension of $A^{\sim}$ are recorded in Ref. 80, Section 3.4].

Exercise 40 (Triviality of the spectrum). Let $A$ be a ring. Verify, without assuming BPIT, that $\operatorname{Spec}(A)$ is isomorphic to the one-point locale iff any element of $A$ is either invertible or nilpotent. 
Hint. Use the description of the unique map $\mathcal{O}(\mathrm{pt}) \rightarrow \mathcal{O}(\operatorname{Spec}(A)), \varphi \mapsto \bigvee\{\top \mid \varphi\}$ computed in part (c) of Exercise 10

Exercise 41 (Locality of the spectrum). Let $A$ be a ring. Show that the spectrum of $A$ is local in the sense of Exercise 12 if and only if $A$ is local as a ring.

Exercise 42 (Spatiality of the spectrum). Let $A$ be a ring.

(a) Verify that $\operatorname{Spec}(A)$ is spatial if and only if for any element $x \in A$ and any ideal $\mathfrak{a} \subseteq A$,

$$
x \in \sqrt{\mathfrak{a}} \text { if } \text { for all prime filters } \mathfrak{f} \subseteq A \text { with } x \in \mathfrak{f}, \mathfrak{f} \varnothing \mathfrak{a},
$$

where the symbol " $\varnothing$ " denotes that the two sets have an element in common.

(b) Assuming the law of excluded middle, show that $\operatorname{Spec}(A)$ is spatial if and only if for any ideal $\mathfrak{a} \subseteq A$ the familiar identity

$$
\sqrt{\mathfrak{a}}=\bigcap_{\mathfrak{p} \supseteq \mathfrak{a}} \mathfrak{p}
$$

holds.

(c) Conclude that the spectrum of any ring is spatial iff BPIT holds.

Exercise 43 (Prüfer domains as valuation domains). An integral domain is a ring such that $1 \neq 0$ and such that $x y=0$ implies $x=0$ or $y=0$. A valuation domain is an integral domain such that for any two elements, one divides the other. A Prüfer domain is an integral domain such that any finitely generated ideal $\mathfrak{a}$ is locally a principal ideal (in the sense that there exists a partition $1=f_{1}+\cdots+f_{n}$ such that, for each index $i$, the ideal $\mathfrak{a}\left[f_{i}^{-1}\right]$ is a principal ideal in $\left.A\left[f_{i}^{-1}\right]\right)$.

(a) Let $A$ be a ring. Show that $A^{\sim}$ is an integral domain if $A$ is. Does the converse hold?

(b) Let $A$ be a valuation domain. Show that any matrix over $A$ can be put into diagonal form by elementary row and column operations.

(c) Let $A$ be an integral domain. Show that $A$ is a Prüfer domain if and only if $A^{\sim}$ is a valuation domain.

(d) Let $A$ be a Prüfer domain. Show that any matrix over $A$ can locally be put into diagonal form by elementary row and column operations, by applying the result of part (b) to $A^{\sim}$. 
Exercise 44 (A basic version of Kaplansky's theorem). (a) Let $A$ be a local ring. Let $\mathfrak{a} \subseteq A$ be a finitely generated ideal such that $\mathfrak{a}^{2}=\mathfrak{a}$. Show that $\mathfrak{a}=(0)$ or $\mathfrak{a}=(1)$.

Hint. Nakayama's lemma.

(b) Let $A$ be a local ring. Let $M \in A^{n \times n}$ be an idempotent matrix. Verify that $M$ is similar to a diagonal matrix with entries zero and one by applying part (a) to the ideals of $k$-minors of $M$. Deduce that the cokernel of $M$ is finite free.

(c) Let $A$ be an arbitrary ring. Let $M \in A^{n \times n}$ be an idempotent matrix. Show that the cokernel of $M$ is finite locally free, by applying the result of part (b) to $A^{\sim}$.

(d) Verify, without using the law of excluded middle or that $A$ is Noetherian, that an $A$-module $M$ is finitely generated and projective if and only if it is finite locally free.

Note. A self-contained solution is given in Ref. 107.

\section{Acknowledgments}

We are grateful to Peter Arndt, Andrej Bauer, Martin Brandenburg, Thierry Coquand, Martín Escardó, Simon Henry, Matthias Hutzler, Milly Maietti, Marc Nieper-Wißkirchen, Alexander Oldenziel, Anja Petković, Peter Schuster, Helmut Schwichtenberg, Steve Vickers and Daniel Wessel for invaluable discussions shaping this work. We are also grateful to the anonymous referee, whose comments suggested several improvements to the presentation.

We thank the organizers Klaus Mainzer, Peter Schuster and Helmut Schwichtenberg of Proof and Computation 2019, where this work was presented, for their kind invitation and for creating a beautiful and stimulating meeting. We also express our thanks to the local coordinator Chuangjie $\mathrm{Xu}$ and to all participants of that meeting.

\section{References}

[1] M. Maietti, Modular correspondence between dependent type theories and categories including pretopoi and topoi, Math. Structures Comp. Sci. 15 (6), 1089-1149 (2005).

[2] L. Crosilla. Set theory: constructive and intuitionistic ZF. In ed. E. Zalta, The Stanford Encyclopedia of Philosophy. Metaphysics Research Lab, Stanford University (2015). URL https://plato.stanford.edu/archives/ sum2015/entries/set-theory-constructive/. 
[3] P. T. Johnstone. The art of pointless thinking: a student's guide to the category of locales. In Category theory at work (Bremen, 1990), number 18 in Res. Exp. Math., pp. 85-107, Heldermann (1991).

[4] P. T. Johnstone, The point of pointless topology, Bull. Amer. Math. Soc. 8(1), 41-53 (1983).

[5] S. Vickers, Continuity and geometric logic, J. Appl. Log. 12(1), 14-27 (2014). URL https://www.cs.bham.ac.uk/ sjv/GeoAspects.pdf.

[6] S. Vickers, Locales and Toposes as Spaces, In eds. M. Aiello, I. PrattHartmann, and J. van Benthem, Handbook of Spatial Logics, pp. 429-496. Springer (2007). URL https://www.cs.bham.ac.uk/ sjv/LocTopSpaces. pdf.

[7] M. Bélanger and J.-P. Marquis, Menger and nöbeling on pointless topology, Logic Log. Philos. 22(2) (2013).

[8] J. Picado and A. Pultr, Frames and Locales. Topology without points. Front. Math., Birkhäuser (2012).

[9] D. Scott, Prime ideal theorems for rings, lattices, and boolean algebras, Bull. Amer. Math. Soc. 60(4), 390 (1954).

[10] B. Banaschewski and R. Harting, Lattice aspects of radical ideals and choice principles, Proc. Lond. Math. Soc. s3-50, 385-404 (1985).

[11] W. Savin. Minimal prime ideals and the axiom of choice (answer on mathoverflow). URL https://mathoverflow.net/a/98734 (2012).

[12] P. T. Johnstone, Sketches of an Elephant: A Topos Theory Compendium. Oxford University Press (2002).

[13] F. Richman, Nontrivial uses of trivial rings, Proc. Amer. Math. Soc. 103, 1012-1014 (1988).

[14] H. Lombardi and C. Quitté, Commutative Algebra: Constructive Methods. Springer (2015).

[15] T. Leinster, An informal introduction to topos theory, Publications of the nLab. 1(1) (2011).

[16] S. Mac Lane and I. Moerdijk, Sheaves in Geometry and Logic: a First Introduction to Topos Theory. Universitext, Springer (1992).

[17] G. Sambin, Some points in formal topology, Theoret. Comput. Sci. 305, 347-408 (2003).

[18] G. Sambin. Intuitionistic formal spaces - a first communication. In ed. D. Skordev, Mathematical Logic and its Applications, Proc. Adv. Internat. Summer School Conf., Druzhba, Bulgaria, 1986, pp. 187-204. Plenum (1987).

[19] P. Schuster, Formal Zariski topology: positivity and points, Ann. Pure Appl. Logic. 137(1), 317-359 (2006).

[20] T. Coquand, H. Lombardi, and P. Schuster, Spectral schemes as ringed lattices, Ann. Math. Artif. Intell. 56, 339-360 (2009).

[21] T. Coquand, H. Lombardi, and P. Schuster, The projective spectrum as a distributive lattice, Cah. Topol. Géom. Différ. Catég. 48(3), 220-228 (2007).

[22] M. Shulman. Stack semantics and the comparison of material and structural set theories. URL https://arxiv.org/abs/1004.3802 (2010). 
[23] A. Kock, Synthetic Differential Geometry, 2 edn. Number 333 in London Math Soc. Lecture Note Ser., Cambridge University Press (2006).

[24] J. M. E. Hyland. First steps in synthetic domain theory. In eds. A. Carbonia, M. Pedicchio, and G. Rosolini, Proc. of the International Conference held in Como, Italy, 1990, vol. 1488, Lecture Notes in Math., pp. 131-156, Springer (1991).

[25] A. Bauer. First steps in synthetic computability theory. In eds. M. Escardó, A. Jung, and M. Mislove, Proc. of the 21st Annual Conference on Mathematical Foundations of Programming Semantics, vol. 155, Electron. Notes Theor. Comput. Sci., pp. 5-31, Elsevier B.V. (2006).

[26] R. Mines, F. Richman, and W. Ruitenburg, A Course in Constructive Algebra. Universitext, Springer (1988).

[27] I. Yengui, Constructive Commutative Algebra. Projective Modules Over Polynomial Rings and Dynamical Gröbner Bases. Lecture Notes in Math., Springer (2015).

[28] M. Coste, H. Lombardi, and M.-F. Roy, Dynamical method in algebra: effective Nullstellensätze, Ann. Pure Appl. Logic. 111(3), 203-256 (2001).

[29] T. Coquand and H. Lombardi, A logical approach to abstract algebra, Math. Structures Comput. Sci. 16(5), 885-900 (2006).

[30] D. Rinaldi, P. Schuster, and D. Wessel, Eliminating disjunctions by disjunction elimination, Indag. Math. (N.S.). 29(1), 226-259 (2018). Virtual Special Issue - L.E.J. Brouwer, fifty years later.

[31] J. Cederquist and T. Coquand. Entailment relations and distributive lattices. In eds. S. Buss, P. Hájek, and P. Pudlák, Logic Colloquium '98. Proceedings of the Annual European Summer Meeting of the Association for Symbolic Logic, Prague, Czech Republic, August 9-15, 1998, vol. 13, Lect. Notes Log., pp. 127-139. A. K. Peters (2000).

[32] T. Coquand. A completeness proof for geometrical logic. In eds. P. Hájek, L. Valdés-Villanueva, and D. Westerståhl, Logic, Methodology and Philosophy of Science. Proceedings of the Twelfth International Congress, pp. 79-90, King's College Publications (2005).

[33] P. Aczel, The Russell-Prawitz modality, Math. Structures Comput. Sci. 11 (4), 541-554 (2001).

[34] M. Maietti, Joyal's arithmetic universes as list-arithmetic pretoposes, Theory Appl. Categ. 23(3), 39-83 (2010).

[35] S. Vickers. Sketches for arithmetic universes. URL https://arxiv.org/ abs/1608.01559 (2016).

[36] I. Blechschmidt and M. Hutzler. A constructive Knaster-Tarski proof of the uncountability of the reals. URL https://arxiv.org/abs/1902.07366 (2019).

[37] A. Joyal and M. Tierney, An Extension of the Galois Theory of Grothendieck. vol. 309, Mem. Amer. Math. Soc., American Mathematical Society (1984).

[38] A. Simpson, Measure, randomness and sublocales, Ann. Pure Appl. Logic. 163(11), 1642-1659 (2012).

[39] A. Bauer. König's lemma and the Kleene tree. URL http://math.andrej. 
com/wp-content/uploads/2006/05/kleene-tree.pdf (2006).

[40] J. Cederquist and S. Negri. A constructive proof of the Heine-Borel covering theorem for formal reals. In eds. S. Berardi and M. Coppo, Types for Proofs and Programs, pp. 62-75, Springer (1996).

[41] S. Vickers, Some constructive roads to Tychonoff, In eds. L. Crosilla and P. Schuster, From Sets and Types to Analysis and Topology: Towards Practicable Foundations for Constructive Mathematics, pp. 223-238. Oxford Univ. Press (2005).

[42] G. Wraith, Localic groups, Cah. Topol. Géom. Différ. Catég. 22, 61-66 (1981).

[43] G. Wraith, Galois theory in a topos, J. Pure Appl. Algebra. 19, 401-410 (1980).

[44] B. Banaschewski and C. Mulvey, A globalisation of the Gelfand duality theorem, Ann. Pure Appl. Logic. 137, 62-103 (2006).

[45] T. Coquand and B. Spitters, Constructive Gelfand duality for $C^{\star}$-algebras, Math. Proc. Cambridge Philos. Soc. 147 (2009).

[46] S. Henry. Constructive Gelfand duality for non-unital commutative $C^{\star}$ algebras. URL https://arxiv.org/abs/1412.2009 (2014).

[47] J. Butterfield, J. Hamilton, and C. Isham, A topos perspective on the kochen-specker theorem, i. quantum states as generalized valuations, Internat. J. Theoret. Phys. 37(11), 2669-2733 (1998).

[48] C. Heunen, N. Landsman, and B. Spitters, A topos for algebraic quantum theory, Comm. Math. Phys. 291(1), 63-110 (2009).

[49] S. Henry. A geometric bohr topos. URL https://arxiv.org/abs/1502. 01896 (2015).

[50] M. Fourman and D. Scott. Sheaves and logic. In eds. M. Fourman, C. Mulvey, and D. Scott, Applications of sheaves, vol. 753, Lecture Notes in Math., pp. 302-401, Springer (1979).

[51] J. Picado and A. Pultr, Locales mostly treated in a covariant way. vol. 41, Textos Mat. Sér. B, Universidade de Coimbra, Departamento de Matemática (2008).

[52] M. Rathjen. Remarks on Barr's theorem: proofs in geometric theories. In eds. D. Probst and P. Schuster, Concepts of Proof in Mathematics, Philosophy, and Computer Science, vol. 6, Ontos Math. Log., pp. 347-374. De Gruyter (2016).

[53] F. Lawvere, Quantifiers and sheaves, Actes Congrès Intern. Math. 1, 329334 (1970).

[54] M. Tierney. On the spectrum of a ringed topos. In eds. A. Heller and M. Tierney, Algebra, Topology, and Category Theory. A Collection of Papers in Honor of Samuel Eilenberg, pp. 189-210. Academic Press (1976).

[55] G. Wraith. Intuitionistic algebra: some recent developments in topos theory. In Proceedings of the International Congress of Mathematicians (1978, Helsinki), pp. 331-337, Acad. Sci. Fennica, Helsinki (1980).

[56] P. T. Johnstone, Topos Theory. vol. 10, L.M.S. Monographs, Academic Press (1977).

[57] S. Henry. Barr's theorem and constructivity? (question on MathOverflow). 
URL https://mathoverflow.net/questions/142217/ (2013).

[58] R. Goldblatt, Topoi: The Categorical Analysis of Logic. vol. 98, Stud. Logic Found. Math., Elsevier (1984).

[59] O. Caramello, Theories, Sites, Toposes: Relating and studying mathematical theories through topos-theoretic 'bridges'. Oxford University Press (2018).

[60] R. Dyckhoff and S. Negri, Geometrisation of first-order logic, Bull. Symbolic Logic. 21(2), 123-163 (2015).

[61] M. Rathjen and M. Toppel. On relating theories: Proof-theoretical reduction. In eds. S. Centrone, S. Negri, D. Sarikaya, and P. Schuster, Mathesis Universalis, Computability and Proof, vol. 412, Synthese Library, pp. 311331. Springer (2019).

[62] A. Tarizadeh. The flat topology and its duality aspects. URL https:// arxiv.org/abs/1503.04299v9 (2015).

[63] P. T. Johnstone, Rings, fields, and spectra, J. Algebra. 49(1), 238-260 (1977).

[64] I. Blechschmidt. Exploring mathematical objects from custom-tailored mathematical universes. In eds. G. Oliveri, S. Boscolo, and C. Ternullo, Philosophy of mathematics. Objects, Structures, and Logics (forthcoming). Springer (2020). URL https://rawgit.com/iblech/internal-methods/ master/paper-filmat.pdf.

[65] C. Mulvey. Intuitionistic algebra and representations of rings. In eds. K. H. Hofmann and J. R. Liukkonen, Recent Advances in the Representation Theory of Rings and $C^{*}$-algebras by Continuous Sections, vol. 148, Mem. Amer. Math. Soc., pp. 3-57, American Mathematical Society (1974).

[66] O. Caramello. Topos-theoretic background. URL https://www . oliviacaramello.com/Unification/ ToposTheoreticPreliminariesOliviaCaramello.pdf (2014).

[67] T. Streicher. Introduction to category theory and categorical logic. URL https://www.mathematik.tu-darmstadt.de/ streicher/CTCL.pdf (2004).

[68] F. Borceux, Handbook of Categorical Algebra: Volume 3, Sheaf Theory. Encyclopedia Math. Appl., Cambridge University Press (1994).

[69] M. Shulman. Categorical logic from a categorical point of view (draft for AARMS Summer School 2016). URL http://mikeshulman.github.io/ catlog/catlog.pdf (2016).

[70] M. Hyland. The effective topos. In eds. A. S. Troelstra and D. van Dalen, The L. E. J. Brouwer Centenary Symposium, pp. 165-216, North-Holland (1982).

[71] W. Phoa. An introduction to fibrations, topos theory, the effective topos and modest sets. Technical report, University of Edinburgh (1992). URL http://www.lfcs.inf.ed.ac.uk/reports/92/ ECS-LFCS-92-208/. ECS-LFCS-92-208.

[72] A. Bauer. Realizability as the connection between computable and constructive mathematics. URL http://math.andrej.com/asset/data/c2c. pdf (2005). 
[73] A. Ščedrov, Forcing and Classifying Topoi. vol. 295, Mem. Amer. Math. Soc., American Mathematical Society (1984).

[74] J. Hamkins and A. Lewis, Infinite time turing machines, J. Symbolic Logic. 65(2), 567-604 (2000).

[75] A. Bauer, An injection from the baire space to natural numbers, Math. Structures Comput. Sci. 25(7), 1484-1489 (2015).

[76] D. Normann and S. Sanders, On the mathematical and foundational significance of the uncountable, J. Math. Log. 18(2) (2018).

[77] F. Richman, The fundamental theorem of algebra: a constructive development without choice, Pac. J. Math. 196(1), 213-230 (2000).

[78] W. Ruitenburg. Constructing roots of polynomials over the complex numbers. In ed. A. Cohen, Computational Aspects of Lie Group Representations and Related Topics, Proc. of the 1990 Computer Algebra Seminar held in Amsterdam, vol. 84, CWI Tract, pp. 107-128, Centrum voor Wiskunde en Informatica, Amsterdam (1991).

[79] D. Bridges, F. Richman, and P. Schuster, A weak countable choice principle, Proc. Amer. Math. Soc. 128(9) (2000).

[80] I. Blechschmidt. Using the internal language of toposes in algebraic geometry. PhD thesis, University of Augsburg (2017). URL https://rawgit. com/iblech/internal-methods/master/notes.pdf.

[81] F. Richman, The ascending tree condition: constructive algebra without choice, Comm. Algebra. 31(4), 1993-2002 (2003).

[82] H. Perdry, Strongly noetherian rings and constructive ideal theory, J. Symbolic Comput. 37(4), 511-535 (2004).

[83] H. Perdry, Lazy bases: a minimalist constructive theory of Noetherian rings, MLQ Math. Log. Q. 54(1), 70-82 (2008).

[84] H. Perdry and P. Schuster, Noetherian orders, Math. Structures in Comput. Sci. 21(1), 111-124 (2011).

[85] J. Tennenbaum. A constructive version of Hilbert's basis theorem. PhD thesis, University of California (1973).

[86] N. Kraus, M. Escardó, T. Coquand, and T. Altenkirch, Notions of anonymous existence in Martin-Löf type theory, Log. Methods Comput. Sci. 13 (1) (2017).

[87] M. Atiyah and I. Macdonald, Introduction to Commutative Algebra. Addison-Wesley (1969).

[88] E. Palmgren and S. Vickers, Partial horn logic and cartesian categories, Ann. Pure Appl. Logic. 145(3), 314-353 (2007).

[89] M. Coste. Localisation, spectra and sheaf representation. In eds. M. Fourman, C. Mulvey, and D. Scott, Applications of sheaves, vol. 753, Lecture Notes in Math., pp. 212-238, Springer (1979).

[90] J. Cole, The bicategory of topoi and spectra, Repr. Theory Appl. Categ. 25, 1-16 (2016).

[91] L. Crosilla, Exploring predicativity, In eds. K. Mainzer, P. Schuster, and H. Schwichtenberg, Proof and Computation, pp. 83-108. World Scientific (2018). 
[92] P. Aczel and M. Rathjen. Constructive set theory (book draft). URL https://www1.maths.leeds.ac.uk/ rathjen/book.pdf (2010).

[93] J. Dieudonné and A. Grothendieck, Éléments de géométrie algébrique: IV. Étude locale des schémas et des morphismes de schémas, Seconde partie, Publ. Math. Inst. Hautes Études Sci. 24 (1965).

[94] H. Matsumura, Commutative Ring Theory. vol. 8, Cambridge Stud. Adv. Math., Cambridge University Press (1987).

[95] D. Eisenbud, Commutative Algebra with a View Toward Algebraic Geometry. vol. 150, Grad. Texts in Math., Springer (1995).

[96] C. Staats. Elementary proof of generic freeness. URL https://math.uchicago.edu/ cstaats/Charles_Staats_III/ Notes_and_papers_files/generic_freeness.pdf (2011).

[97] The Stacks Project Authors. Stacks Project. URL https://stacks .math. columbia.edu/.

[98] I. Blechschmidt. An elementary and constructive proof of Grothendieck's generic freeness lemma. URL https://arxiv.org/abs/1807.01231 (2018).

[99] Z. Błocki, An elementary proof of the McCoy theorem, Univ. Iagel. Acta Math. 30, 215-218 (1993).

[100] T. Lam, Exercises in Modules and Rings. Problem Books in Math., Springer (2007).

[101] N. Bourbaki, Algebra I, Chapters 1-3. Addison-Wesley (1973).

[102] T. Coquand and C. Quitté, Constructive finite free resolutions, Manuscripta Math. 137, 331-345 (2012).

[103] T. Coquand. A remark about the theory of local rings. URL http://www . cse.chalmers.se/ coquand/local.pdf (2008).

[104] T. Coquand, L. Ducos, H. Lombardi, and C. Quitté, Constructive Krull dimension I: integral extensions, J. Algebra Appl. 8(1), 129-138 (2009).

[105] T. Coquand, H. Lombardi, and M.-F. Roy, An elementary characterisation of Krull dimension, In From Sets and Types to Analysis and Topology: Towards Practicable Foundations for Constructive Mathematics, pp. 239244. Oxford Univ. Press (2005).

[106] T. Coquand and H. Lombardi, A short proof for the Krull dimension of a polynomial ring, Amer. Math. Monthly. 112(9), 826-829 (2005).

[107] I. Blechschmidt. Vector bundles on affine schemes (short note). URL https://www.ingo-blechschmidt.eu/kaplansky-en.pdf (2015). 